\title{
A Lifetime Measurement of the Exclusive Charged and Neutral B Meson States
}

\author{
by \\ J. D. Cammerata \\ A dissertation submitted to \\ The Johns Hopkins University \\ in conformity with the requirements for the \\ degree of Doctor of Philosophy \\ Baltimore, Maryland. \\ 1996 \\ (C) by Jeffrey Donald Cammerata 1996 \\ All rights reserved
}




\begin{abstract}
This thesis reports a measurement of the charged and neutral $B$ lifetimes using the decay modes $\mathrm{B} \rightarrow \Psi \mathrm{K}$ where $\mathrm{B}$ symbolizes $B_{u}$ or $B_{d}, \Psi$ denotes $\mathrm{J} / \psi$ or $\psi(2 \mathrm{~S})$, and $\mathbf{K}$ denotes $\mathrm{K}, K_{s}^{0}$, or $K^{*}(892)$. The $B_{u}$ lifetime is $1.68 \pm$ 0.07 (stat) \pm 0.02 (sys)ps, the $B_{d}$ lifetime of $1.58 \pm 0.09$ (stat) \pm 0.02 (sys) ps, and the charged to neutral lifetime ratio is $1.06 \pm 0.07$ (stat) \pm 0.01 (sys). A $B_{s}^{0}$ lifetime is presented for the decay modes $B_{s}^{0} \rightarrow \mathrm{J} / \psi \phi, \psi(2 \mathrm{~S}) \phi$ as a consistency check. The supervising professor responsible for the support of this analysis is Dr. Bruce Barnett.
\end{abstract}




\section{Acknowledgements}

Of course, no project of this size is completed without the help of others in the academic sense. I wish to thank Dr. Barnett for 'taking me under his wing' throughout my graduate career and, particularly, during the first couple of years. He demonstrates faith in his students to complete tasks, by giving them the freedom to chose their own path to completion. I valued this above any academic contributions he made during my tour of duty. In following that thought, I wish to thank the professors, administration, and coaches from football and volleyball at Franklin and Marshall college for the well rounded interests that I currently enjoy.

Thanks to Paul Ratzmann, Mike Lindenmeyer, Mike Hrycyk, Chuck Grimm, Bob Shovan, and the the rest of the techs and engineers with whom I worked on SVX II. I would like to give special thanks to four people, Estella Hankin, Jack Spangler, Joe Orndorff, and Dave Newman. These friends supported my academic endeavors during my years in Baltimore and helped me grow as a human being which is ultimately more important than any academic knowledge I could ever obtain. Also, thanks to my JHU volleyball teammates for our undefeated season in ' 92 .

I wish to express my appreciation on behalf of Dr. Boyer who was my last physics instructor at my high school. His approach to students as colleagues allowed us to learn efficiently, by expecting a maturity level that, otherwise, would have remained dormant. Finally, special thanks go to my extended family, and most notably, my parents. In the Italian tradition, one could not ask for more support than I received from the both of them.

This thesis is dedicated to Pap Magistro who passed away in the Spring of ' 96 .

J. D. Cammerata

July 10, 1996 


\section{Contents}

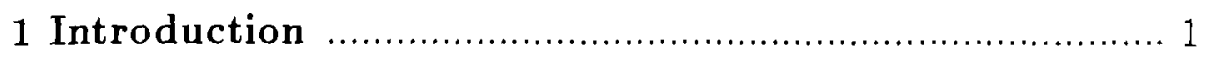

2 Theoretical Background ……...................................... 4

2.1 A Particle History …................................................. 4

2.2 The Current State of Affairs ........................................ 6

2.2.1 Fundamental Particles ....................................... 6

2.2.2 Electroweak Interaction and

the Cabibbo-Kobayashi-Maskawa Matrix .................... 8

2.2.3 Heavy Quark Effective Theory ......................... 13

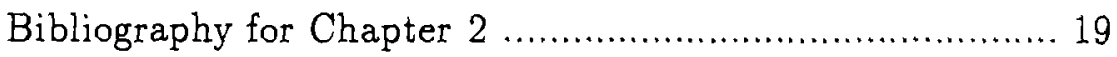

3 Hardware and Software ……....................................... 22

3.1 The Accelerator ….................................................. 22

3.2 The Detector …................................................... 30

3.2.1 Muon Chambers ........................................ 30

3.2.2 Central Tracking Chamber ............................... 33

3.2.3 The Silicon Vertex Detector .............................. 36 
3.2.4 Trigger Levels .................................................. 39

3.2.5 Data Sets and Software Analysis ....................... 42

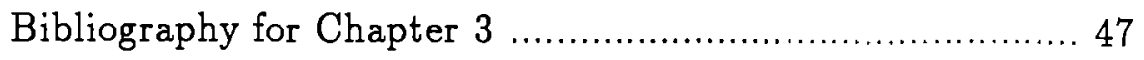

4 B Meson Reconstruction ….......................................... 49

4.1 Decay Products ......................................................... 49

4.1.1 Dimuon Candidate Selection ............................... 49

$4.1 .2 \Psi$ and $\mathrm{K}_{\mathrm{g}}^{\mathrm{o}}$ Reconstructions ................................ 53

$4.1 .3 \mathrm{~B}_{u}, \mathrm{~B}_{d}$, and $\mathrm{B}_{\boldsymbol{s}}$ Mesons ....................................... 58

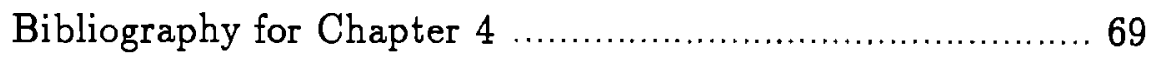

5 Lifetime Analysis …................................................... 70

5.1 Calculation of $\mathrm{c} \tau$..................................................... 70

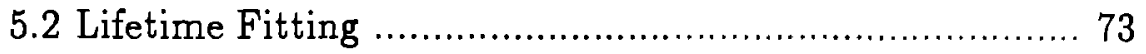

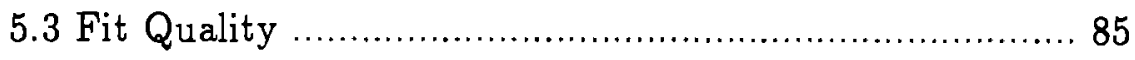

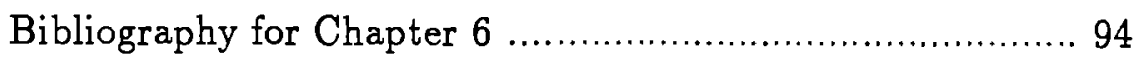

6 Systematic Error Analysis ……................................. 95

6.1 Data Sample Systematics .......................................... 95

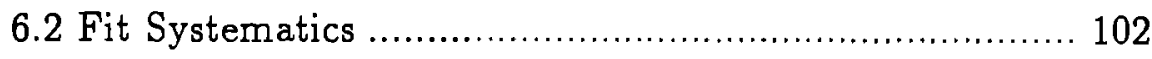



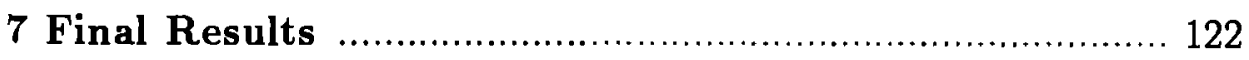

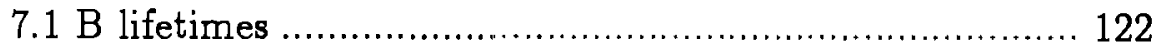






\section{Chapter 1}

\section{Introduction}

One of the fundamental reasons for studying high energy physics is to determine the origin of matter. Darwin's The Origin of Species sought the answer to essentially the same question. High energy physics has the distinct advantage of being able to run the movie backwards. The way this is done is to generate in a very small place, for a very short period of time, the same ambient energy that existed shortly after the first instant of time. By doing this one learns the hierarchy of large to small by observing the creation and decay processes that exist at such a high energy. This hierarchy extends from quasars to galaxies, from stars to planets, from molecules to atoms, and from nucleii to quarks. Two shared themes among biological organisms and high energy particles are life and death. Just as organisms live and die according to the laws of biochemistry, high energy particles live and die according to the laws of physics. In the most general sense this paper is a biography. It tells the story of the lives of two particles: how they were born, how they lived, and how they died.

The analysis presented here stems from data taken at Fermilab National Accelerator located in Batavia, Il. Data was taken over several years with a break to upgrade various detector and DAQ systems. The first and second parts of the data sample are referred to as Run $1 \mathrm{~A}$ and Run $1 \mathrm{~B}$ in which 
1B started after the upgrade. The events of interest contain highly energetic remnants from collisions between protons and antiprotons. Many of the particles created from the ambient energy available from the collisions are highly unstable and decay directly or via a decay chain to particles that exist in ordinary matter. Typically, the heavier the particle produced the faster it decays and the longer the decay chain until only stable particles remain. Some of the heavier particles seen at Fermilab involve b quarks. To date, the $b$ quark is the second heaviest among the six quarks know to exist. Quarks cannot be observed directly but their evidence can be seen through their interactions with other quarks. The type of decay chain reveals the structure of the parent particle. This paper discusses several different decay chains that trace their lineage to a quark-antiquark pair that contain a single b. Quark-antiquark bound states with a single b quark are referred to as $\mathrm{B}$ mesons. These mesons travel and decay over a definite time interval and decay leaving signature daughter products in their wake. This analysis specifically looks for these daughter products, finds their common point of origin, and obtains a flight path for the parent $B$. With knowledge of the flight path and the momentum, the lifetime of the $B$ is calculated over a sizable statistical sample.

Alan Spies and Olivier Snider initiated this analysis for Run 1A. Since this paper is an extension of Run $1 \mathrm{~A}$, obvious and unavoidable similarities exist in Run 1B. First and foremost is content. The same decay channels for the $B_{d}$ and the $B_{u}$ are used. The $B_{u}$, or $B^{ \pm}$, mesons are reconstructed from 4 decay modes:

- $\mathrm{J} / \psi K^{ \pm}$

- $\mathrm{J} / \psi K^{*}(892)^{ \pm}$

- $\Psi(2 S) K^{ \pm}$

- $\Psi(2 S) K^{*}(892)^{ \pm}$ 
The neutral B mesons, $B_{d}$ or $B^{\circ}$, are also reconstructed from 4 decays:

- $\mathrm{J} / \psi K^{*}(892)^{\circ}$

- $\mathrm{J} / \psi K_{s}^{\circ}$

- $\Psi(2 S) K^{*}(892)^{\circ}$

- $\Psi(2 S) K_{s}^{\circ}$

Of course the conjugate is included in the above decays to include $\overline{B^{\circ}}$. The reconstruction of the daughter products such as $K^{*}(892)^{\circ}$ follows a similar approach to $1 \mathrm{~A}$. Other major features of the $1 \mathrm{~A}$ analysis were kept basically in tact: momentum, mass, and track quality cuts, duplicate event removal, and fitting procedures.

However, while the content is similar the approach to $1 \mathrm{~B}$ is distinctly different from 1A. One of the major differences is in experimental apparatus. The Silicon Vertex Detector from Run 1A was replaced in 1993 with an improved version for Run 1B. Software changes accompanied equipment changes. Because of the existence of a new SVX the geometry database was updated. This new geometry and upgraded offline packages were implemented for processing the 1B data. Run 1A data was also reprocessed using the new offline packages with the correct geometrical database. The code responsible for the lifetime analysis was modified in order to be compatible with the location and content of the new versions of the offline packages. The final major difference between Run $1 \mathrm{~A}$ and $1 \mathrm{~B}$ is the amount of data available for analysis. The $1 \mathrm{~A}$ lifetime result was obtained using $20 \mathrm{pb}^{-1}$ of data. My analysis combines Run $1 \mathrm{~A}$ data with nearly $90 \mathrm{pb}^{-1}$ of data from Run 1B to complete the measurement for Run I. Data from Run 1B is used from the Fall of 1993 to the Summer of 1995. A small series of runs in the Winter of 1996 was used primarily for Tevatron studies and was deemed not useful for inclusion in this analysis. 


\section{Chapter 2}

\section{Theoretical Background}

In any field the accuracy of prediction is predicated on a sound fundamental theory. Currently, the Standard Model in the field of high energy physics has the flexibility to account for the large numbers of particles seen at particle colliders.

\subsection{A Particle History}

Before the mid 1930's the proton, neutron, and electron were considered fundamental particles. Cosmic ray experiments from the mid 1930's through the 1950 's produced scores more particles complicating the picture. Particles such as the pion and muon had masses between that of the proton and electron and interacted differently with matter than expected. The existence of a particle of intermediate mass was predicted by Yukawa to account for the nuclear forces. The range of the nuclear force is about $1 \mathrm{fm}$. For a virtual particle to exist that is responsible for the force that binds nucleons together, its travel time is

$$
t=\frac{\hbar}{m c^{2}}
$$


over a distance,

$$
c t=\frac{\hbar c}{m c^{2}}=200 \mathrm{MeV} \cdot \mathrm{fm} / \mathrm{mc}^{2}
$$

given by the uncertainty principle. This formula leads to a mass of about 200 $\mathrm{MeV} / \mathrm{c}^{2}$ for a range of $1 \mathrm{fm}$. Because of the small muon-pion mass difference and the large uncertainties on their measured masses, muons were confused for charged pions. Not until the observation of $\pi^{+} \rightarrow \mu^{+} \nu_{\mu}$ and $\pi^{-} \rightarrow \mu^{-} \bar{\nu}_{\mu}$ in photographic emulsions[2.1] was the ambiguity removed. The confirmation of $\pi^{\circ} \rightarrow \gamma \gamma$ at Berkeley[2.2], completed the pion charge spectrum and brought elementary particle physics to a temporary close, although the only particle not predicted by theory was the muon.

The discovery of even more new particles demanded the removal of certain prejudices in the field. One main prejudice was the belief that parity was conserved in all interactions and decays. The $\theta-\tau[2.3]$ puzzle precipitated a rethinking of that belief. The $\tau$ meson was observed to decay to $\pi^{+} \pi^{+} \pi^{-}[2.4]$ while the $\theta$ went to $\pi^{+} \pi^{0}$. Both had very similar masses but were considered different particles because of the different spin-parity of the decay states. To consider them to be the same particle was to violate parity. Nakano and Nishijima and independently Gell-Mann[2.5] proposed a new classification scheme assigning particle states on the basis of three quantum parameters to justify the notion that the $\theta$ and $\tau$ were the same particle. Particles were organized according to strangeness $\mathrm{S}$, isospin $\mathrm{I}$, and baryon number $\mathrm{B}$ by the famous Gell-Mann-Nishijima formula,

$$
Q=I_{3}+(B+S) / 2
$$

where $I_{3}$ is the $\mathrm{z}$ projection of the isospin vector and $\mathrm{Q}$ is the particle charge. Strong interactions conserved strangeness and isospin while weak interactions violated isospin conservation and changed strangeness by one unit. This explained the phenomena of associated production. Reactions like

$$
\pi^{-} p \rightarrow n+K^{+}+K^{-}
$$


involve the strong interaction, so that the total $S$ on both sides is equal to zero. The charged K's then decay via the weak interaction and $\Delta S= \pm 1$ for $K^{ \pm}$decays. With the discovery of new baryon and meson resonances, pieces of the particle puzzle began to fall into place. The last two pieces completing the SU(3) symmetry were the $\eta$ discovered by Pevsner and Block[2.6] and the $\Omega^{-}$found by Samios and Shutt[2.7]. The $\eta$ and the $\Omega^{-}$were the last members the famous pseudoscalar meson octet and $J^{P}=3 / 2^{+}$baryon decuplet, respectively.

The current state of particle physics is in some ways much like that before the discovery of the $\eta$ and $\Omega$. According to the Standard Model most of the mainstream pieces currently believed to exist have been discovered with the exception of the Higgs. The top quark was the latest piece to be discovered at $\mathrm{CDF}$ [2.8]. It is appropriate here to describe the current state of particle physics and see where important features of it are germane to the exclusive $\mathrm{B}$ lifetime analysis.

\subsection{The Current State of Affairs}

\subsubsection{Fundamental Particles}

One way to classify particles is according to the type of interaction in which it participates. Four fundamental forces govern the behavior of all matter. The four fundamental forces and their respective force carrying mediators are given in Table 2.1 .

\begin{tabular}{|l|l|}
\hline Force & Gauge Particle \\
\hline Gravitation & graviton? \\
Electromagnetism & photon \\
Strong Nuclear Interaction & gluon \\
Weak Nuclear Interaction & weak bosons \\
\hline
\end{tabular}

Table 2.1: The fundamental forces. 
The force of gravity and electromagnetism are infinite in range, while the range of the strong and weak nuclear forces are confined to the infinitesimal dimensions of the nucleus. Particles that feel the strong nuclear force are called hadrons. Hadrons are not fundamental particles but composed of smaller constituents called quarks. Baryons are hadrons containing three quarks $(q q q)$ and mesons are composed of quark-antiquark pairs $(q \bar{q})$. A total of six quarks and their corresponding antiquarks comprise all hadronic matter seen at particle colliders. Quarks are arranged in three generations given by Table 2.1

$$
\left(\begin{array}{l}
u \\
d
\end{array}\right)\left(\begin{array}{l}
c \\
s
\end{array}\right)\left(\begin{array}{l}
t \\
b
\end{array}\right)
$$

Table 2.1: From left to right the first, second, and third generation quarks according to the Standard Model.

The first generation quarks are roughly one third the proton mass. In contrast to the similar masses of the first generation, the third generation quarks differ dramatically in mass as the top mass is almost $170 \mathrm{GeV} / \mathrm{c}^{2}$ greater than the $b$ mass. The charge of the quark bound states are determined by

$$
Q=I_{3}+(B+S+C+B+T) / 2
$$

which is the full form of the Gell-Mann-Nishijima relation. The additional quantum numbers are a direct result of the flavor of the quark. For example, $\mathrm{C}$, represents the charm content of a hadron, B, represents the beauty content, and, T, represents the top content. Quantum Chromodynamics is the branch of theory that describes how gluons react with quarks and other gluons. Responsible for binding quarks together, gluons are described as vector gauge bosons that are members of an $\mathrm{SU}(3)$ non-Abelian group. The fact that they are gauge particles implies that the associated color symmetry is local, unlike the global symmetry resulting from the $\mathrm{SU}(3)$ flavor group. 
QCD is a fully relativistic theory rooted in QED in which iterative perturbations are based on increasing orders of magnitude of a coupling constant. Since the QED coupling constant, $\alpha_{Q E D}$, is significantly less than 1 and the strong coupling constant, $\alpha_{Q C D}$, is on the order of 1 , this perturbative approach is less effective for QCD than QED. Section 2.3 describes an effective theory in which the perturbative expansion is something other than the strong coupling constant and does a very adequate job in describing quark bound states above a certain mass threshold.

Indifference to the strong interaction qualifies the particle as a lepton, from the Greek leptos meaning small, fine, or light in weight. Leptons and the corresponding antileptons are considered fundamental and are arranged in three generations similar to those of the quarks. The leptons are shown in Table 2.2 .

$$
\left(\begin{array}{c}
\nu_{e} \\
e
\end{array}\right)\left(\begin{array}{c}
\nu_{\mu} \\
\mu
\end{array}\right)\left(\begin{array}{c}
\nu_{\tau} \\
\tau
\end{array}\right)
$$

Table 2.2 The 3 Generations of leptons according to the Standard Model.

The $\mu$ and $\tau$ leptons are heavier, short lived relatives of the electron in which the muon is about 200 times the mass of the electron and the $\tau$ is close to $4000 m_{e}$. Each particle has a corresponding massless antineutrino that is listed as the lower member in each family of Table 2.2 .

\subsubsection{The Electroweak Interaction and the Cabibbo- Kobayashi-Maskawa Matrix}

An $\mathrm{SU}(2)_{L}$ gauge group[2.9] represents the bosons of the weak nuclear force while the photon is simply represented by $\mathrm{U}(1)$. The symmetries associated with $\mathrm{SU}(2)_{L}$ and $\mathrm{U}(1)$ groups give rise to the quantum numbers of weak isospin and weak hypercharge, respectively. Glashow[2.10], Salam[2.11], and Weinberg[2.12] unified electromagnetism and the weak nuclear force into one 
electroweak force described by an $\mathrm{SU}(2)_{L} \times \mathrm{U}(1)$ gauge group. All fundamental fermions participate in the electroweak interaction giving rise to an extended field theory of Quantum Flavor Dynamics. Weak hypercharge and weak isospin are related to the charge matrix of the fundamental fermions by

$$
\mathbf{Q}=\mathbf{T}_{3}+\mathbf{Y}
$$

The values of weak isospin and hypercharge are given in Tables 2.3 and 2.4 for both quarks and leptons. The left handed components form isodoublets in contrast to the isosinglet states of the right handed components. The hypercharge values are hand picked to agree with the observed charges of the various particles. As in QED, a set Feynman graphs for the Standard Electroweak Model exist and are shown in Figure 2.1 for the tree graphs in the unitary gauge. The tenet of charged and neutral current vertex factors are listed in Tables 2.5 and 2.6 to include quarks and leptons. Quark color and flavor are unchanged with the emission (absorption) of a photon or $\mathrm{Z}$ boson, while the emission of a $\mathrm{W}$ boson leads to a change in flavor leaving the quark color the same. For completeness gluon emission implies a change in quark color but not flavor.

\begin{tabular}{|c|c|c|c|c|c|c|}
\hline \multicolumn{3}{|c|}{ Leptons } & $\mathrm{T}$ & $T_{3}$ & $\mathrm{Y}$ & $\mathrm{Q}$ \\
\hline$\nu_{e_{L}}$ & $\nu_{\mu_{L}}$ & $\nu_{\tau_{L}}$ & $\frac{1}{2}$ & $\frac{1}{2}$ & $-\frac{1}{2}$ & 0 \\
$e_{L}$ & $\mu_{L}$ & $\tau_{L}$ & $\frac{1}{2}$ & $-\frac{1}{2}$ & $-\frac{1}{2}$ & -1 \\
\hline$e_{R}$ & $\mu_{R}$ & $\tau_{R}$ & 0 & 0 & -1 & -1 \\
\hline
\end{tabular}

Table 2.3: Weak isospin and hypercharge values of the isosinglet and isodoublet lepton states. 


\begin{tabular}{|c|c|c|c|c|c|c|}
\hline \multicolumn{3}{|c|}{ Quarks } & $\mathrm{T}$ & $T_{3}$ & $Y$ & $\mathrm{Q}$ \\
\hline$u_{L}$ & $c_{L}$ & $t_{L}$ & $\frac{1}{2}$ & $\frac{1}{2}$ & $\frac{1}{6}$ & $\frac{2}{3}$ \\
\hline$d_{L}^{\prime}$ & $s_{L}^{\prime}$ & $b_{L}^{\prime}$ & $\frac{1}{2}$ & $-\frac{1}{2}$ & $\frac{1}{6}$ & $-\frac{1}{3}$ \\
\hline$u_{R}$ & $c_{R}$ & $t_{R}$ & 0 & 0 & $\frac{2}{3}$ & $\frac{2}{3}$ \\
\hline$d_{R}$ & $s_{R}$ & $b_{R}$ & 0 & 0 & $-\frac{1}{3}$ & $-\frac{1}{3}$ \\
\hline
\end{tabular}

Table 2.4: Weak isospin and hypercharge values of the isosinglet and isodoublet quark states. The d', s', and b' states are the result of a generalized Cabibbo rotation of the $d, s$, and b quark states.
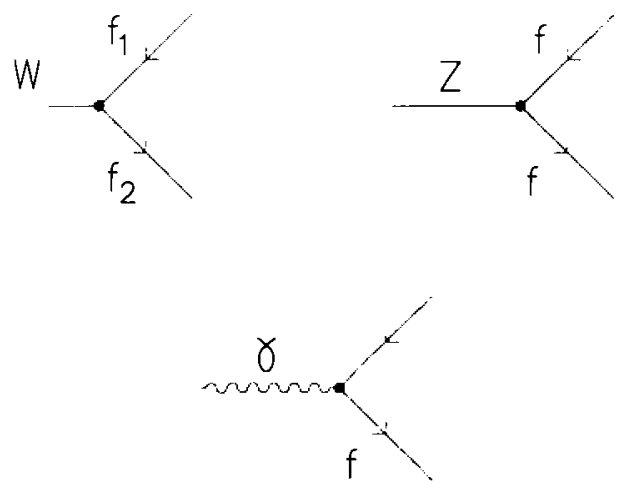

Figure 2.1: First order couplings in the Standard Electroweak Model. 


\begin{tabular}{|c|c|c|}
\hline$f_{1}$ & $f_{2}$ & Vertex Factor \\
\hline$\nu_{l}$ & $l^{-}$ & $-\frac{i g}{\sqrt{2}} \gamma_{\mu} \frac{1-\gamma_{5}}{2}$ \\
\hline$q_{1}$ & $q_{2}$ & $-\frac{i g}{\sqrt{2}} V_{q_{1} q_{2}} \gamma_{\mu} \frac{1-\gamma_{b}}{2}$ \\
\hline
\end{tabular}

Tables 2.5 and 2.6: (Above) Charged current vertex factors. (Below) Neutral current vertex factor expressed in terms of the vector and axial coupling constants with corresponding values listed for each fermion class.

\begin{tabular}{|c|l|l|l|c|}
\hline \hline $\mathrm{f}$ & $C_{L}$ & $C_{R}$ & $g_{V}=C_{L}+C_{R}$ & $g_{A}=C_{L}-C_{R}$ \\
\hline$\nu$ & $\frac{1}{2}$ & 0 & $\frac{1}{2}$ & $\frac{1}{2}$ \\
$l^{-}$ & $-\frac{1}{2}+\sin ^{2} \theta_{W}$ & $\sin ^{2} \theta_{W}$ & $-\frac{1}{2}+\sin ^{2} \theta_{W}$ & $-\frac{1}{2}$ \\
$q_{1}\left(\mathrm{Q}=\frac{2}{3}\right)$ & $\frac{1}{2}-\frac{2}{3} \sin ^{2} \theta_{W}$ & $-\frac{2}{3} \sin ^{2} \theta_{W}$ & $\frac{1}{2}-\frac{4}{3} \sin ^{2} \theta_{W}$ & $\frac{1}{2}$ \\
$q_{2}\left(\mathrm{Q}=-\frac{1}{3}\right)$ & $-\frac{1}{2}+\frac{1}{3} \sin ^{2} \theta_{W}$ & $\frac{1}{3} \sin ^{2} \theta_{W}$ & $-\frac{1}{2}+\frac{2}{3} \sin ^{2} \theta_{W}$ & $-\frac{1}{2}$ \\
\hline
\end{tabular}

The $\mathrm{W}$ and $\mathrm{Z}[2.13,2.14]$ were discovered first at CERN with masses of 83 and $92 \mathrm{GeV} / \mathrm{c}^{2}$, respectively. The $\mathrm{SU}(2)_{L} \times \mathrm{U}(1)$ symmetry prohibits the $\mathrm{W}$ and $\mathrm{Z}$ from being massive, therefore the symmetry must be manifestly broken. Putative theory explains that the spontaneously broken symmetry is equivalent to chosing a specific degenerate state and leads to the masses of the three vector bosons by introducing a Higgs mechanism[2.15]. In the 
minimal standard model the Higgs field is chosen as an isodoublet,

$$
\phi(x)=\left(\begin{array}{l}
\phi^{\dagger}(x) \\
\phi^{\circ}(x)
\end{array}\right)
$$

where $\phi^{\dagger}(x)$ and $\phi^{\circ}(x)$ are complex scalar fields. A pedantic derivation of the full $\mathrm{SU}(2)_{L} \times \mathrm{U}(1)$ Lagrangian reveals that the intermediate vector bosons (IVB) masses are a function of Higgs parameters:

$$
\begin{gathered}
M_{W}=g v / 2 \\
M_{Z}=\left(g^{2}+g^{\prime 2}\right)^{1 / 2} v / 2
\end{gathered}
$$

leading to,

$$
M_{W}=M_{Z} \cos \theta_{W}
$$

The relationship between the original V - A theory of $\beta$ decay and the parameters in the standard electroweak model are given by:

$$
\begin{gathered}
G_{F} / \sqrt{2}=\frac{g^{2}}{8 M_{W}^{2}} \\
g \sin \theta_{W}=g^{\prime} \cos \theta_{W}=e .
\end{gathered}
$$

A discussion of the CKM matrix completes the major features of the relativistic theory of the Standard Model. Assuming 3 quark generations the most general form of the matrix is given by

$$
V=\left[\begin{array}{lll}
V_{u d} & V_{u s} & V_{u b} \\
V_{c d} & V_{c s} & V_{c b} \\
V_{t d} & V_{t s} & V_{t b}
\end{array}\right]
$$

The matrix is constructed to be unitary so naturally the sum of the modulus squared of any row or column should be equal to 1 . That is to say,

$$
V_{i j}^{*} V_{k j}=\delta_{i k}
$$




$$
V_{j i}^{*} V_{i k}=\delta_{j k} .
$$

For $\mathrm{n}$ generations of quarks the matrix $\mathrm{V}$ is unitary, constructed from $n^{2}$ elements. Although there are $n^{2}$ parameters to be determined, there is the freedom to chose the phases of $2 \mathrm{n}-1$ quark fields leaving $(n-1)^{2}$ parameters. For the case of $n=3$, three Euler angles and a phase, $\delta$, remain such that

$$
V=\left[\begin{array}{ccc}
c_{12} c_{13} & s_{12} c_{13} & s_{13} e^{-i \delta_{13}} \\
-s_{12} c_{23}-c_{12} s_{23} s_{13} e^{i \delta_{13} s} & -c_{12} c_{23}-s_{12} s_{23} s_{13} e^{i \delta_{13}} & s_{23} c_{13} \\
s_{12} c_{23}-c_{12} c_{23} s_{13} e^{i \delta_{13}} & c_{12} c_{23}-c_{12} c_{23} s_{13} e^{i \delta_{13}} & c_{23} c_{13}
\end{array}\right] .
$$

The Euler Angle representation of the CKM matrix is such that $c_{i j} \equiv$ $\cos \theta_{i j}$ and $s_{i j} \equiv \sin \theta_{i j}$ and the quark families are denoted by the indices $\mathrm{i}$ and $\mathrm{j}$. The above form is the Cabibbo-Kobayashi-Maskawa representation of $\mathrm{V}[2.16]$. The values along the diagonal are close to 1 , the dominant off diagonal elements, $V_{u s}$ and $V_{c d}$, approach 0.22 , and the remaining elements are close to 0 . With $V$ in this form it is seen that $V \neq V^{*}$ implying that the phase, $\delta$, is a $\mathrm{CP}$ violating phase.

\subsubsection{Heavy Quark Effective Theory}

The predictive power of the current relativistic theory, particularly in the case of the strong interaction, is dependent on the value of the interaction coupling constants. The full relativistic theory is certainly applicable to determining the excited meson states that exclusively consist of $u, d$, or $s$ quarks. To approach to an analogous problem in QED, such as determining the hyperfine structure in positronium, one invokes a perturbative expansion in powers of $\alpha_{Q E D}$. The effectiveness of this method is rooted in the fact that $\alpha_{Q E D}$ is significantly less than one, so that higher order terms, while sometimes long and tedious to calculate, are not significant contributors to the overall solution. In relativistic QCD a perturbative expansion in powers of $\alpha_{Q C D}$ is considerably more difficult since the value of $\alpha_{Q C D}$ is much closer to one. In addition the coupling constant varies significantly more with $Q^{2}$, a 
phenomenon referred to as 'running', than $\alpha_{Q E D}$. These two properties make it attractive to find an alternative parameter on which to base a perturbative expansion.

Some of the foundations for heavy quark effective theory exist in nonrelativistic quantum electrodynamics (NRQED) in which non-relativistic momenta are treated separately from relativistic loop momenta[2.17]. In addition, bound state systems are considered exclusively non-relativistic such that only NRQED calculations are necessary. The lagrangian for NRQED contains non-relativistic two component Pauli spinor fields, $\psi$, for the electron and positron although the photon is unavoidably relativistic and is treated the same as in QED. Here is the form quoted by Caswell and Lepage(1986):

$$
\begin{aligned}
L_{e f f} & =-\frac{1}{2}\left(E^{2}-B^{2}\right)+\psi^{\dagger}\left(i \frac{\partial}{\partial t}-e \phi+\frac{\mathbf{D}}{2 m}\right) \psi \\
& +\psi^{\dagger}\left[\frac{c_{1} \mathbf{D}^{4}}{8 m^{3}}+c_{2} \frac{e}{2 m} \boldsymbol{\sigma} \cdot \boldsymbol{B}+c_{3} \frac{e}{8 m^{2}} \boldsymbol{\nabla} \cdot \boldsymbol{E}\right. \\
& \left.+c_{4} \frac{e}{8 m^{2}} i \boldsymbol{D} \cdot \boldsymbol{E} \times \boldsymbol{\sigma}\right] \psi \\
& +\psi^{\dagger}\left[d_{1} \frac{e}{8 m^{3}}\left\{\mathbf{D}^{2}, \boldsymbol{\sigma} \cdot \boldsymbol{B}\right\}\right] \psi \ldots
\end{aligned}
$$

where $\mathbf{D}=\nabla+\mathbf{i e A}$.

The coupling constants $c_{i}$ and $d_{i}$ are chosen to agree with equivalent scattering amplitudes in QED. A salient point to notice is that the effective lagrangian is expressed as an expansion in terms of $1 / \mathrm{m}$ which leads to an expansion in $\mathrm{v} / \mathrm{c}$. The heavier the particle the faster the series converges and the fewer terms are required to get a reasonable calculation. This same philosophy is practiced in non-relativistic QCD in which hadrons containing one or more heavy quarks are treated in a lattice and energy levels are calculated in a reduced zone scheme[2.18]. The NRQCD effective lagrangian has a similar form to that of NRQED with the interpretation that $1 / \mathrm{M}$ ( $\mathrm{M}$ is the quark mass) is the order of the lattice spacing. To see if the expansion in powers of $\mathbf{v}$ is valid for heavy quarks consider a system with a b quark. 
Assuming a non-relatistic velocity of $1 / 5 \mathrm{c}$ and taking $m_{b}$ as being on the order of $5 \mathrm{GeV} / \mathrm{c}^{2}$ the de-Broglie wavelength is specified by:

$$
p=m v=\frac{\hbar c}{c} \frac{2 \pi}{\lambda} .
$$

Noting that $\hbar c=197 \mathrm{MeV} \cdot \mathrm{fm}$

$$
\lambda=1.24 \mathrm{fm} .
$$

Now consider a $d$ or u quark with the same velocity and assuming a mass of $1 / 3 m_{\text {proton }}$ for simplicity. The wavelength becomes $\lambda=18.8 \mathrm{fm}$ which is larger than the radius of some nucleii. It reemphasizes the fact that the lattice constant must be small enough to feel the strong coupling.

It is relevant here to define a heavy quark, $Q$, in the framework of the minimal standard model such that $M_{Q} \gg \Lambda_{Q C D}$. Bottom and charm (to an extent) satisfy this criteria and of course the top quark, being the heaviest quark, does as well but is too short-lived to hadronize. It is fairly obvious that the heavy quark comprises most of the mass and, therefore, momentum of a heavy hadron. From this observation two symmetries are revealed which simplify the calculation of transition amplitudes involving heavy quarks $[2.19,2.20]$.

1. The heavy quark is considered a static color source in the hadron's rest frame such that the spin degree of freedom decouples from the light quark(s) generating an $\mathrm{SU}(2)$ spin symmetry.

2. An $\mathrm{SU}(2)$ heavy quark flavor symmetry also arises for processes in which one heavy hadron decays into another heavy hadron.

From 1) one observes that the properties of $B$ and $B^{*}$ mesons are related and from 2) the properties of $B$ and $D$ mesons are related. Both instances are illustrated in Figure 2.2. To generalize, if a heavy quark undergoes a transition such that $[2.21]$

$$
M_{Q} v^{\mu}=M_{Q^{\prime}} v^{\prime \mu}+q^{\mu}
$$


then in the limit of $M \rightarrow \infty$ for fixed momentum difference, $q^{\mu}$, the velocities of the initial and final quark states are equal. A new effective field theory
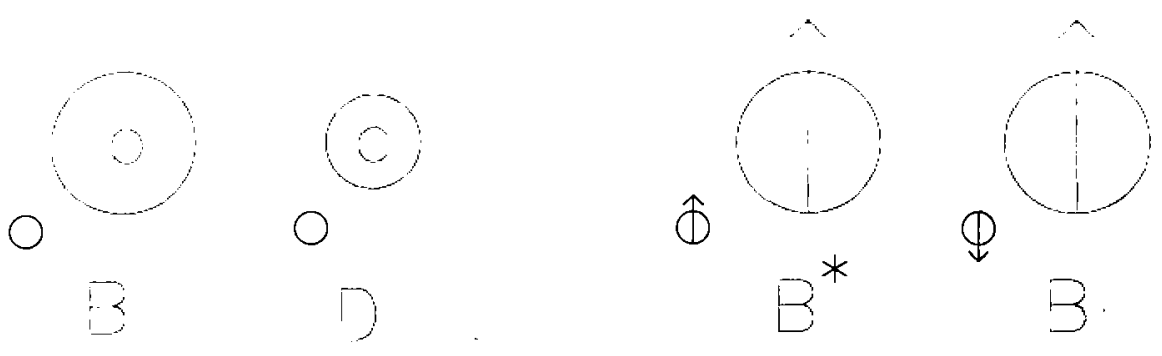

Figure 2.2: The SU(2) symmetries associated with HQET. The left side figure represents a general $\mathrm{b} \rightarrow \mathrm{c}$ transition while the right side figure shows the spectroscopy of a heavy meson.

is based on the velocity of the QCD bound state. For each velocity a new heavy quark field is created since one must transfer to the rest frame of the heavy quark to apply the $\mathrm{SU}(2)$ symmetries associated with a stationary color field. The heavy quark lagrangian in this effective theory reveals the velocity superselection rule such that a different field is constructed for each velocity[2.22]:

$$
\mathcal{L}=\sum_{h} \int_{v} \frac{d^{3} \mathbf{v}}{2 v^{0}} L_{v}^{h}
$$

where

$$
\begin{gathered}
L_{v}=i h_{v}^{\dagger} \gamma^{\circ}\left(\gamma^{\mu} v_{\mu}\right) v_{\mu} \partial^{\mu} h_{v} \\
h_{v}(x)=e^{i M\left(\gamma^{\mu} v_{\mu}\right) v_{\mu} x^{\mu}} \psi(x)
\end{gathered}
$$

with the field commutation relation,

$$
\left[h_{v}, h_{v^{\prime}}^{\dagger}\right]=\left(\gamma^{\mu} v_{\mu}\right) \gamma^{\circ} \delta_{v, v^{\prime}}
$$


Hadrons containing one or more $b$ quarks seem to be the best area to test the predictive power of HQET. With the short lifetime of top the $b$ is the heaviest quark that forms QCD bounds states with other quarks. Charm binds with other quarks to form mesons and baryons but its mass is marginally heavy. Experiments measuring the hadronic decay widths and corresponding lifetimes of charged and neutral $D$ mesons suggest that the light degrees of freedom play a greater role than that dictated by HQET. The decay width of the neutral $\mathrm{D}$ is a factor of 2.7 greater than the width of the charged $\mathrm{D}$ and the lifetime ratio $\mathrm{D}^{+} / \mathrm{D}^{\circ}$ is about $2.1[2.23]$. The charged and neutral B mesons, however, have very similar masses and lifetimes, both suggested by HQET. In essence, the $b$ sector provides a mass and energy range in which HQET is applicable while charm sets somewhat of a lower limit on its viability.

Study of the $b$ sector is also important in the phenomena of CP violation. It has been already observed for the neutral $\mathrm{K}$ system and is predicted to be an even more pronounced effect for neutral $\mathrm{B}$ mesons. In particular the $\mathrm{B}_{d}$ $\rightarrow \mathrm{J} / \psi \mathrm{K}_{\text {a }}^{\circ}$ decay leads to a possible time integrated asymmetry[2.24],

$$
\begin{gathered}
A(f)=\frac{\Gamma\left(B_{t=o}^{o} \rightarrow f\right)-\Gamma\left(\overline{B_{t=o}^{o}} \rightarrow f\right)}{\Gamma\left(B_{t=o}^{o} \rightarrow f\right)+\Gamma\left(\overline{B_{t=o}^{o}} \rightarrow f\right)} \\
A\left(J / \psi K_{s}^{o}\right)=-\frac{x_{d}}{1+x_{d}^{2}} \sin (2 \beta)
\end{gathered}
$$

where $x_{d}=\frac{\Delta m}{\Gamma}$ evaluated for the $B_{d}$. To observe this asymmetry directly requires high statistics and knowing if the $\mathrm{B}^{\circ}$ contains $a b$ or $\bar{b}$ quark. An alternative method that may be used in the future is to look at the exclusive hadronic decays of $B^{* *}$. Should the decay proceed

$$
B^{* *} \rightarrow \pi B
$$

then the charge of the pion would act to tag the $b$ from the $B^{* *}$ and its lineage could then be traced to the B meson. Many of the event selection features of 
the exclusive lifetime analysis would be used in order to determine properties of the B's from B**. Strong evidence for B $\pi$ correlations leading to resonant $\mathrm{B} \pi$ production has been observed at LEP [2.25] using inclusive $\mathrm{B}$ decays in which the b quarks emanate from their high statistics sample of $Z^{\circ} \rightarrow b \bar{b}$. 


\section{Bibliography}

[2.1] Lattes, Occhialini, and Powell, Observations on the tracks of slow mesons in photographic emulsions, Nature. 4066 (1947) 453.

[2.2] Steinberger, Panofsky, and Steller, Evidence for the production of neutral mesons by photons, Phys Rev. 786 (1949) 802.

[2.3] $\theta$ and $\tau$ were the nomenclatures at the time. The translation of $\theta$ is $K_{2 \pi}$ and $\tau$ is $K_{3 \pi}$ according to present standards.

[2.4] Brown, et al., Observations with electron-sensitive plates exposed to cosmic radiation, Nature. 163 (1949) 82.

[2.5] Gell-Mann M. Phys. Rev. 125 (1962) 1067.

[2.6] A. Pevsner, et al., Evidence for a Three-Pion Resonance near $550 \mathrm{Mev}$, Phys. Rev. Lett. 11 (1961) 138

[2.7] V. E. Barnes, et al., Observation of a Hyperon with Strangeness Minus Three, Phys. Rev. Lett. 12 (1964) 151.

[2.8] The CDF Collaboration, Evidence for Top Quark Production in $\bar{p} p$ Collisions at $\sqrt{ } s=1.8 \mathrm{Te} V$, Fermilab-Pub-94/116-E

[2.9] Left handed to conform with the 'handedness' of neutrinos observed $\beta$ decay.

[2.10] S. L. Glashow, Nucl. Phys. 22 (1961) 279. 
[2.11] A. Salam, Elementary Particle Theory, ed. N. Svartholm, Stockholm, Almquist and Wiksells. (1968).

[2.12] S. Weinberg, Phys. Rev Lett. 19 (1967) 1264.

[2.13] UA 1 Collaboration, Experimental observation of isolated large transverse energy electrons with associated missing energy at $\sqrt{ } s=540 \mathrm{GeV}$, Phys. Lett. 122B (1983) 103.

[2.14] UA 1 Collaboration, Experimental Observation of Lepton Pairs of Invariant Mass around $95 \mathrm{GeV} / \mathrm{c}^{2}$ at the CERN SPS Collider, Phys. Lett. 126B (1983) 398.

[2.15] P. W. Higgs, Phys Lett, 12 (1964) 132.; P. W. Higgs, Phys Rev, 145 (1966) 1156.

[2.16] N. Cabibbo, Phys. Rev. Lett. 10 (1963) 531.; M. Kobayashi and T. Maskawa, Prog. Theor. Phys. 49 (1973) 652.

[2.17] W. E. Caswell and G. P. Lapage, Phys. Lett. 167B (1986) 437.

[2.18] G. P. Lepage and B. A. Thacker, Effective Lagrangians for Simulating of Heavy Quark Systems, Nucl. Phys. B 4 (1988) 199.

[2.19] N. Isgur and M. Wise, Weak Decays of Heavy Mesons in the Static Quark Approximation, Phys. Lett. B 232 (1989) 113.

[2.20] N. Isgur and M. Wise, Weak Transition form Factors between Heavy Mesons, Phys. Lett. B 237 (1990) 527.

[2.21] H. Georgi, An Effective Field Theory for Heavy Quarks at Low Energies, Phys. Lett. B 240 (1990) 447.

[2.22] A. Falk, H. Georgi, B. Grinstein, and M. Wise, Heavy Meson Form Factors from $Q C D$, Nucl. Phys. B 343 (1990) 1. 
[2.23] Peter Renton, Electroweak Interactions: An introduction to the Physics of Quarks and Leptons., 1990 413-420.

[2.24] J. L. Rosner, The Cabibbo-Kobayashi-Maskawa Matrix, B Decays, ed. S. Stone (1994).

[2.25] The OPAL Collaboration, Observations of $\pi-B$ charge-flavor correlations and resonant $B \pi$ and $B K$ production, CERN-PPE/94-206. 


\section{Chapter 3}

\section{Hardware and Software}

The equipment at Fermilab and, in particular, CDF are designed to observe a wide variety of particles. The physics often pushes hardware and software technology to its limits to measure properties of the rare particulates of matter. This chapter describes the accelerator stages, CDF detector, trigger levels, and some of the supporting offline code used in track reconstruction.

\subsection{The Accelerator}

The acceleration of protons to their maximal energy occurs over five stages. The first stage involves a Cockcroft-Walton generator which is equivalent in many ways to a basic voltage multiplier. The cycling frequency is high such that its inverse is small compared with the RC time constant for the circuit in Figure 3.1. The capacitors never fully discharge and the potential accumulates until it reaches the tolerated limit. The electrostatic accelerator reveals two sets of capacitors connected in parallel by a diode array. Typically, the values of $C_{i}=C$ and the number of layers, $\mathrm{n}$, is kept to a minimum since the voltage fluctuation at the output varies as $n^{3}[3.1]$. Atomic hydrogen is combined with electrons to form $\mathrm{H}^{-}$and the ions are accelerated through 750,000 electron volts to the next stage. 
The Linac forms stage 2 of the acceleration process. The klystrons are similar to microwave generators used in radar technology. The transverse instability in the resonant cavity is a function of $\left(1-\beta^{2}\right)$ so that for particles (i.e. ions) with non-relativistic velocities the beam becomes dispersed over



Figure 3.1: Simplified schematic for a Cockcroft-Walton electrostatic generator.

substantial lengths. To mitigate this problem, ions are accelerated in discrete regions within the resonant cavity. Drift tubes carry the ions down the Linac to the next acceleration region till they ultimately reach the desired energy. A simplified diagram of an Alvarez Linac is shown in Figure 3.2. As an ion achieves higher velocities, the drift tubes become 1) longer in order to account for the increase in distance traversed for the same RF period and 2) narrower to maintain the equality between resonant frequency of the cavity and the electrostatic accelerating field. At Fermilab the Linac is about 500 feet long containing 9 drift tubes originally, although an upgrade has replaced the last four tubes. The negatively charged hydrogen ions are accelerated to 400 


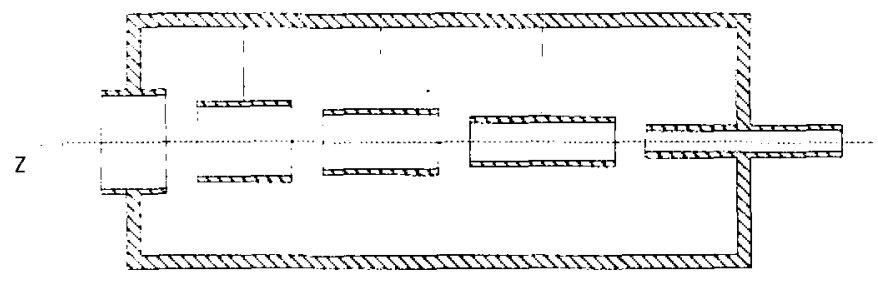

Figure 3.2: Generalized model of a cylindrical Alvarez Linac. The drift tubes are encased in the waveguide cavity and the acceleration occurs in discrete steps between the drift tube edges. 
$\mathrm{MeV}$ and the electrons are then stripped away by passing the ions through a carbon foil.

The denuded protons are ready for acceleration within stage 3 , the Booster. The Booster is a rapid cycling synchrotron 500 feet in diameter. It is comprised of east and west RF control stations having 7 and $10 \mathrm{RF}$ cavities, respectively. As protons pass through the two gaps contained within each

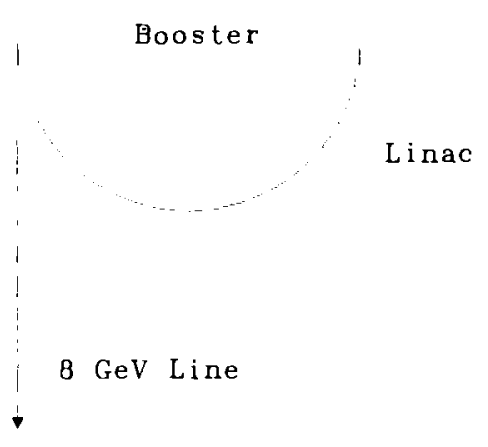

Figure 3.3: Schematic of the Fermilab Booster.

cavity an alternating RF electric field is applied that increments from 38 $\mathrm{MHz}$ up to $52.81 \mathrm{MHz}$. After 20,000 orbits within the Booster the protons reach an energy of about $8 \mathrm{GeV}$. The momentum compaction, $\frac{d P / P}{d S / S}$ where $S=2 \pi R_{\text {booster }}$, is relatively small for the Booster such that each of the 12 booster cycles produces one group of protons or bunch. The transverse beam dimensions are tracked through each turn in the Booster by an Ion Profile Monitor. Figure 3.3 shows the simplified schematic of the Booster in relation to the position of the Linac and Figure 3.4 is a snapshot of an RF cavity.

The proton bunches then enter the Main Ring which comprises stage 4 


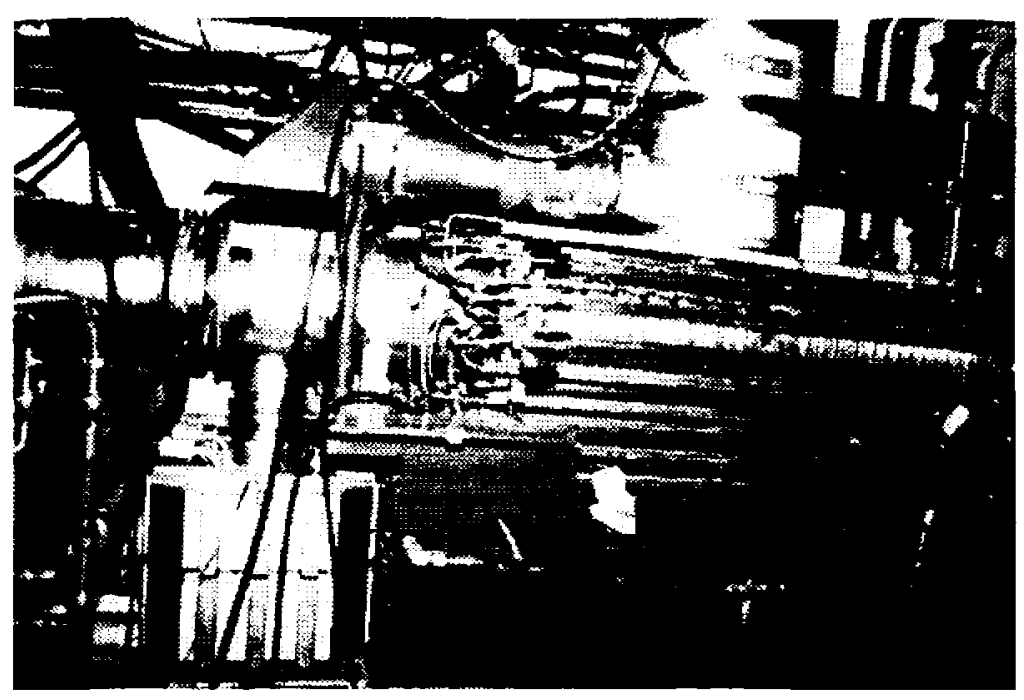

Figure 3.4: A sample snapshot of one of the RF cavities within the Booster.

of the acceleration process. The Main Ring is simply a substantially larger version of the Booster. The synchrotron is approximately 4 miles in circumference and contains 1000 regular, or nonsuperconducting, magnets that alternately bend and focus the proton bunches. Many of the same RF control systems that monitor Booster operation also monitor the Main Ring's 18 RF stations. A Fast Bunch Integrator is used to measure the proton bunch intensity. Transfer of the proton bunches from the Main Ring to stage 5, the Tevatron, occurs via a Cogging Low Level RF system. The phases and frequencies between the Main Ring and Tevatron RF cavities are synchronized by a method similar to that of a Phase Locked Loop (PLL) but more sophisticated to minimize transients in the feed back loops that are inherent to PLL's[3.2]. The Tevatron takes the $150 \mathrm{GeV}$ protons from the Main Ring and accelerates them to nearly $1 \mathrm{TeV}$. In structure the Tevatron is very similar to the Main Ring as the 1000 liquid He cooled superconducting magnets that make up the Tevatron are set in the same tunnel as the Main Ring. 


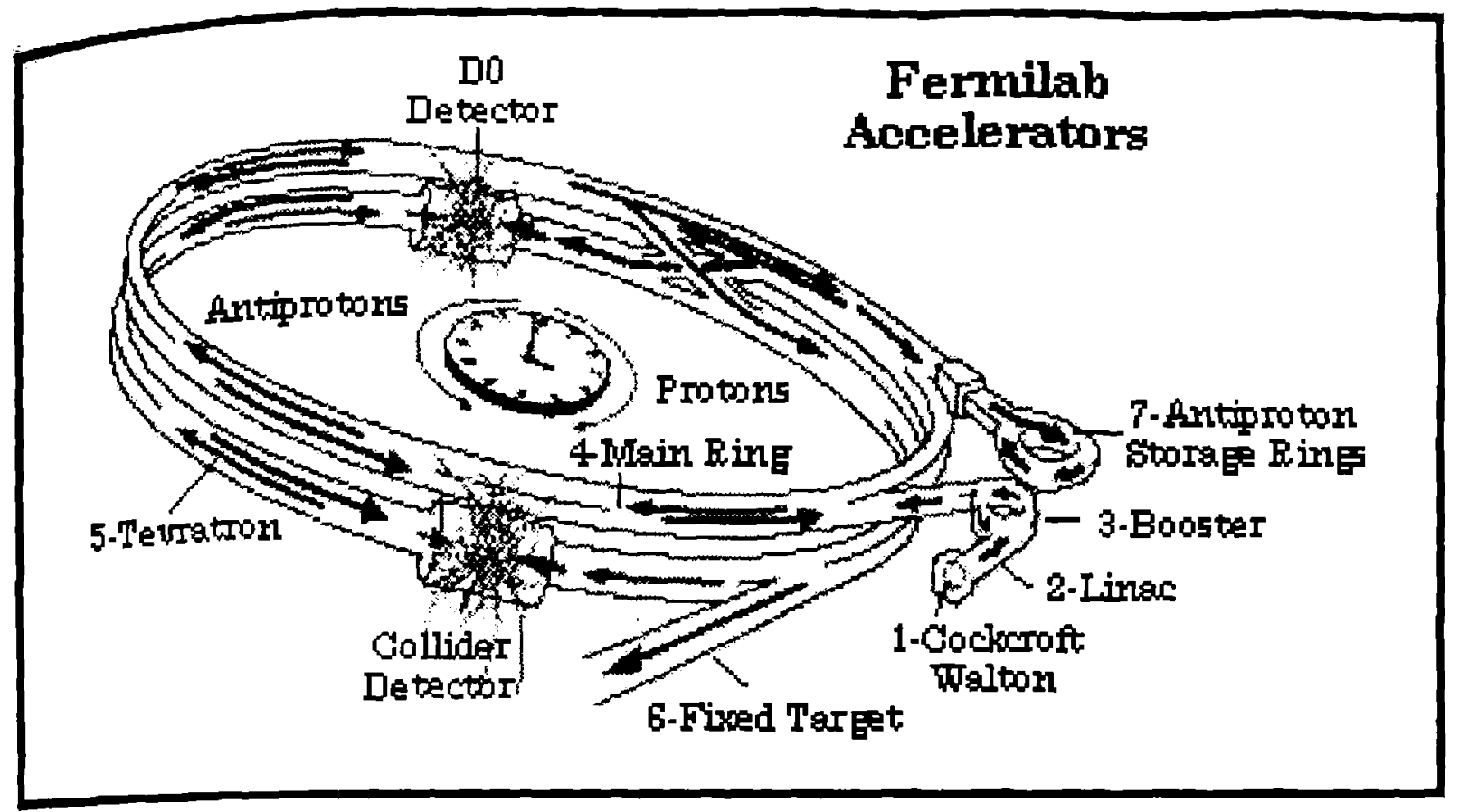

Figure 3.5: Schematic of the 5 acceleration stages including the CockcroftWalton generator, Linac, Booster, Main Ring, and Tevatron. The proton and antiproton bunches are brought into collision at the D0 and CDF (B0) detectors. 
Antiprotons are generated by colliding $120 \mathrm{GeV}$ protons from the Main Ring into a fixed copper target. After they undergo stochastic cooling the antiprotons are stored in an accumulator ring until a sufficient number accrue. The process of creating and storing antiprotons is known as stacking. The stack of antiprotons is then injected back into the Main Ring eventually finding its way into the Tevatron where they are accelerated to the same energy as the protons but traveling in the opposite direction within the Tev ring. Figure 3.5 shows the relative location of the 5 acceleration stages from one another including the antiproton storage ring.



Figure 3.6: An isometric expanded view of the CDF detector. 


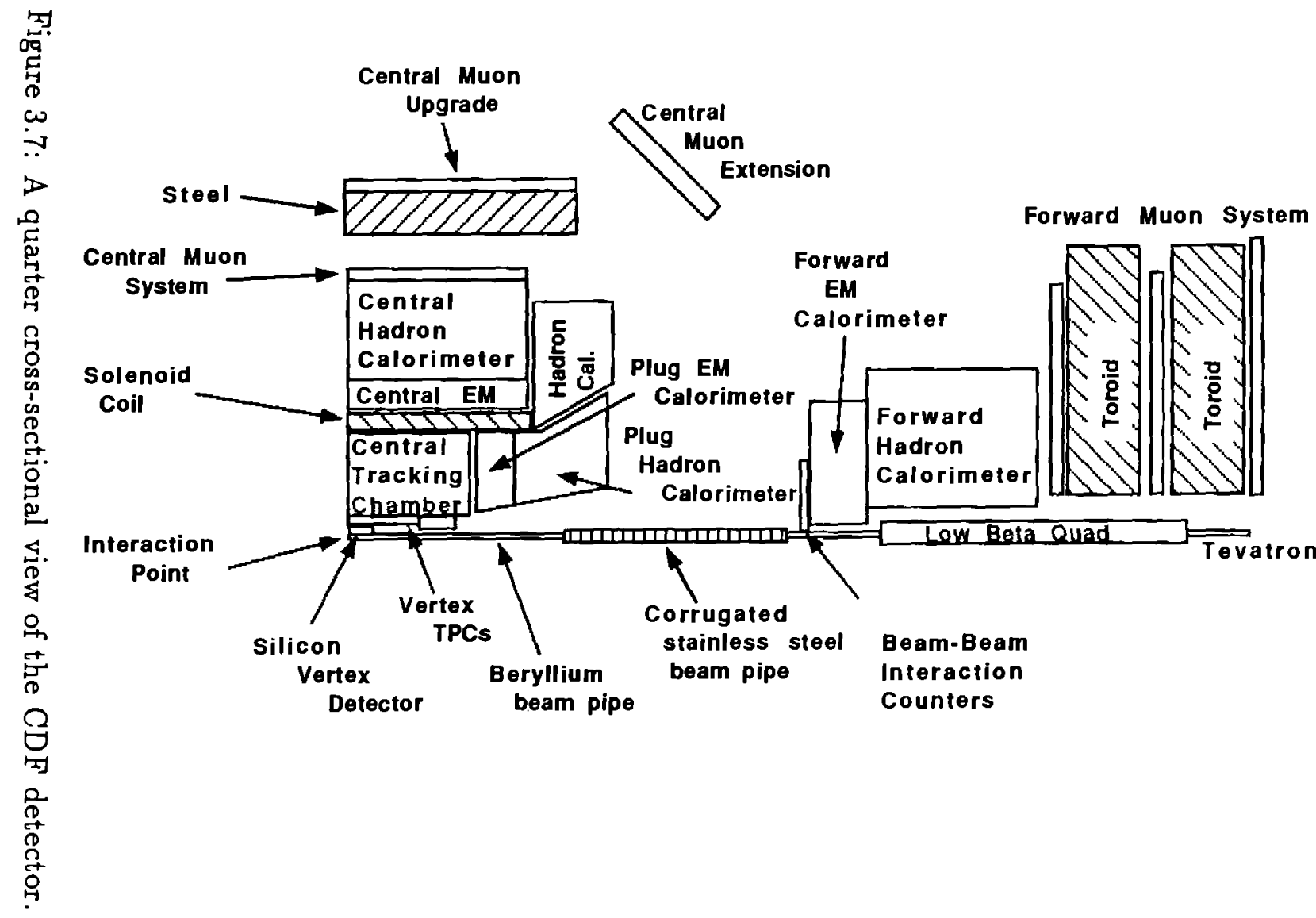




\subsection{The Detector}

As seen in Figure 3.5 the $3.5 \mu \mathrm{m}$ spaced proton and antiproton bunches collide at 2 interaction sites, D0 and B0. The detector at B0, commonly referred to as $\mathbf{C D F}$, has been described in various articles according to the upgrades that have taken place since 1988[3.3]. The detector consists of many features but, for expediency, only those relevant to the $\mathrm{B}$ meson candidate selection need discussion in some detail.

\subsubsection{Muon Chambers}

Figures 3.6 and 3.7 reveal the CDF detector components and relative positions to one another in the assembly. The muon chambers are located behind the rest of the detector components and are divided into three sections according to their geometrical coverage: CMU, CMP, and CMX. The CMU (Central MUon) chambers are cylindrically arranged around the beam axis spanning $2 \pi$ in $\phi$ modulo any gaps. Each CMU wedge spans $12.6^{\circ}$ in $\phi$ and is located behind the hadronic calorimetry approximately 3.47 meters from the beam line. As seen in the front view of Figure 3.8 the CMU wedges consist of 3 distinct units each covering a third of the overall $12.6^{\circ}$. The units themselves are a $4 \times 4$ array of rectangular drift cells with a $50 \mu \mathrm{m}$ sense wire located at the center of each drift cell. The CMP (Central Muon uPgrade) box-like formation behind the CMU increases coverage in $\phi$ by overlapping with the $2.4^{\circ}$ gaps between adjacent CMU wedges. It also serves to validate muons seen by the CMU since the coverage of the CMU and CMP are almost identical. Plus, the CMP sits behind almost 9 interaction lengths of material as opposed to the CMU's 5. Signals emanating outside (i.e. cosmics) seen by the CMU are reduced by the extra iron material between the CMU and CMP. The four CMX (Central Muon eXtension) arches provide additional longitudinal muon coverage in the range from $55^{\circ}>\theta>36^{\circ}$. Essentially,

none of the coverage overlaps with either the CMU or CMP. 
A detailed description of muon chamber performance is described in reference 3.4 but some of the salient features are outlined here for completeness. The drift cells are constructed of aluminum in which the radial sides are
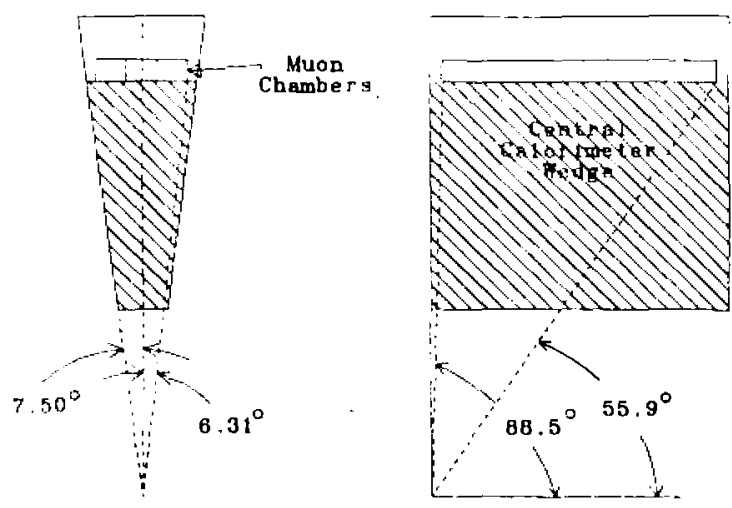

$\mathbf{Y} \uparrow$
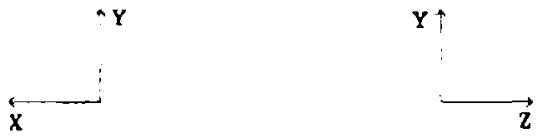

Figure 3.8: Front and side views of a typical CMU wedge behind the hadronic calorimetry.

$25.4 \mathrm{~mm}$ long. The angular ends are $.79 \mathrm{~mm}$ thick and layers are insulated from one another by a layer of $.62 \mathrm{~mm} \mathrm{G}-10$ strips. A potential of $-2500 \mathrm{~V}$ is maintained at each radial side. Since the sense wire is only $50 \mu \mathrm{m}$ thick and spans the entire $2260 \mathrm{~mm}$ length of each cell, the wire must by restricted from bowing by $110 \mathrm{~g}$ of tension. The ionizing gas in the muon chambers is a $50 \% / 50 \%$ argon-ethane mixture existing in an electric field of at least $100 \mathrm{~V} / \mathrm{mm}$ ensuring a constant drift velocity throughout the cell. Figure 3.9 shows the cross sectional view of a typical muon drift cell chamber. 


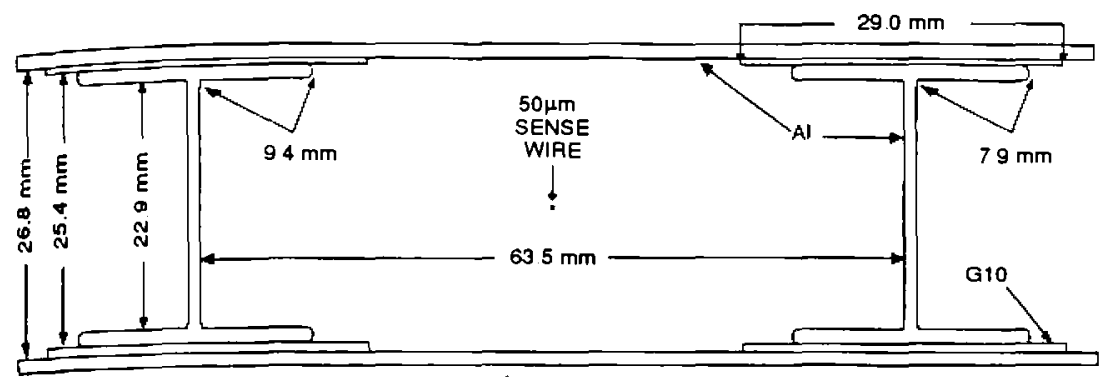

Figure 3.9: A typical drift cell.

The position of a track is determined by the relative charge deposited along the length of the cells' sense wires. Figure 3.10 shows the connection between alternating drift cell layers in which the connection allows for Redundant Analog Bus-Based Information Transfer, or RABBIT, muon ADC-TDC electronics to be placed at the $\theta=56^{\circ}$ end of the layer assemblies. For charge, $Q_{i}$, collected on wire $1 \mathrm{X} \mathrm{mm}$ away from the $\theta=90^{\circ}$ end the charge reaching $\mathrm{ADC1}$ is

$$
Q_{1}=Q_{i} / 2\left(1+\frac{X}{L} e^{-t / \tau}\right)
$$

where $\mathrm{L}$ is the wire length, and for wire 2 the charge gathered by $\mathrm{ADC} 2$ is

$$
Q_{2}=Q_{i} / 2\left(1-\frac{X}{L} e^{-t / \tau}\right)
$$

where $\tau$ is the RC time constant of the wire owing to its finite $50 \Omega$ resistance and the $0.1 \mu \mathrm{F}$ input blocking capacitor to the ADC's. The relative charge 
deposition is the ratio of the two,

$$
R=Q_{2} / Q_{1}=\frac{X}{L} e^{-t / \tau}
$$

The ${ }^{55} \mathrm{Fe}$ sources in the existing radial sides are used to calibrate this charge division.

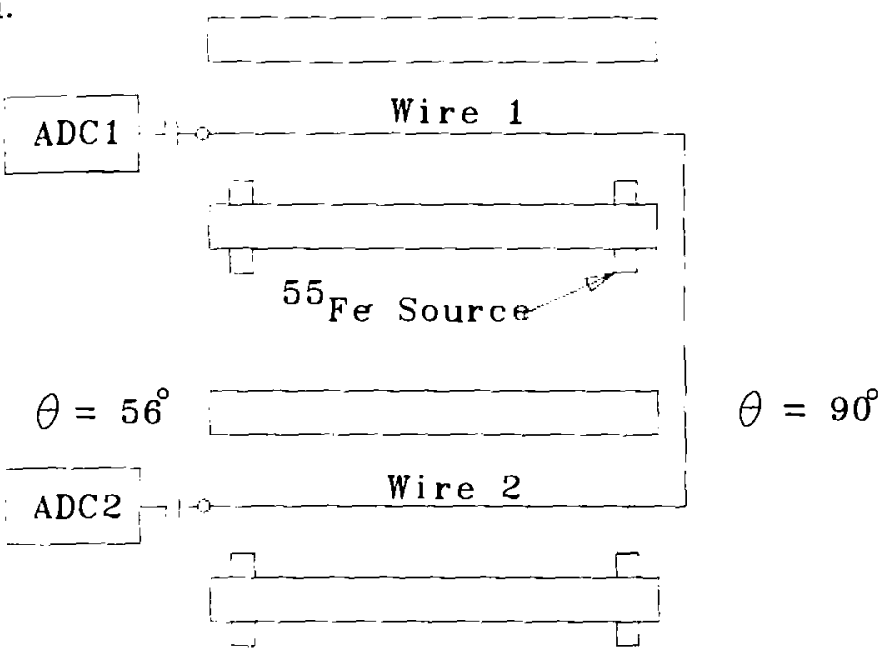

Figure 3.10: Side view of three layers in which the connection between wires 1 and 2 allows for the placement of their respective ADC's on the $\theta=56^{\circ}$ end of the calorimeter wedge.

\subsubsection{Central Tracking Chamber}

Inside the solenoid lies the Central Tracking Chamber or CTC. The CTC's $2 \pi$ azimuthal acceptance is easily seen in Figure 3.6. It is constructed of 84 layers of wire chambers arranged as interleaved 5 axial and 4 stereo superlayers. The stereo cells are offset $\pm 3^{\circ}$ with respect to the beam axis. Each 
axial superlayer is equivalent to 12 sense wire layers while the stereo superlayers consist of 6 layers. The wires are tilted $45^{\circ}$ to compensate for the large Lorentz angle dictated by the magnetic field combined with the nearly 1350 $\mathrm{V} / \mathrm{cm}$ drift field. This large tilt angle is advantageous as adjacent wire layers overlap at the ends as seen in Figure 3.11.



Figure 3.11: Axial view of the Central Tracking Chamber.

As in the case with the muon chamber gas, the ionizing gas circulating through the CTC is highly volatile, being composed of a $50 \% / 50 \%$ argonethane combination that is bubbled through $-7^{\circ}$ ethanol introducing about an $.8 \%$ dopant of alcohol into the mixture[3.5]. The endplate is constructed of 2 inch thick aluminum. Wire assemblies vary in both size and material according to the function that each performs. The sense wires consist of 40 $\mu \mathrm{m}$ thick gold plated tungsten while the potential, field, guard, and shaper 
wires are made of stainless steel due its work function of approximately 4.5 $\mathrm{eV}$. Diameters of the steel wires range from $140 \mu \mathrm{m}$ to $305 \mu \mathrm{m}$ and the respective tension under which each is held increases as a function of diameter from $661 \mathrm{~g}$ to $1259 \mathrm{~g}$. The relative locations of all wire classes are shown in Figure 3.12. One interesting feature is that the individual MWPC layers use

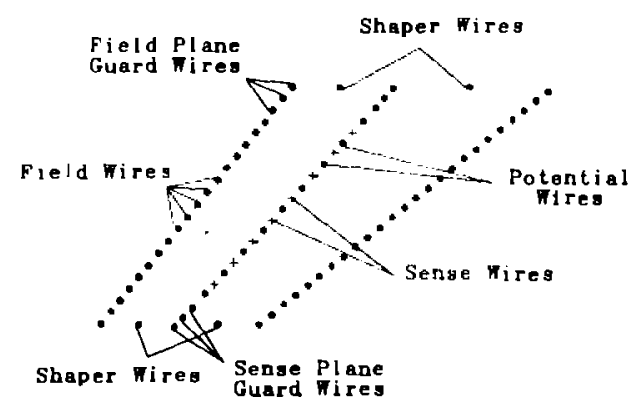

Figure 3.12: Location of the major wire classes relative to the sense wires in the axial layers of the CTC. The shaping wires help to manage the nonlinearity of the drift field at the edges of each layer.

field wires instead of a single plate in order to obtain better control over the linearity of the electrostatic drift field. The potential wires spaced between the sense wires allow for changes in gas gain.

Charge reaching the wires is first read in as signal to a preamplifier card located on the CTC enplate. A network of resistors is used to reduce crosstalk among adjacent sense wires prior to any charge to current conversion in the preamp circuitry. Knowledge of the amount of charge that bleeds to the nearest neighbor wires determines the resistor values. Rated at a breakdown of $3 \mathrm{kV}, 1200 \mathrm{pF}$ blocking capacitors provide the connection between the sense wires and the crosstalk network leading up to the preamplifier. 
From the preamp the signal is then shaped and further amplified before it is received by a Fastbus TDC (Time to Digital Converter) controlled by the newer FRC's (Fastbus Readout Controller) replacing the SLAC Scanner Processors (SSP's). The high fidelity of this device was demonstrated by its survival of Run's $1 \mathrm{~A}, 1 \mathrm{~B}$, and all but two weeks of Run 1C. Deterioration of the inner layers was inevitable, but this effect was negligible due in part to the excellent resolution of the SVX'.

\subsubsection{The Silicon Vertex Detectors}

This component of CDF hardware is the most important when discussing any analysis involving vertexing because of its proximity to the beam pipe. During the course of Run I two silicon vertex detectors were installed, the SVX in 1991 and its replacement SVX' in 1993. Comparing the two detectors yields some obvious similarities[3.6]. Both are comprised of east and west barrels such that their point of union resides at the nominal $\mathrm{z}=0$ position of the collisions. Each were constructed from 4 layers arranged in a twelve sided geometry such that each active area within a wedge subtends approximately $30^{\circ}$ in $\phi$. Figure 3.13 shows an isometric view of the SVX' detector. Twelve ladders make up one layer and each ladder has three silicon detectors connected on the short sides, each glued to Rohacell[3.7] with a pair of carbon fiber tracks underneath as seen in Figure 3.14. Beryllium bulkhead materials were chosen for their low mass although the hazards inherent to the machining process of beryllium is somewhat expensive. The $z$ coverage for both SVX and SVX' remains at $51 \mathrm{~cm}$. Just as important for the continuity of transition between data taken with the SVX and SVX' is that several institutions contributed to the design of both detectors.

Several assembly and electronics upgrades included with the SVX' contribute to its superior performance over the SVX. The innermost layer (L0) for the SVX' is set $.1437 \mathrm{~mm}$ closer to the beam at $2.8612 \mathrm{~cm}$. SVX' L0 ladders overlap by approximately 1.5 strips which is equivalent to $.17^{\circ}$ in $\phi$ 


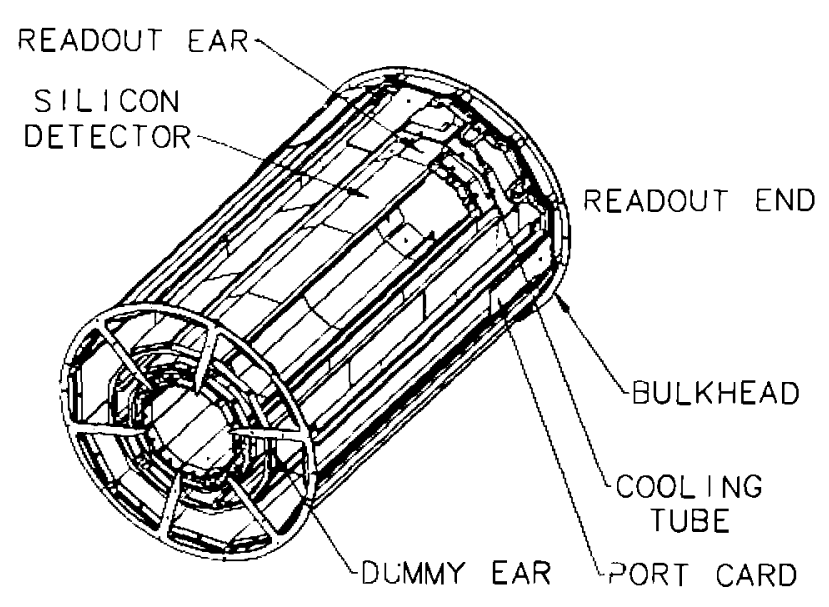

Figure 3.13: An isometrical view of an SVX' barrel.

in contrast to the $1.26^{\circ}$ gaps that exist for the SVX. During Run 1A the SVX and VTX shared a common 50\%/50\% argon-ethane atmosphere, but for Run $1 B$ the SVX' barrels contained only dry nitrogen. Single sided detectors of the SVX' have masking layers to include a Field OXide Field Effect Transistor (FOXFET) with the gate fabricated at the readout end and a capacitive dielectric layer between the $\mathrm{p}^{+}$implants and the aluminum microstrips seen in Figure 3.15. The active part of the detector is an array of separate $\mathrm{p}-\mathrm{i}-\mathrm{n}$ diodes operated in reverse bias mode. Layer 0 detectors contain 256 such diodes each pitched $60 \mu$. Detectors in the outer layers correspondingly have more channels, 384 for layer 1 and 512 for layer 2 each pitched $60 \mu \mathrm{m}$, and 768 for layer 3 at a $55 \mu \mathrm{m}$ pitch.

The FOXFET provides a dynamic resistance giving the freedom to vary the potential of the $\mathrm{p}^{+}$side of the detector. Typical settings for Run $1 \mathrm{~B}$ were to tie the gate and drain to ground. The capacitive structure means 


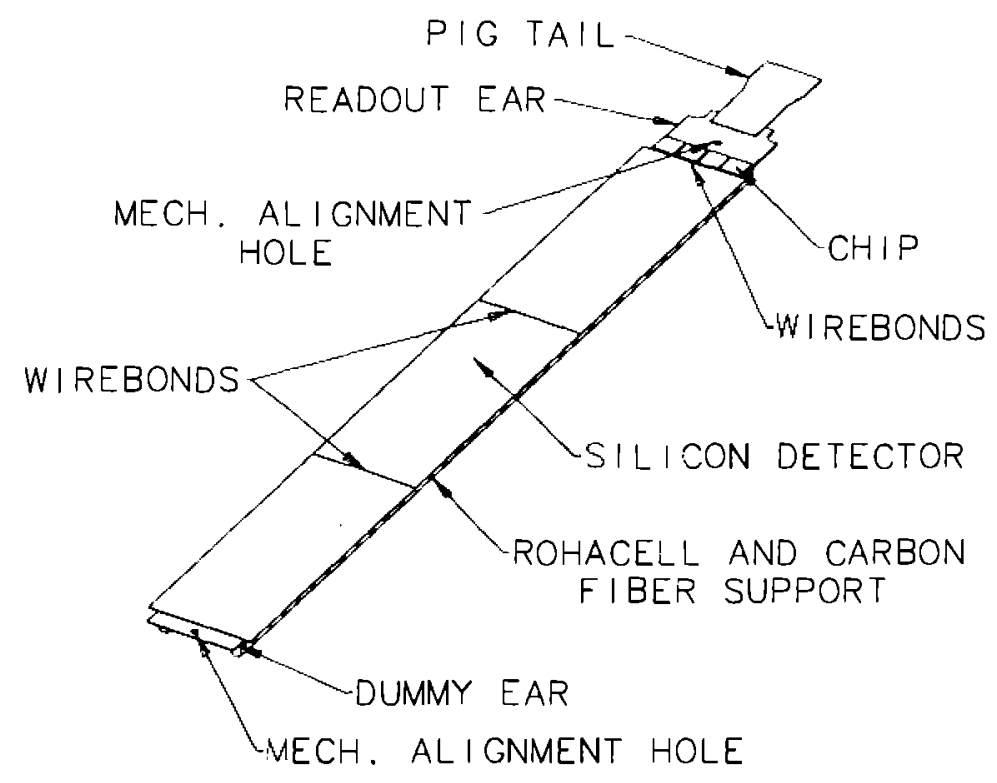

Figure 3.14: Portrait of an SVX' ladder.

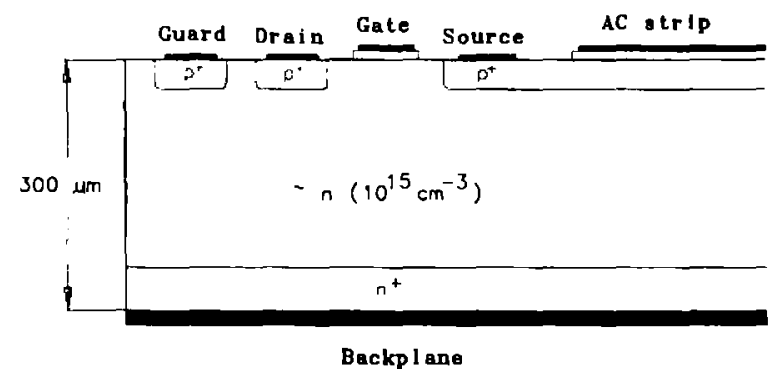

Figure 3.15: Readout end schematic cross section of an AC coupled detector. 
SVX' detectors are AC coupled verses the DC coupling of the SVX detectors in which the metalization layer is set directly over the $\mathrm{p}^{+}$implants with no intervening dielectric. Signals are received by the SVXH3 chip operated such that the charge is integrated only once taking full advantage of the AC coupling. Comparing with the DC coupled detectors bonded to older SVXD chips, two integrations are performed. One integration obtains a baseline leakage current level, and the second includes signal riding on the leakage current. The two levels are subtracted to arrive at the pulse due exclusively to signal. Although radiation exposure tends to increase dark currents, the $\mathrm{AC}$ coupling renders detector performance fairly immune to large doses since it is the relative change in signal that is passed to the preamp. Shot noise increases with prolonged exposure, but with the radiation hard CMOS SVXH3 chip combined with the SVX' detectors, the system's radiation limit is at least a factor of 50 greater than that of the SVX.

Cooling tubes containing chilled water attached to the bulkheads act as heat sinks dissipating the power generated by the electronics (of which the SVX chips are the primary contributor) bonded to the detectors. For Run 1B water is chilled to about $10^{\circ} \mathrm{C}$ and circulated through the pipes to bring the ambient temperature of the bulkhead in the range of $21^{\circ}$ to $24^{\circ}$. Care had to be taken in order to power up or down the SVX' so that the detector was not powered up without cooling or vice versa. During power cycling, a balancing act is performed so that the temperature change is smooth over time so as to allow the components to expand and contract gracefully.

\subsubsection{Trigger Levels}

All the detector components are designed to receive signals corresponding to the $3.5 \mu \mathrm{m}$ beam crossings with minimal dead time between decisions to accept or fail[3.8,3.9]. A three level trigger system filters events of interests so that these are eventually written to tape. The first two levels of the trigger system are predominantly hardware triggers while the third level is mainly 
software driven. The level one trigger selection is strongly based on energy deposition in the calorimeters. Although the $\mathrm{B}$ analysis does not depend on this type of level 1 trigger it is mentioned for completeness. At the level 1 stage the specifics of which wedge obtain hits are not sifted yet, but rather for expediency the energy deposition is analog summed using ten FASTBUS crates. Kinematics of transverse missing energy are also considered as $E_{t}$, $E_{t} \sin \phi$, and $E_{t} \cos \phi$ are summed globally for the electromagnetic and hadronic calorimetry.

Level 1 decisions are also made by the presence of stiff CTC hits. A fast track finder can also determine whether these hits are in successive CTC layers indicative of real tracks. For the lifetime analysis the most important of the level 1 triggers are the muon triggers. These are designed to trigger on known benchmark decays for example, $Z \rightarrow \mu \mu, W \rightarrow \mu \nu$, and particularly $\psi \rightarrow \mu \mu$. Other level 1 triggers include Beam-Beam counters that consist of 16 time-of-flight scintillation counters arranged in two sets. The innermost layer is located on the beam pipe. The level 1 triggers take an unfiltered event rate of $50-75 \mathrm{kHz}$ and reduce it to a couple of $\mathrm{kHz}$.

Once a level 1 accept is established the event is passed to the level 2 trigger. While the level 1 trigger incurs virtually no dead time, the level two decision making process is longer due to increased scrutiny of the various hits within the detector. Within the calorimetry level 2 investigates clustering of hits in specific towers. Various kinematic quantities are calculated such as the transverse energy, mean pseudorapidity, and mean azimuth of the cluster. Relevant to the lifetime analysis is the level 2 decision involving the Central Fast Tracker (CFT). Stiff CTC tracks are searched to see if they point to muon chamber hits in a muon wedge. The muon is labelled as 'golden' provided the previous condition is satisfied, the transverse momentum of the track is stored, and the label of CMU or CMX is associated with the track. The majority of the level 2 triggers based on the CFT were used to select dimuon events. The level 2 CFT triggers are fundamentally different in their decision making for dimuon candidates in Run $1 \mathrm{~A}$ and Run $1 \mathrm{~B}$. For $1 \mathrm{~A}$ one 
CFT track with $p_{T}>3.0 \mathrm{GeV} / \mathrm{c}$ is required to point to a muon hit cluster (a level 1 muon stub) and the second track prerequisite is only to have a muon hit cluster. The CDF jargon used to describe this type of trigger is titled

\begin{tabular}{|c|l|}
\hline CDF Trigger Name & Description \\
\hline TWO_CMU_TWO_CFT_2_2 & $\begin{array}{l}\text { Each of the two CMU clusters is } \\
\text { required to have CFT tracks above } \\
p_{T}>2 G e V / c \text { point to them. } \\
\text { The CMU clusters must be at least } \\
\text { one muon wedge apart. } \\
\text { Basically the same as above one } \\
\text { TWuon cluster in the CMU and in } \\
\text { the CMX with no wedge separation } \\
\text { requirement. } \\
\text { Similar again to above with clusters } \\
\text { in two separate CMX muon wedges. } \\
\text { The basic trigger used in Run 1A. A.CF_.TWO_CFT_2_2 } \\
\text { Clusters in any two part combination } \\
\text { of CMU and CMX with only one CFT } \\
\text { track above 3 GeV/c in } p_{T} \\
\text { required. } \\
\text { A trigger similar in principle to } \\
\text { the 3-1 trigger accept for a minimum } \\
p_{T} \text { of } 2 \text { GeV/c on the biased } \\
\text { leg of the dimuon pair. This is used } \\
\text { to study the 2-2 trigger efficiency. }\end{array}$ \\
\hline
\end{tabular}

Table 3.1: Level 2 trigger associated with dimuon event selection.

TWO_CMU_CMX_ONE_CFT_3_4. The dimuon events are filtered through a symmetric 2-2 trigger rather than the 3-1 trigger for $1 \mathrm{~A}$. Both tracks were required to have a $p_{T}>2 \mathrm{GeV} / \mathrm{c}$ with CFT tracks pointing to level 1 muon 
stubs in the CMU or CMX. CMX_CMU_TWO_CFT_2_2 exemplifies the naming of this type of trigger within CDF. Naturally, these triggers have some efficiency for turn on, but this discussion will be saved for later when describing the systematic uncertainties of the lifetime measurements. Table 3.1 outlines the major features of the various CFT associated level 2 triggers that filter dimuon events.

The level 3 trigger system is a predominantly software based system that prepares events for analysis 'off-line'. For the lifetime analysis, the level 3 trigger requires a level 1 and level 2 accept with an invariant mass window cut on the dimuon candidates. The mass window is typically 2.8 to $3.4 \mathrm{GeV} / \mathrm{c}^{2}$ which is seen in event monitoring systems such as TRIGMON. Further event building is passed to level 3 as well, providing specifics of other tracks different from those satisfying the triggers but coinciding with the triggered event. Events passing the level 3 triggering system are written to tape in raw form to be processed by physicists in charge of various data sets.

\subsubsection{Data Sets and Software Analysis}

Two main divisions of raw Run I data exist; Run 1A and Run 1B corresponding to data taken with the SVX and SVX' detectors. Dimuon data sets are created from this raw data in two primary output streams. Stream A data contains lower transverse momentum muons that reconstruct to a raw invariant mass in the vicinity of the $J / \psi$ mass. Stream B is created for high $p_{T}$ muons that reconstruct to invariant mass windows around the $\Upsilon$ and $\mathrm{Z}$ masses as well as including single muon events that are indicative of semileptonic $B$ decays.

The raw data is 'processed' using a combination of user specific routines interfaced with a large body of offline code. This offline code consists of a massive list of FORTRAN subroutines that have accumulated throughout the history of CDF. These routines undergo an approval process by the members of the collaboration such that common features of the many analyses 
which make use of these routines are standardized ensuring quality results. Throughout the course of Run I, several versions of offline code have been formally signed off by the collaboration to include versions 7.09, 7.11, and 7.12. As authors of code optimize or add to offline routines, new versions are a natural consequence. Plus, as new equipment is added to the CDF detector such as the replacement of the SVX with SVX' the offline routines are made compatible with these changes. For example the SVX and SVX' had different geometries. These geometries are stored in a database in which the offline code must reflect that difference so that the correct offsets are included for the appropriate data set. The version of offline used for this analysis is 7.12 . Run 1A data was initially processed using 7.09 while most of Run 1B data was processed with 7.11. Both data sets were then reprocessed with 7.12 tracking versions of the offline code.

A myriad of statistics associated with each event exist such as the curvature, impact parameter, $z$ position, positions in $\theta$ and $\phi$ for each track which are the five track parameters established at CDF. Other kinematic quantities calculable from the track parameters are also included. Taking into consideration that there are typically $20+$ tracks per event, to handle and store all these parameters between subroutines using arrays is time consuming and inefficient. A BANK structure has been created to handle large parameter lists. The bank format and access is similar to pointer lists handled in $\mathrm{C} / \mathrm{C}++$. Banks can be global or local depending on whether certain quantities stored within them are necessary for a wide sample of analyses.

The lifetime analysis uses information gathered from muon banks, labelled CMUO, and tracking banks (TRKS). From these widely used banks, local banks are created within the lifetime code. For instance, properties obtained from the CMUO banks of individual muons are combined in pairs to form local dimuon banks (DIMU). These DIMU banks contain the properties of the dimuon pair such as their raw invariant mass and components of 4-momentum. Information from the DIMU banks are passed to $\mathrm{J} / \psi$ search routines. Those events passing the $\mathrm{J} / \psi$ selection criterion have their charac- 
teristics stored in JPSI banks. Tracks from TRKS banks that pass quality cuts are stored as 'Good TRacKs' in local banks under the name of GTRK. GTRK banks are combined with JPSI banks to form BEXC banks which means B EXClusive banks. These BEXC banks contain all the kinematic and spatial properties of the $B$ mesons presented in this analysis. Figure 3.16 illustrates the logical flow of data passed from one bank to the next. All pri-

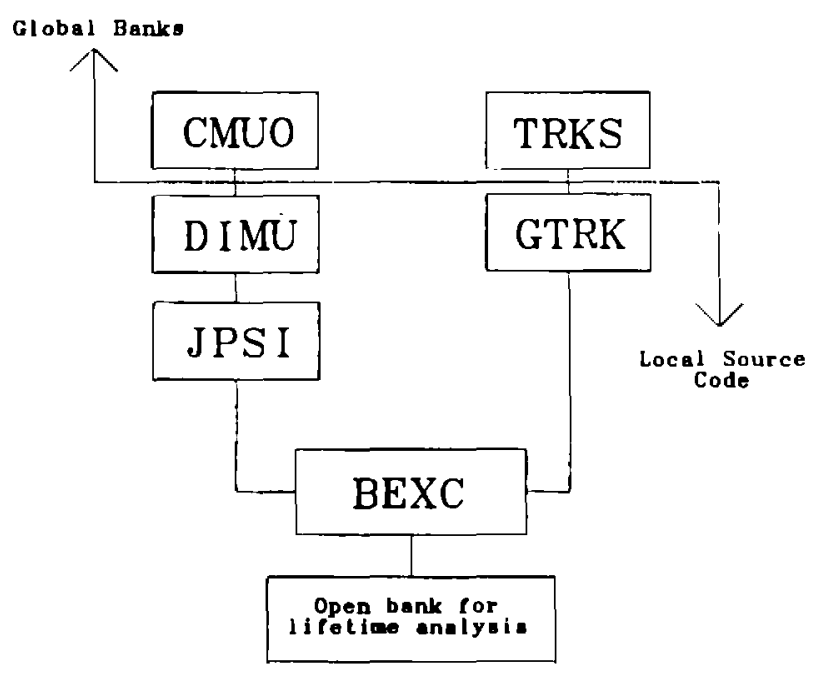

Figure 3.16: Bank flowchart leading up to the lifetime analysis.

mary routine inputs are specified within the source code or through prompts issued by Analysis/Control[3.10]. Analysis/Control provides an interactive way to run compiled code within the framework of CDF. By specifying subroutine options within $\mathrm{A} / \mathrm{C}$ it gives the freedom to alter program conditions without recompiling code. Once initial conditions are specified, the ordering of the subroutines is established by a path. The lifetime path specification is given in Figure 3.17. A somewhat pedantic explanation is given here to document the path logic as well as some of the routine names for those 


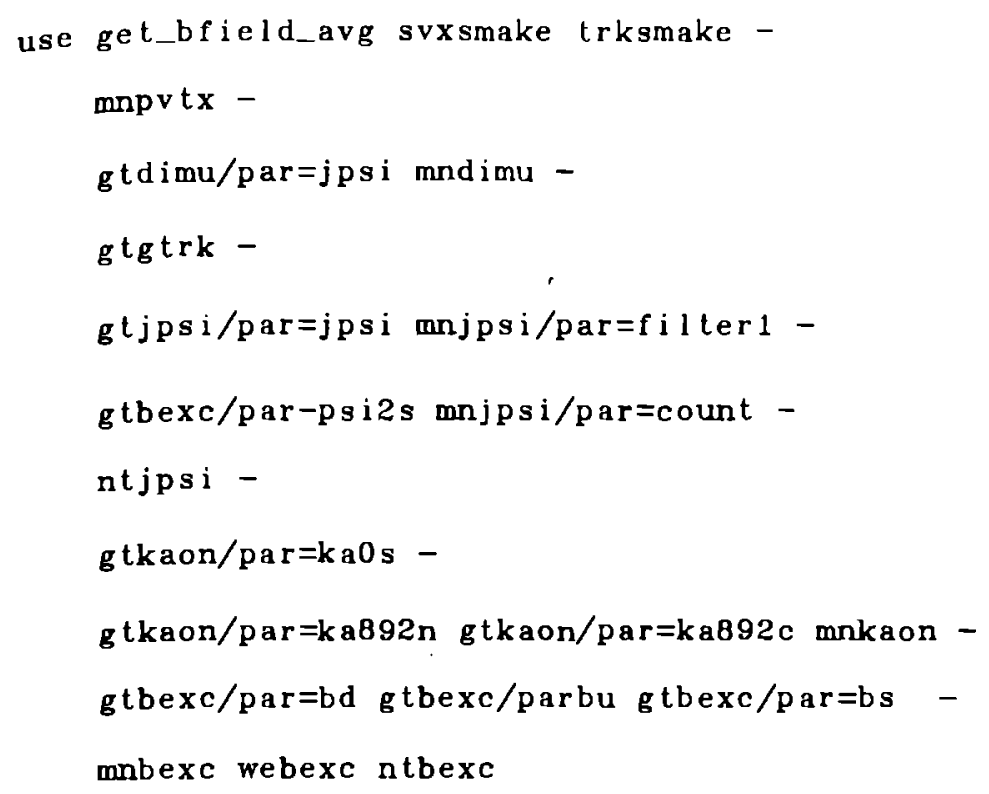

Figure 3.17: The path routines for the lifetime analysis.

who may inherit this lifetime code. The first line in the path states that a bfield correction routine is used prior to the remaking the SVXS and TRKS banks performed by SVXSMAKE and TRKSMAKE. The routine MNPVTX is called to find and monitor the primary vertex. GTDIMU is a routine that looks for dimuon pairs and creates a local DIMU bank. Initial parameters are set to look for the dimuons with raw invariant mass near the $\mathrm{J} / \psi$. There is the freedom to specify a mass in the vicinity of the $\psi(2 S)$ or $\Upsilon(1 \mathrm{~S})$, by changing $p a r=j p s i$ to $p a r=p s i 2 s$ or $p a r=u p s i l o n$. MNDIMU monitors the number of dimuon candidates by looking at how many DIMU banks were successfully created. The next couple of routines GTJPSI and MNJPSI find $\mathrm{J} / \psi$ 's and monitor the JPSI bank creation. A good track is determined by the routine GTGTRK and a bank is created for each of these with events containing a dimuon pair.

The path then specifies that the routine GTBEXC search for $\psi(2 \mathrm{~S})$ can- 
didates due to the specification, par $=p s i 2 s$. This routine searches through the GTRK banks for a pair of good tracks to combine with a JPSI bank in the same event. One can see that the routine that searches for these candidates is the same as the one that will be used to look for B candidates. The flexibility of the routine allows for different initial conditions to be set like the GTDIMU routine. The second call to MNJPSI in the path monitors the type of JPSI bank created by differentiating the $\psi$ from the $\psi(2 \mathrm{~S})$ candidates. To complete the path for $\Psi$ reconstruction, NTJPSI prepares ntuple files for analysis within PAW[3.11].

The path continues by specifying three parameters for GTKAON, ka0s, ka892n, and ka892c which stand for the $K_{a}^{\circ}, \mathrm{K}^{*}(892)^{\circ}$, and $\mathrm{K}^{*}(892)^{+}$, respectively. GTKAON creates a local KAON bank for each successful candidate by searching the GTRK banks for good tracks from which to reconstruct kaons. MNKAON monitors these banks and tags the type of kaon that exists for each KAON bank.

The final steps in the path concern $B$ reconstruction. GTBEXC specified by parameters bd, bu, or bs search for $\mathrm{B}_{d}, \mathrm{~B}_{u}$, or $\mathrm{B}_{\boldsymbol{s}}$ mesons by doing either of the following:

- Combine KAON banks with JPSI banks for the same event and create BEXC banks, or

- Combine JPSI banks with GTRK banks for the same event to create BEXC banks.

MNBEXC monitors the type of $B$ created for each BEXC bank and WEBEXC is the routine that chooses the BEXC bank containing the best $B$ candidate for a given event. Finally, NTBEXC performs the same basic function as NTJPSI by preparing an ntuple in PAW. This outlines the general procedure for $\mathrm{B}$ selection from a strictly 'computer software' point of view. The succeeding couple of chapters explain the analysis from the physics standpoint. 


\section{Bibliography}

[3.1] E. Persico, E. Ferrari, and S. Segré, Principles of Particle Accelerators, 45,1968 .

[3.2] K. Meisner, et al., A Low Level RF System for the Fermilab Tevatron, IEEE PAC proceedings (1987)

[3.3] F. Abe, et al., The CDF Detector, Nucl. Instr. and Meth. A271 (1988) 387.

[3.4] G. Ascoli, et al., CDF Central Muon Detector, Nucl. Instr. and Meth. A268 (1988) 33.

[3.5] F. Bedeschi, et al., Design and Construction of the CDF Central Tracking Chamber, Nucl. Instr. and Meth. A268 (1988) 50.

[3.6] D. Amidei, et al., The CDF SVX: a Silicon Vertex Detector for a Hadron Collider, Nucl. Instr. and Meth. A289 (1990) 388.

[3.7] Rohm Technologies, Inc., 195 Canal Street, Malden, MA 02148 This company is responsible for its construction.

[3.8] D. Amidei, et al., A Two Level Fastbus Based Trigger System for CDF, Nucl. Instr. and Meth. A269 (1988) 51.

[3.9] E. Barsotti, et al., Fastbus Data Acquisition for $C D F$, Nucl. Instr. and Meth. A269 (1988) 82. 
[3.10] Marjorie Shapiro, David R. Quarrie, and Elizabeth Sexton. A Beginner's Guide to Analysis_Control and Build_Job, CDFNOTE 384

[3.11] René Brun, et al., PAW - Physics Analysis Workstation, CERN (1989). 


\section{Chapter 4}

\section{B Meson Reconstruction}

The goal is to find B mesons, which seems a simple one since there is sufficient ambient energy available to produce particles much heavier. However, they are not directly observed so the process for finding them is equivalent to a private investigator looking for clues in order to solve a case. The 'clues' are the daughter products left in the wake of the B decay. This chapter discusses the types of daughter products under scrutiny and their kinematic requirements typical for B decays.

\subsection{Decay Products}

\subsubsection{Dimuon Candidate Selection}

The previous chapter discussed the fact that the triggering processes which start the search for B candidates are the dimuon triggers associated with the CFT. Various requirements exist in this analysis to search for appropriate dimuon pairs. Valid muon are classified as any of the 4 types,

1. $\mathrm{CMU}$

2. CMP 


\section{CMUP}

4. $\mathrm{CMX}$

where CMUP implies that a muon has hits in both the CMU and CMP. Type 5 muons are also valid in which CMU and CMX hits exist for the same muon track, although the small geometrical overlap between the two sections makes this type rather rare. Two remaining types of muons involving both the CMP and CMX in any fashion are disallowed because of the non-overlapping coverage of the two sections. Individual muon transverse momenta are required to be above $1.5 \mathrm{GeV} / \mathrm{c}$. This is not surprising despite the fact that most of the dimuon events enter through the 2-2 trigger. The trigger has some 'turn on' efficiency associated with it in which a substantial number of muons below 2 $\mathrm{GeV} / \mathrm{c}$ pass the 2-2 trigger. Note that a specific trigger is not chosen for this part, or any other part for that matter, of this analysis which is equivalent to selecting all the triggers including the 3-1. This was done to increase the raw dimuon and resultant $\mathrm{J} / \psi$ sample size. The minimum $p_{T}$ cut was applied to both muon legs to mimic to some degree the symmetric nature of the 2-2 trigger since most of the dimuon events written to tape enter through this trigger.

\begin{tabular}{|l|c|}
\hline Description & Maximum Allow Value \\
\hline \hline Fit $\chi_{x}^{2}$ of CTC-CMU match & 9 \\
Fit $\chi_{z}^{2}$ of CTC-CMU match & 9 \\
Fit $\chi_{x}^{2}$ of CTC-CMP match & 9 \\
Fit $\chi_{x}^{2}$ of CTC-CMX match & 12 \\
Fit $\chi_{z}^{2}$ of CTC-CMX match & 12 \\
\hline
\end{tabular}

Table 4.1: CTC matching $\chi^{2}$ cuts to muon chamber sections.

To ensure that muons emanate from the beam collisions, not from outside the CDF detector, muon tracks in the CTC must correlate to an energy 
deposition in the hadron calorimeters which is greater than $.5 \mathrm{GeV}$. Standard procedure is to determine a set of quality cuts for the CTC tracks fitted to muon chamber hits. These are outlined in Table 4.1. Several combinations of muon chamber sections are permitted for dimuon pairs. The majority of the dimuon events are due to the following combinations in descending order,

- CMU-CMU (Most combinations)

- CMU-CMUP

- CMU-CMX

- CMUP-CMUP

- CMUP-CMX

- $\mathrm{CMX}-\mathrm{CMX}$

- CMU-CMP

- CMUP-CMP (Least combinations)

There exists a charge correlation cut of -1 for the two muons, which implies that they have opposite charge, and the mass window of the raw invariant mass of the pair must drop in the window between 2.8 and $3.4 \mathrm{GeV} / \mathrm{c}^{2}$. The $p_{T}$ of the pair must be above $1.5 \mathrm{GeV} / \mathrm{c}$. Figure 4.1 shows the breakdown of the candidates in this $0.6 \mathrm{GeV} / \mathrm{c}^{2}$ mass window for the 8 major muon chamber combinations.

Additional track quality requirements exist for all tracks, particularly those pertaining to the dimuon pair. A balance must be maintained between the demand for well measured tracks and the need to accept as many candidates as possible. Obviously, more hits in a specific detector section such as the CTC imply a better measured track. However, 'overmeasuring' tracks by requiring most or all layers to have hits, significantly reduces the statistics of the sample. For this analysis, a minimum number of 2 axial CTC superlayers 

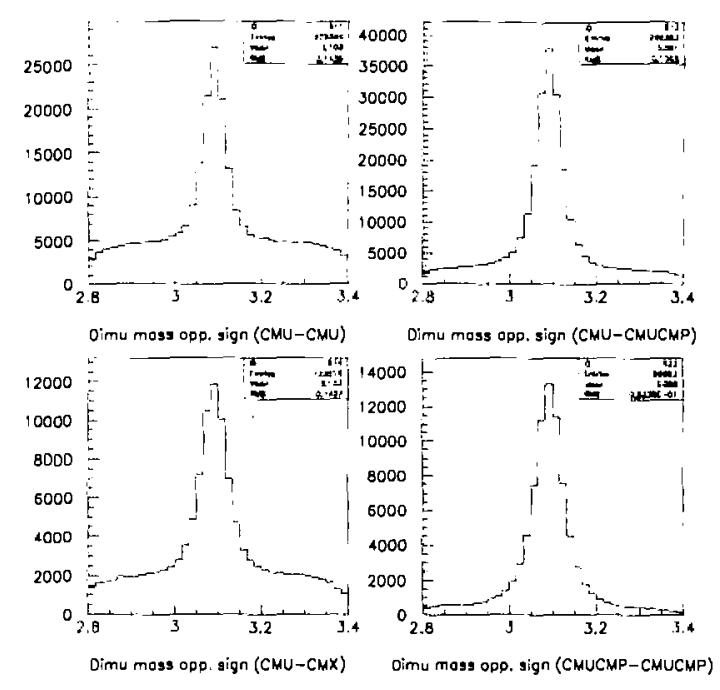

Figure 4.1: Raw invariant mass dimuon combinations for various CMU, CMP, and CMX permutations
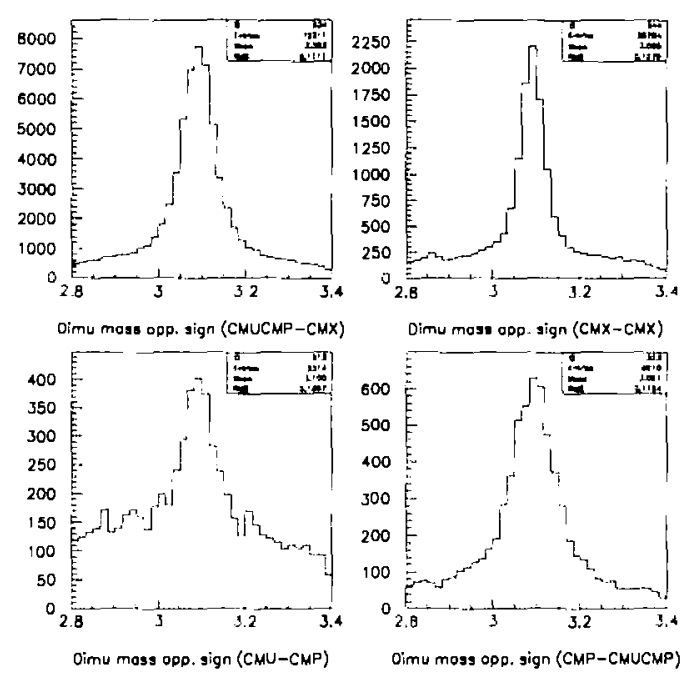
must have 5 hits each since most of the tracking in the CTC includes the axial superlayers and at least 2 CTC stereo superlayers must each have 2 hits. CTC tracks passing the two prior constraints and associated with any of the muon chamber systems must also have at least 2 SVX layer hits. This serves to better reconstruct the $\mathrm{J} / \psi$ vertex in coordinate space. The nomenclature commonly used for the track fits are CTC only and CTC/SVX. The preferred technique for measuring tracks is to take information from both the CTC and SVX, and for muon legs it is mandatory. A 2 hit SVX track with at least one shared hit is classified as a bad SVX track. A shared SVX hit is one in which more than one track traverses the same interstrip region of silicon strip detector. Although the cluster sharing within the SVX' has been extensively studied for application to the top search[4.1], it was felt that ignoring shared clusters was the safer approach rather than including them.

\subsection{2 $\Psi$ and $K_{s}^{o}$ Reconstructions}

The symbol $\Psi$ denotes the $\mathrm{J} / \psi$ or $\psi^{\prime}, \mathrm{J} / \psi$ candidates are selected that satisfy the following criteria. First, each of the oppositely charged muon legs from the $\mathrm{J} / \psi$ must be reconstructed within the $\operatorname{SVX}\left(\mathrm{SVX}^{\prime}\right)$, i.e. that the tracks are fitted using a combined CTC/SVX fit. Second, the transverse momentum of each muon is to be greater than $1.5 \mathrm{GeV} / \mathrm{c}$, a condition that already exists for the dimuon pair, but it is stated here to reemphasize the point. Third, both muons are constrained to come from a common vertex which is obviously the case for muons coming from real $\mathrm{J} / \psi$ 's. This last condition is referred to as a 'vertex constraint'. The invariant mass of the dimuon pair is recalculated since the vertex constraint requirement slightly alters the fitted track parameters. Figure 4.2 shows the mass distribution for the $\mathrm{J} / \psi$ 's. To be considered a candidate $\mathrm{J} / \psi$, the dimuon pair must reside in a mass window of $\pm 80 \mathrm{MeV} / \mathrm{c}^{2}$ (about $3 \sigma$ ) around the world average value. The value of $3 \sigma$ was determined by Olivier Snider and Alan Spies, et al., [4.2] by looking at the signal width for $\mathrm{J} / \psi$ 's with transverse momenta 
above $15 \mathrm{GeV} / \mathrm{c}$. The number of $\mathrm{J} / \psi$ candidates is $243722 \pm 562$ which is determined by a Gaussian fit to the peak distribution with a flat background and background is then subtracted from the signal region.

To increase statistics in both the B sectors, $\psi(2 \mathrm{~S})$ candidates are included. $\psi(2 S)$ events are reconstructed according to the decay $\psi(2 S) \rightarrow J / \psi \pi^{+} \pi^{-}$ The search for these candidates involves combining $\mathrm{J} / \psi$ events with two oppositely charged tracks different from those used to reconstruct the $\mathrm{J} / \psi$. Since CDF has no real particle identification other than muons, and electrons to a degree, tracks are assigned masses. After selecting all tracks in the event and assigning the desired masses to them, the best combination is chosen. This procedure is applied to the track pair associated with the $\psi(2 \mathrm{~S})$ in which the tracks are assigned charged pion masses. The invariant mass of the pion pair is required to be less than $600 \mathrm{MeV} / \mathrm{c}^{2}$ in order for the $J / \psi \pi^{+} \pi^{-}$to be in a reasonable $\psi(2 \mathrm{~S})$ mass range. The two muons from the $\mathrm{J} / \psi$ and the two pion tracks are fitted to a common vertex such that events with a fit $\chi^{2}$ probability below $1 \%$ are rejected. The combined $p_{T}$ of the four track system is required to be greater than $3 \mathrm{GeV} / \mathrm{c}$. Candidate $\psi(2 \mathrm{~S})$ events lie within a fairly narrow mass window of $\pm 20 \mathrm{MeV} / \mathrm{c}^{2}$ around the world average $\psi(2 \mathrm{~S})$ mass. From these cuts the number of $\psi(2 \mathrm{~S})$ candidates is $3577 \pm 97$. Figure 4.3 shows the $\psi(2 \mathrm{~S})$ mass distribution over the mass range from 3.6 to $3.8 \mathrm{GeV} / \mathrm{c}^{2}$. The distribution is fit for a Gaussian signal plus polynomial background and the number of background subtracted events is determined.

The search for $\psi(2 S) \rightarrow \mu^{+} \mu^{-}$was also performed. However, the statistics were exceedingly small with rather poor quality in the signal region so these were excluded from consideration.

Upon the reconstruction of a $\mathrm{J} / \psi$ or $\psi(2 \mathrm{~S})$ event, a subsequent search is initiated for $K_{s}^{\circ}$ candidates from the decay $K_{s}^{\circ} \rightarrow \pi^{+} \pi^{-}$. The search involves looking again for a pair of oppositely charged tracks that are assumed to be charged pions. Each pion must have an impact parameter whose absolute value exceeds $2 \sigma$, where $\sigma^{2}=\sigma_{\text {measured }}^{2}+\sigma_{\text {beam }}^{2}, \sigma_{\text {measured }}$ is the measurement error, and $\sigma_{\text {beam }}$ is the beam spot size. Both pions are also constrained 


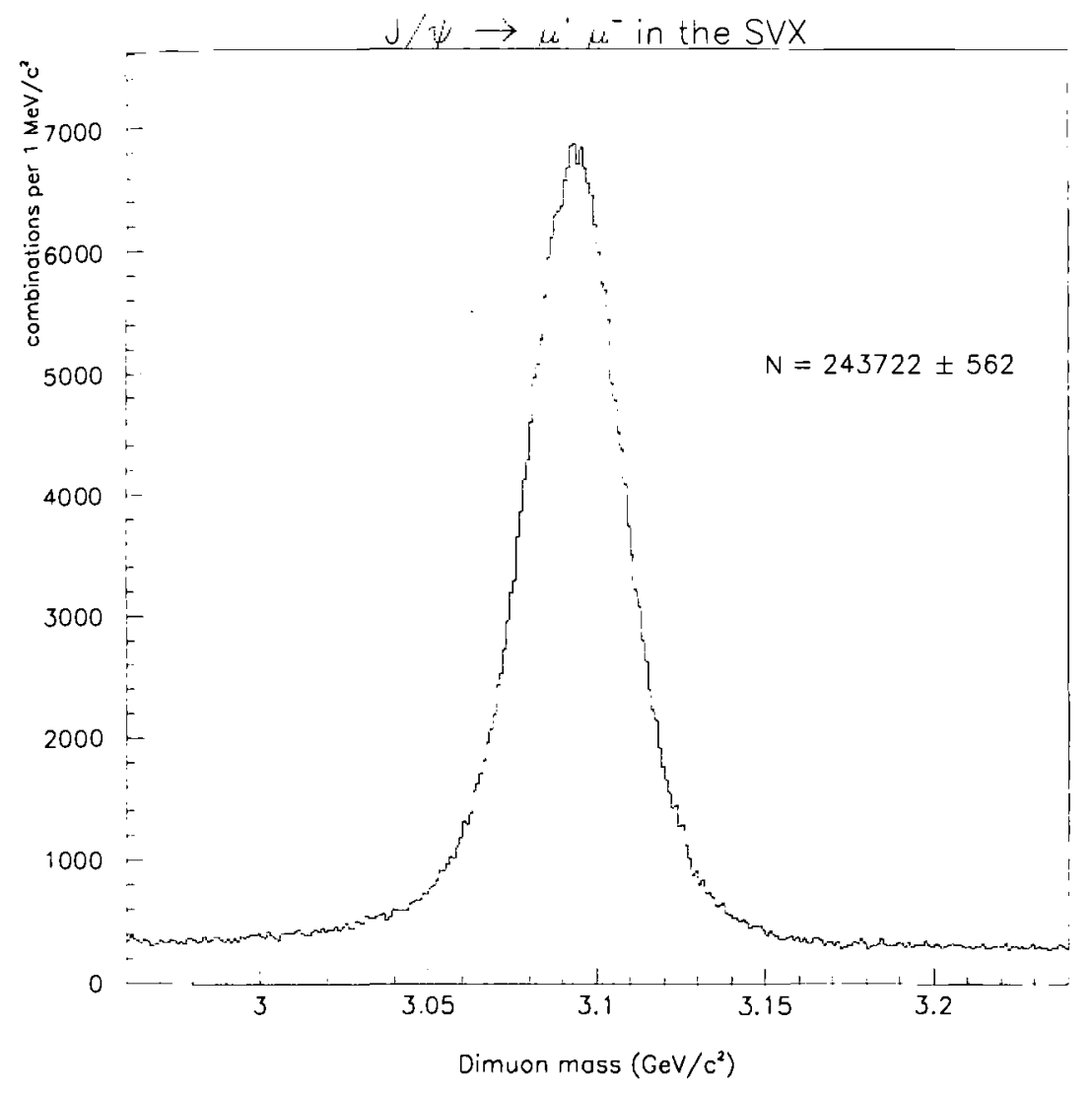

Figure 4.2: $\mathrm{J} / \psi$ mass distribution. The signal region is defined as a \pm 80 $\mathrm{MeV} / \mathrm{c}^{2}$ window about the world average value. The number quoted in the plot is after background subtraction in the signal region. 


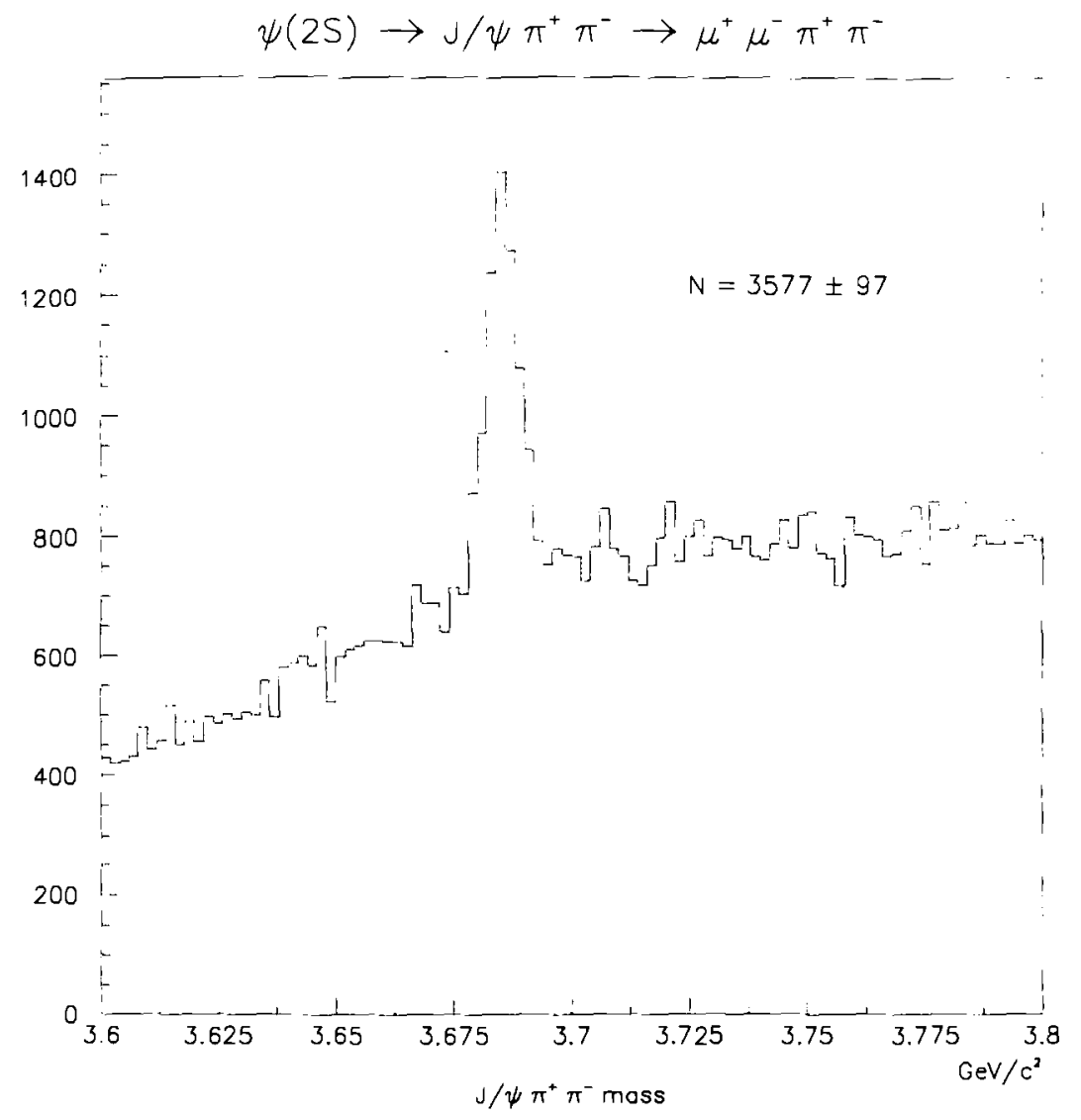

Figure 4.3: $\psi(2 \mathrm{~S})$ mass distribution. The signal region is defined as a \pm 20 $\mathrm{MeV} / \mathrm{c}^{2}$ window about the world average value. The number quoted in the plot is after the polynomial background subtraction to the Gaussian fit of the signal region. 


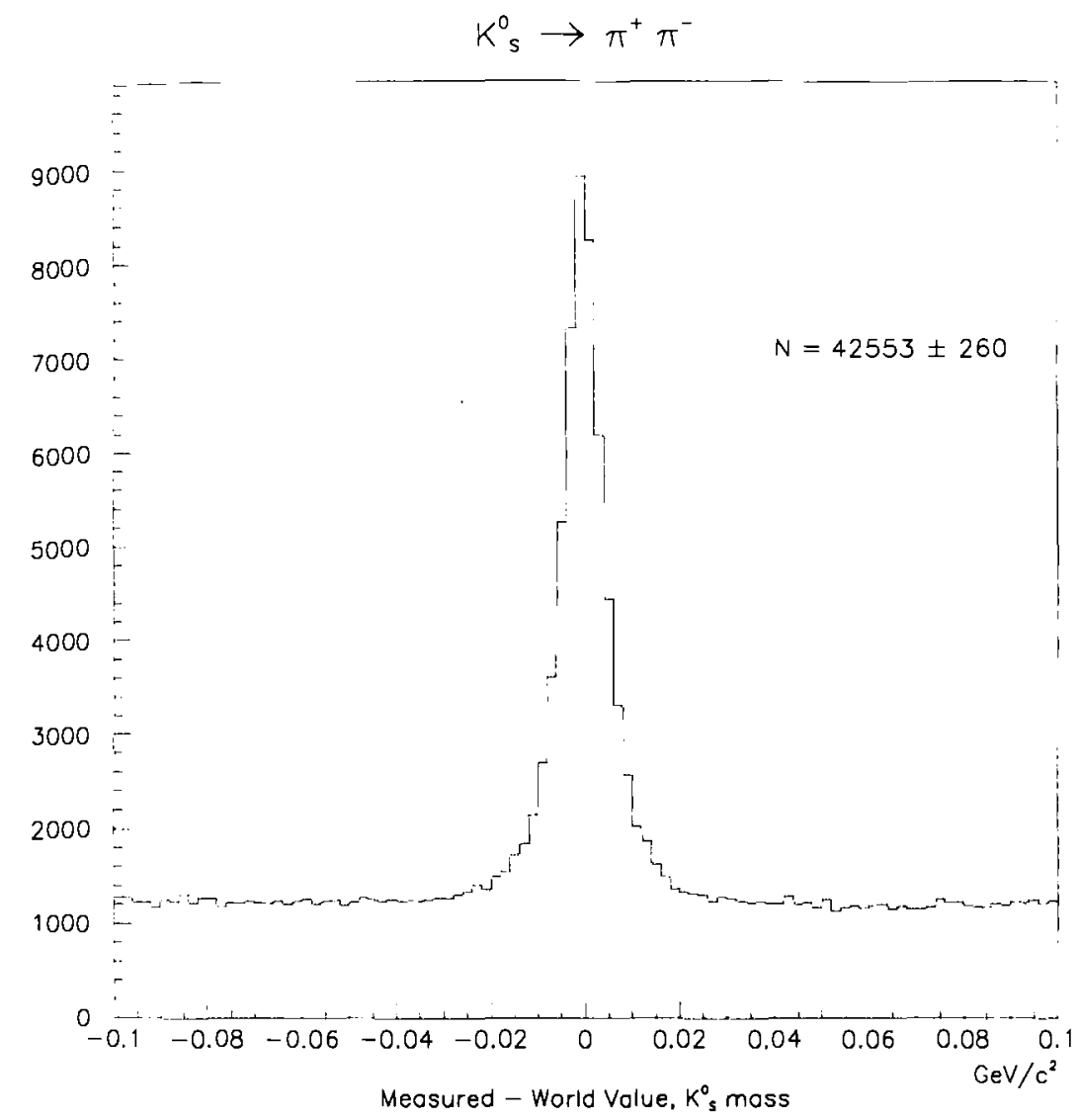

Figure 4.4: $\Delta K_{a}^{o}$ mass distribution. The histogram is the addition of the individual ones for the permutations of SVX/CTC and CTC only fits. The background subtracted number of events is found from a Gaussian fit to the signal with a flat background. The signal region is defined as a $\pm 20 \mathrm{MeV} / \mathrm{c}^{2}$ window about the world average value. 
to come from a common vertex with a fit $\chi^{2}$ probability above $1 \%$. The reconstructed $K_{s}^{o}$ is required to have a positive decay length with respect to the $\mathrm{J} / \psi$ vertex and the minimum distance between its flight path and the $\mathrm{J} / \psi$ vertex is $2 \mathrm{~mm}$ in the transverse plane. Viable $K_{s}^{\circ}$ candidates must lie within a $\pm 20 \mathrm{MeV} / \mathrm{c}^{2}$ mass window around the world average value. Figure 4.4 shows the observed $K_{s}^{o}$ mass peak subtracted from the world value of $497.7 \mathrm{MeV} / \mathrm{c}^{2}$ summed over the three types of fit combinations between the two tracks outlined in Table 4.2.

\begin{tabular}{|l|l|c|}
\hline \hline Track 1 Fit & Track 2 Fit & \# Background Subtracted Events \\
\hline CTC only & CTC only & $7680 \pm 98$ \\
SVX/CTC & SVX/CTC & $28163 \pm 214$ \\
CTC only & SVX/CTC & $7029 \pm 106$ \\
\hline \hline
\end{tabular}

Table 4.2: Breakdown of the 2 track fit combinations used in $K_{s}^{\circ}$ reconstruction.

\subsection{3 $\mathrm{B}_{u}, \mathrm{~B}_{d}$, and $\mathrm{B}_{s}$ Mesons}

The two primary searches that prompt this analysis remain the $\mathrm{B}_{\mathbf{u}}$ and $\mathrm{B}_{d}$ mesons. The $\mathrm{B}$, is included because of the collaborative work done by the author in support of a colleague[4.3].

$B_{u}$ mesons are reconstructed from the following decay modes;

- $\mathrm{J} / \psi K^{+}$

- $\mathrm{J} / \psi \mathrm{K}^{*}(892)^{+}$

- $\psi(2 S) K^{+}$

- $\psi(2 \mathrm{~S}) \mathrm{K}^{*}(892)^{+}$

and the conjugate is implied for the case of the $B^{-}$. In all cases the $\mathrm{J} / \psi$ and $\psi(2 \mathrm{~S})$ are mass constrained to their respective values. A single track search is then performed and the track is assigned the charged kaon mass. All tracks 
from $\mathrm{J} / \psi K^{+}$or $\psi(2 \mathrm{~S}) K^{+}$are vertex constrained. For the $\mathrm{K}^{*}(892)^{+}, K_{s}^{\circ}$ candidates are paired with an additional track assumed to be a charged pion. Tracks from the $K_{s}^{o}$ are vertex constrained but not to the eventual B vertex since the $K_{s}^{o}$ travels a fair distance before it decays. Instead, the $K_{s}^{o}$ itself is required to point back to the $\mathrm{B}$ vertex. $K_{s}^{o} \pi^{+}$combinations are stipulated to be within a $\pm 80 \mathrm{MeV} / \mathrm{c}^{2}$ invariant mass window around the world average $\mathrm{K}^{*}(892)^{+}$mass of $891.59 \mathrm{MeV} / \mathrm{c}^{2}$.

$B^{\circ}$ mesons are also reconstructed from decays analogous to the $B^{+}$;

- $\mathrm{J} / \psi K_{s}^{o}$

- $\mathrm{J} / \psi \mathrm{K}^{*}(892)^{\circ}$

- $\psi(2 \mathrm{~S}) K_{0}^{\circ}$

- $\psi(2 \mathrm{~S}) \mathrm{K}^{*}(892)^{\circ}$

in which the conjugate is also implied for the $\overline{B^{\circ}}$. Once again the $\Psi$ is mass constrained. Combinations of $\mathrm{J} / \psi$ or $\psi(2 \mathrm{~S})$ are matched with $K_{\text {s }}^{o}$ candidates. The two pion tracks emanating from the $K_{g}^{o}$ are vertex constrained again, with the requirement that the $K_{0}^{o}$ must point to the $\mathrm{B}$ vertex. For the remaining two cases involving the $\mathrm{K}^{*}(892)^{\circ}$, a search is conducted for two oppositely charged tracks to combine with a $\Psi$ candidate. The tracks are assumed to be a $K \pi$ combination and all tracks are vertex constrained. Early in the analysis of Run 1B data, significant degradation of the $B_{d}$ signal region was observed. To bring the sample purity to more tolerable levels, the transverse momenta of the charged kaon and pion were required to be greater than 1 and $.5 \mathrm{GeV} / \mathrm{c}$, respectively. The mass window for $\mathrm{K}^{*}(892)^{\circ}$ acceptance is $\pm 80 \mathrm{MeV} / \mathrm{c}^{2}$ around the world average value of $896.10 \mathrm{MeV} / \mathrm{c}^{2}$.

$B$, mesons are reconstructed from two decay modes;

- $\mathrm{J} / \psi \phi$

- $\psi(2 S) \phi$. 
The procedure for finding $B$, candidates is virtually identical to that of the $B_{d} \rightarrow \Psi K^{*}(892)^{\circ}$ decay. A search is performed for two oppositely charged tracks. The tracks are assumed to be charged kaons which are combined to form $\phi$ candidates. The $\Psi$ is mass constrained, and all tracks are constrained to come from a common vertex. The $\phi$ is a fairly narrow resonance such that $M_{\text {inv }}\left(K^{+} K^{-}\right)<10 \mathrm{MeV} / \mathrm{c}^{2}$ around world average $\phi$ value of 1.019413 $\mathrm{GeV} / \mathrm{c}^{2}$.

A natural drawback to the lack of specific particle identification is that the mass assignment to tracks is somewhat arbitrary. The most obvious example concerns the case of the $K^{*}(892)^{\circ}$. The mass assignments to the kaon and pion must take into account that the order in which tracks are assigned matters. If track 1 is linked to a kaon then track 2 must be linked to a pion. However, the transposition of mass assignments is also considered as a potential candidate for the same 2 tracks. This duplication process is a necessary hazard that needs clarification in order to prevent biasing the lifetime by introducing multiple candidates for the same tracks. In removing duplicate events, one must choose not only one set of mass assignments to the track pair, but the best one in a fashion that does not introduce some new systematic bias in the process.

The process of removing duplicate events reiterated here was first outlined in reference 4.1 by Olivier Snider and Alan Spies. The basic philosophy of how to remove duplicate events is one of common sense: Real candidates should pass some stiffer kinematic cuts, but more importantly, they should form better $\mathrm{B}$ vertices. Transverse momenta of the $B_{i}$ mesons are required to be greater than $6 \mathrm{GeV} / c$ where $i \equiv u, d, s$. An additional requirement exists for the daughter kaons such that $p_{T}>1.25 \mathrm{GeV} / \mathrm{c}$ for the $K^{+}, K_{s}^{\circ}$, $K^{*}(892)^{+}$, and $K^{*}(892)^{\circ}$. The $p_{T}$ of the $\phi$ must also be above $1.25 \mathrm{GeV} / \mathrm{c}$ although the problem of track mass assignments does not exist since both tracks from the $\phi$ are assigned charged kaon masses. These cuts provide the 8mallest statistical error on the background subtracted signal and are the first filters in the duplicate removal process. 
The next filter deals with removing duplicate $B$ events arising from the $\mathrm{K} \pi$ mass assignment ambiguity for the $K^{*}(892)^{\circ}$. If this is the only difference between multiple $B$ candidates, then to differentiate the fit $\chi^{2}$ probabilities of these multiple candidates is not applicable, because the same two tracks are considered. The fit $\chi^{2}$ is a function of the 5 track parameters and is indifferent to mass assignment. Therefore, the choice of $B$ meson is determined by the mass assignment resulting in a value closest to the world $K^{*}(892)^{\circ}$ value.

The remaining duplicate $B$ candidates are distinguished by their vertex fit quality. The track combination with the best fit $\chi^{2}$ to a secondary vertex is chosen for a given event.

The resultant mass distributions for the charged, neutral, and strange $\mathrm{B}$ mesons are plotted in Figures 4.5, 4.6, and 4.7. The vertical axes are the measured $B_{u}$ mass - world value of $5.2786 \mathrm{GeV} / \mathrm{c}^{2}$ measured $B_{d}$ mass world value of $5.2786 \mathrm{GeV} / \mathrm{c}^{2}$, and the measured $B$, mass - world value of $5.3630 \mathrm{GeV} / \mathrm{c}^{2}$. The histogram ranges are plotted $240 \mathrm{MeV} / \mathrm{c}^{2}$ wide centered at 0 . The signal regions are defined as windows that extend $\pm 30 \mathrm{MeV} / \mathrm{c}^{2}$ around their respective world average $B$ meson masses. As seen in Figures 4.5 through 4.7 the mass plots shift these world average values to the origin. The upper and lower sidebands windows for the $B_{u}$ and $B_{d}$ are each 60 $\mathrm{MeV} / \mathrm{c}^{2}$ wide and start at $\mp 60 \mathrm{MeV} / \mathrm{c}^{2}$ and extend to $\mp 120 \mathrm{MeV} / \mathrm{c}^{2}$ from the world average value. The sidebands for the $B$, also start at $\mp 60 \mathrm{MeV} / \mathrm{c}^{2}$ but extend $\mp 200 \mathrm{MeV} / \mathrm{c}^{2}$. The reason for the larger $B$, sideband regions is that the number of sideband events is too small to properly fit the events for a smaller window. There exist two $30 \mathrm{MeV} / \mathrm{c}^{2}$ gaps that separate signal from lower and upper sideband regions. The reasoning for these gaps is left for the discussion concerning the lifetime calculation in the next chapter. The total numbers of background subtracted events for the $B_{u}, B_{d}$, and $B_{s}$ with c $\tau>100 \mu \mathrm{m}$ are 671,357 , and 52 events, respectively.

Figure 4.8 shows the duplicates that were removed for the two high statistics decay channels of the $B_{u}$ and $B_{d}$ sectors. The Run $1 \mathrm{~A}$ analysis by Alan Spies and Olivier Snider shows a higher rate of duplicates for the $B_{d}[4.4]$. 
RUN $1 A+1 B$ CDF PRELIMINARY

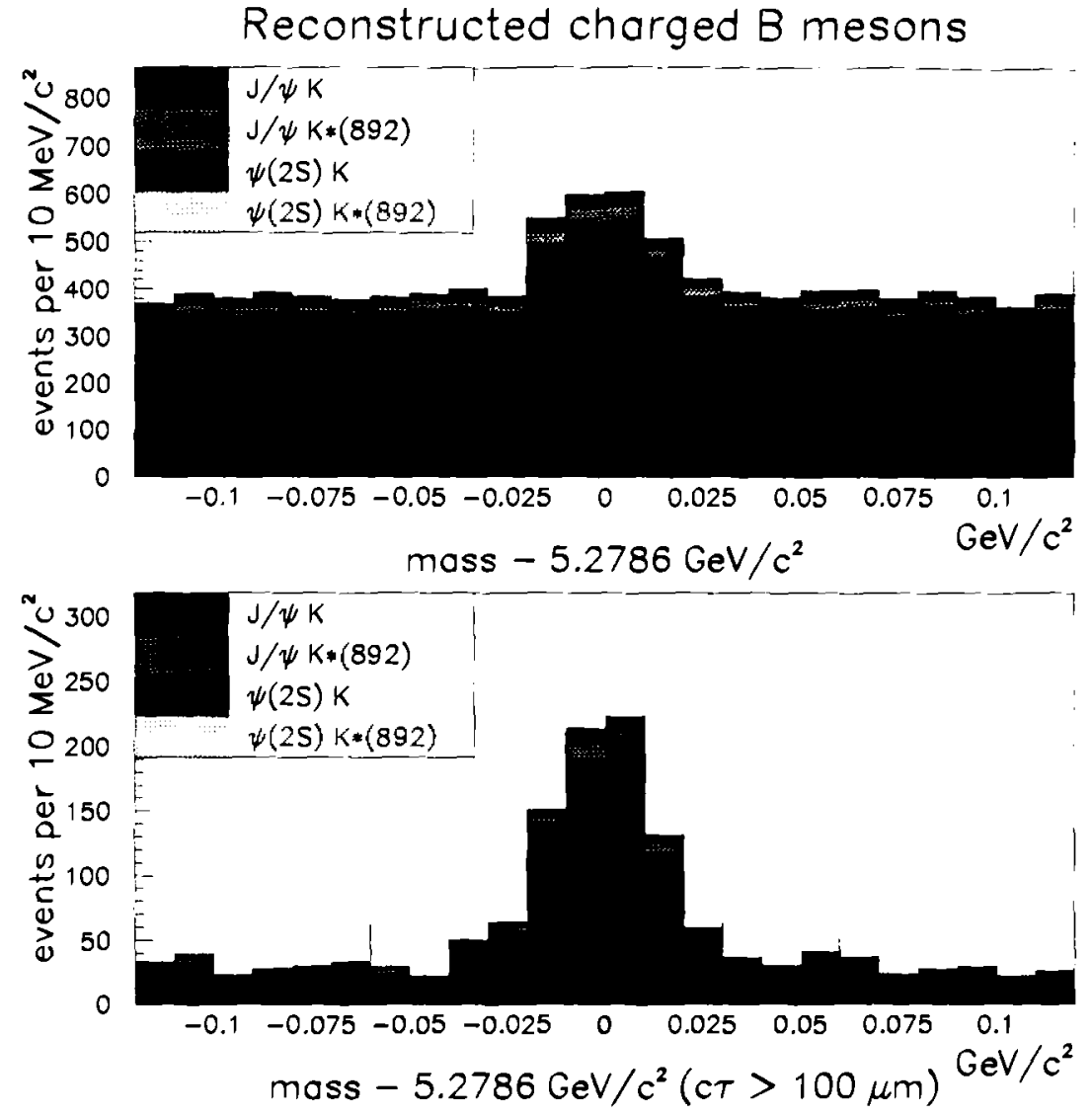

Figure 4.5: $B_{u}$ mass distributions for $p_{T}\left(B_{u}\right)>6 \mathrm{GeV} / \mathrm{c}$ and $p_{T}\left(K_{j}\right)>1.25$ $\mathrm{GeV} / \mathrm{c}$ where the index $j$ refers to a $K^{+}$or $K^{*}(892)^{+}$meson. The lower distribution has an additional cut of $\mathrm{c} \tau>100 \mu \mathrm{m}$. The various shades of color indicate the contributions of the 4 major decay channels. 


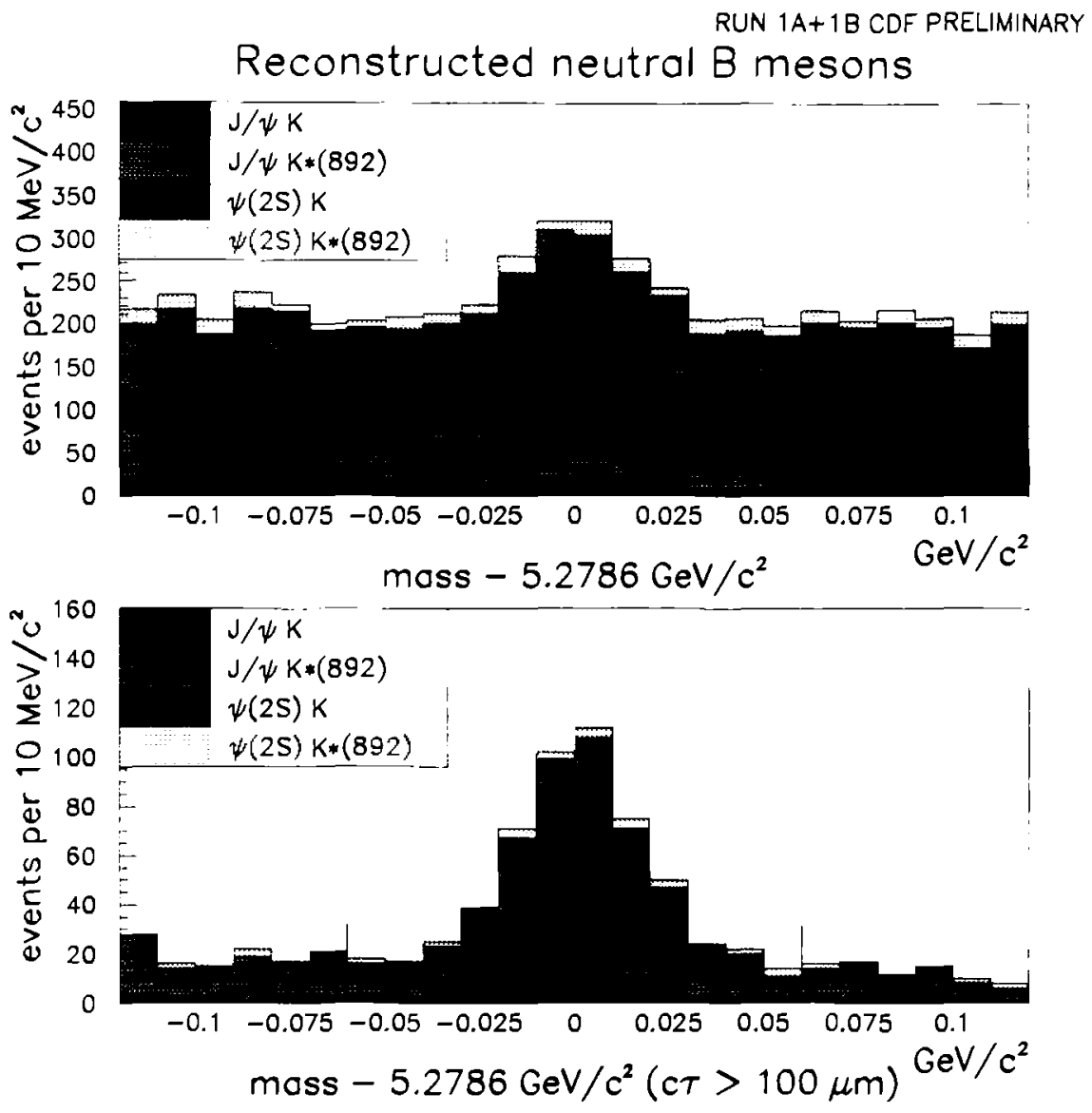

Figure 4.6: $B_{d}$ mass distributions for $p_{T}\left(B_{d}\right)>6 \mathrm{GeV} / \mathrm{c}$ and $p_{T}\left(K_{m}\right)>1.25$ $\mathrm{GeV} / \mathrm{c}$. The index $m$ refers to the $K_{a}^{o}$ or $K^{*}(892)^{\circ}$. The lower distribution has an additional cut of $\mathrm{c} \tau>100 \mu \mathrm{m}$. Significant contribution from the $K_{s}^{o}$ are more prevalent with the addition of almost $90 \mathrm{pb}^{-1}$ of Run $1 \mathrm{~B}$ data to Run 1A. 


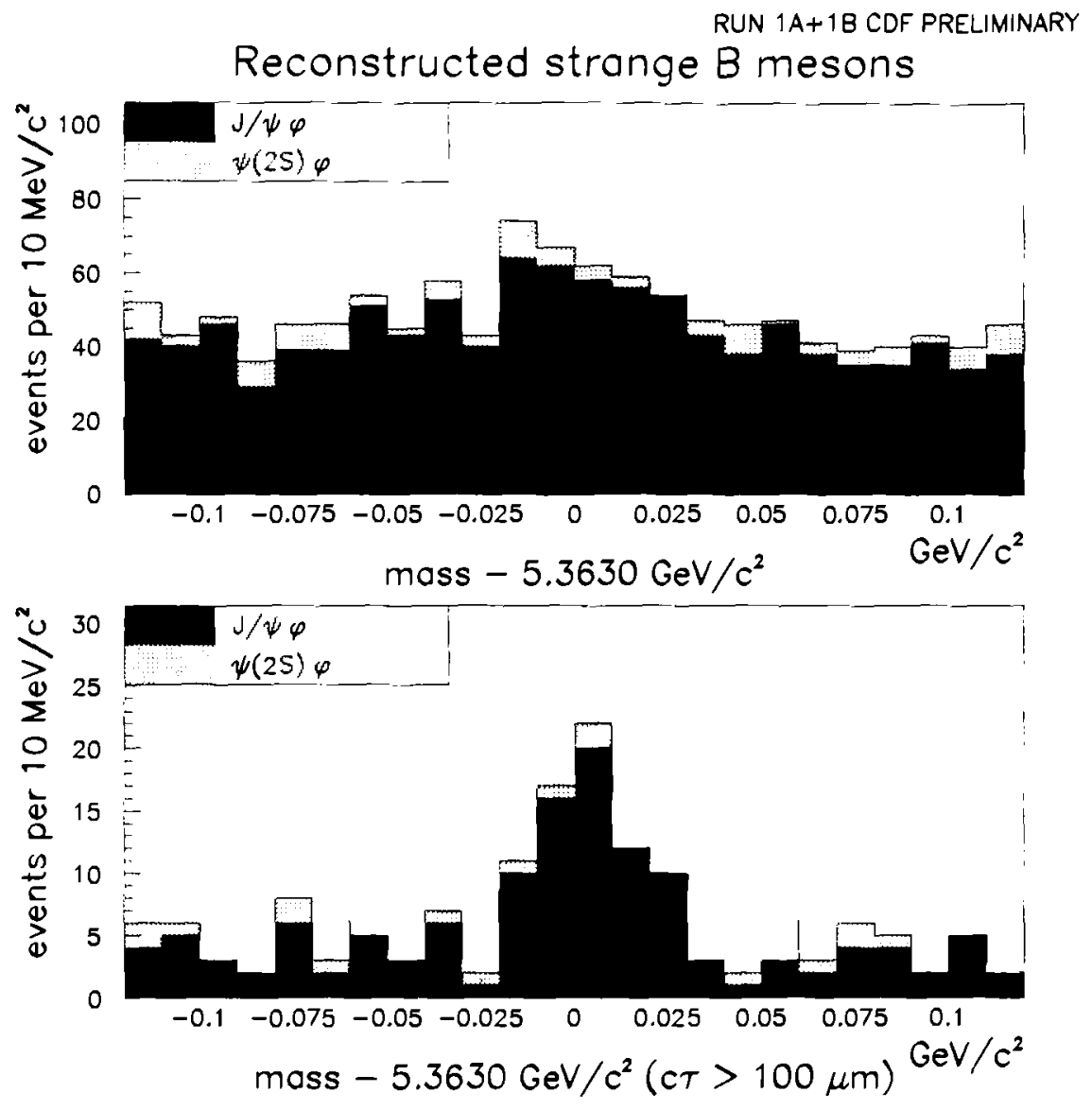

Figure 4.7: $B_{s}$ mass distributions for $p_{T}\left(B_{s}\right)>6 \mathrm{GeV} / \mathrm{c}$ and $p_{T}(\phi)>1.25$ $\mathrm{GeV} / \mathrm{c}$. The lower distribution has an additional cut of $\mathrm{c} \tau>100 \mu \mathrm{m}$. 
The major reason for a lower duplicate event rate in this latest analysis lies in the stiffer transverse momentum cuts for the $K^{*}(892)^{\circ}$ decay products. The cuts of $p_{T}\left(K^{ \pm}\right)>1 \mathrm{GeV} / \mathrm{c}$ and $p_{T}\left(\pi^{\mp}\right)>0.5 \mathrm{GeV} / \mathrm{c}$ help to distinguish candidates with higher fidelity by differentiating lower $p_{T} \mathrm{~K} \pi$ combinations. The distributions with or without a $100 \mu \mathrm{m}$ cut on $\mathrm{c} \tau$ indicate no preferred mass region for duplicate event removal for the $B_{u}$. For the $B_{d}$ this is not necessarily the case for decays involving the $K^{*}(892)^{\circ}$ since the second filtering step specifically targets the $K^{*}(892)^{\circ}$ events closest to its world average value. This preferential treatment does not bias the lifetime since the same two tracks are used and therefore, the lifetime of the B candidate is the same regardless of mass assignment. Figure 4.9 shows the same mass distributions with a cut on $\mathrm{c} \tau>100 \mu \mathrm{m}$. The enhancement of the signal region for $B_{d} \rightarrow J / \psi K^{*}(892)^{\circ}$ duplicates is more evident with this additional $\mathrm{c} \tau$ restriction.

Figure 4.10 illustrates the frequency of events over the course of Run $1 \mathrm{~A}$ and Run $1 \mathrm{~B}$ after the duplicate event removal procedures are applied. The luminosities for Run $1 \mathrm{~A}$ and Run $1 \mathrm{~B}$ are $20 \mathrm{pb}^{-1}$ and $90 \mathrm{pb}^{-1}$. The large gap between runs represents the replacement time taken to install the new silicon vertex detector. 

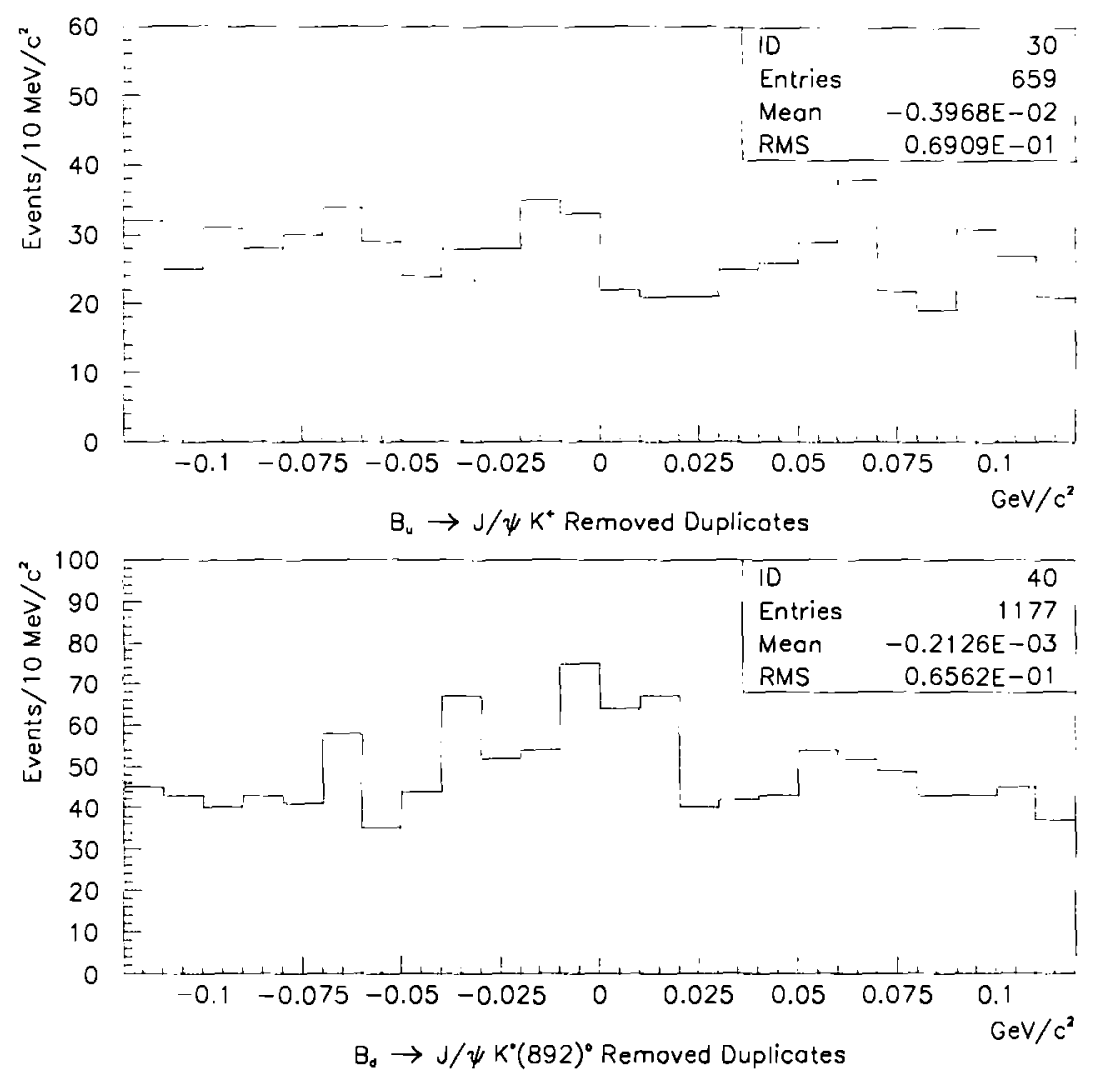

Figure 4.8: Removed duplicate events for the highest statistics decay channels of the $B_{u}$ and $B_{d}$. The horizontal axes for both plots are the measured - world value for the $B$ mass. 



Figure 4.9: Removed duplicate events for the highest statistics decay channels of the $B_{u}$ and $B_{d}$ with the additional requirement that $\mathrm{c} \tau>100 \mu \mathrm{m}$. The horizontal axes for both plots are the measured - world value for the $B$ mass. 

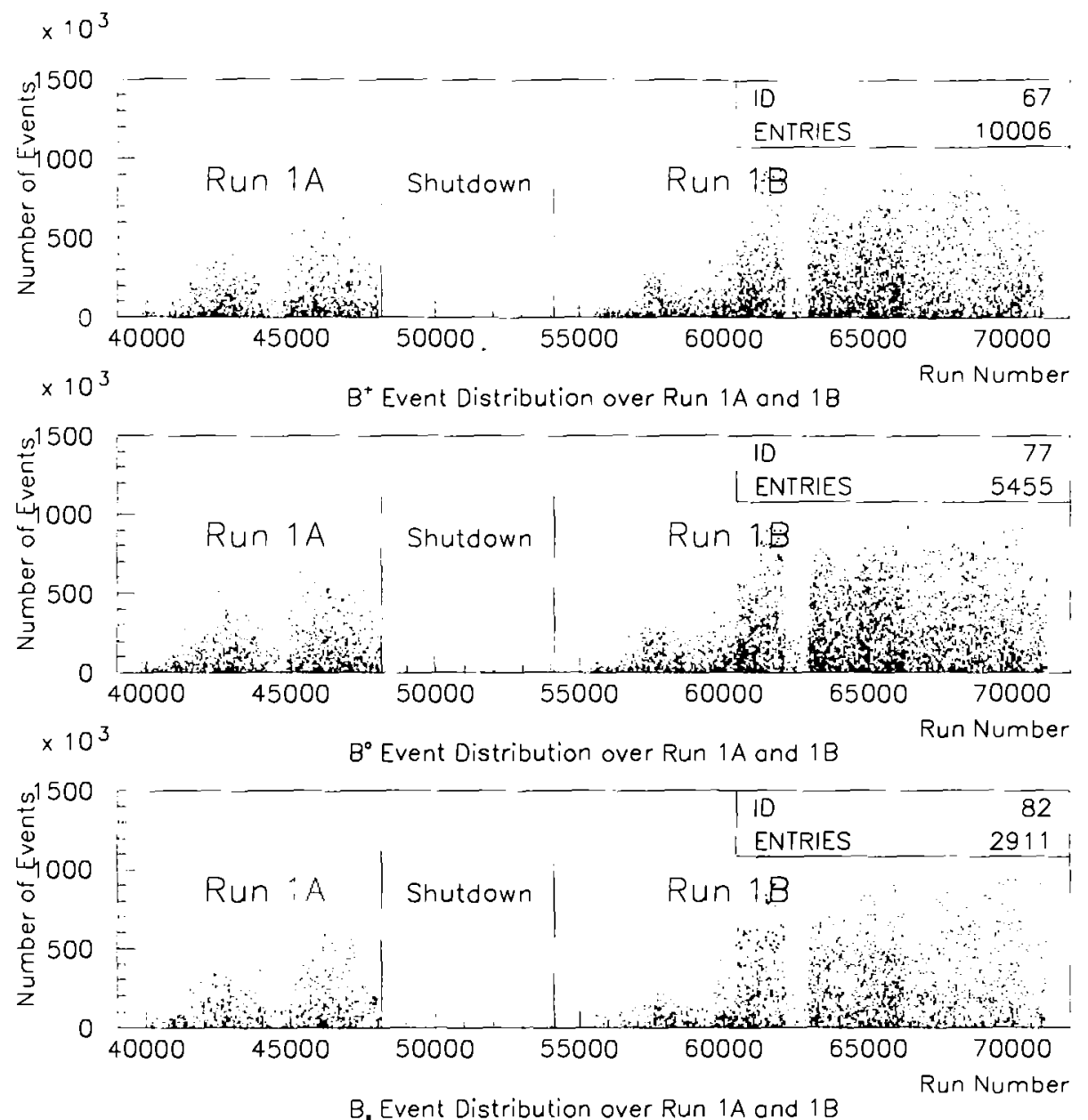

Figure 4.10: The event distribution for $\mathrm{B}$ candidates in all three sectors after duplicate removal procedures are applied. The long vertical lines indicate the shutdown period in which the SVX was replaced with the SVX' 


\section{Bibliography}

[4.1] H. Y. Chao, et al., The SVX' MC Simulation, CDF internal document number 2946.

[4.2] Olivier Snider and Alan Spies, et al., A Measurement of $B^{+}$and $B^{\circ}$ Lifetimes Using Exclusive Decay Channels, CDF internal document number 2345 .

[4.3] The $B$, exclusive lifetime was independently determined by myself and Farrukh Azfar. Being Farrukh's thesis topic my analysis served to support his lifetime measurement. Since a substantial portion of my time was spent in helping him, $B_{s}$ is included here so as to document the time spent.

[4.4] Alan Spies, A Measurement of the Charged and Neutral B Meson Lifetimes Using Fully Reconstructed Decay Modes, Ph.D. Thesis. 1994. 


\section{Chapter 5}

\section{Lifetime Analysis}

A precise measurement of the lifetime is dependent on the signal quality determined in the previous chapter. Once a reasonable $B$ mass peak is obtained, the proper decay length of the candidates is determined. The next two sections outline the calculation of $\mathrm{c} \tau$ for individual candidates and the fitting procedure used to determine the overall lifetimes of the B samples. The emphasis is on the $B_{u}$ and $B_{d}$ lifetimes.

\subsection{Calculation of $\mathrm{c} \tau$}

The determination of the proper decay length is straightforward. The proper time interval is given by,

$$
\tau=\sqrt{x^{\mu} x_{\mu}}
$$

using a metric tensor of the form

$$
\eta^{\mu \nu}=\left[\begin{array}{cccc}
1 & 0 & 0 & 0 \\
0 & -1 & 0 & 0 \\
0 & 0 & -1 & 0 \\
0 & 0 & 0 & -1
\end{array}\right]
$$


so that

$$
\tau=\sqrt{t^{2}-x^{2}-y^{2}-z^{2}}=t / \gamma .
$$

Since $t$ is the time interval in the lab frame, it is simply given by

$$
t=\vec{x} / \vec{v}=\frac{\vec{x} m_{B}}{m_{B} \vec{v}} .
$$

The expression for $\mathrm{c} \tau$ becomes,

$$
c \tau=\frac{\vec{x} m_{B} c}{m_{B} \vec{v} \gamma}=m_{B} c \frac{\vec{x} \cdot \vec{p}}{\vec{p}^{2}} .
$$

In the transverse plane $\vec{p} \rightarrow \overrightarrow{p_{T}}$ and the final form is reexpressed in terms of the quantity $L_{x y} \equiv \vec{x} \cdot \overrightarrow{p_{T}} /\left|\overrightarrow{p_{T}}\right|$,

$$
c \tau=\frac{L_{x y} m_{B} c}{\left|\vec{p}_{T}^{B}\right|} .
$$

The symbol, $m_{B}$, is the world average $B_{u}, B_{d}$, or $B_{s}$ mass and $\vec{p}_{T}^{B}$ is the measured transverse momentum vector of the given $B$ meson candidate. The distance, $L_{x y}$, is taken as the projected distance on the transverse plane between the primary and secondary vertices. $B$ candidates with sufficiently large magnitudes for $\vec{x}$ but negative values for $L_{x y}$ are classified as background events. The error on $c \tau$ is predominantly a function of the error on $L_{x y}$. The form for the error on $L_{x y}$ is given by,

$$
\sigma_{L_{\mathrm{xy}}}^{2}=\frac{1}{\overrightarrow{\vec{p}_{T}^{2}}} \mathbf{P}^{-1}\left(\sigma_{\text {beam }}^{2}+\sigma_{\text {Bvertex }}^{2}\right) \mathbf{P}
$$

where,

$$
\begin{gathered}
\mathbf{P}=\left[\begin{array}{l}
p_{x} \\
p_{y}
\end{array}\right] \\
\sigma_{\text {beam }}^{2}=\left[\begin{array}{cc}
\sigma_{x, \text { beam }}^{2} & 0 \\
0 & \sigma_{y, \text { beam }}^{2}
\end{array}\right]
\end{gathered}
$$




$$
\sigma_{\text {Bvertex }}^{2}=\left[\begin{array}{cc}
\sigma_{x, B v e r t e x}^{2} & \sigma_{x, B v e r t e x} \sigma_{y, B v e r t e x} \\
\sigma_{x, B v e r t e x} \sigma_{y, B v e r t e x} & \sigma_{y, B v e r t e x}^{2}
\end{array}\right]
$$

The momentum components are of the B meson. The $\sigma$ 's associated with the beam are the transverse components of the actual spot size. The beam does move slightly in the transverse direction during the course of a a run to give associated $\mathrm{x}$ and $\mathrm{y}$ errors. This will be included in the discussion of systematic errors.

The beam profile for Run 1A is circular with an average spot size of $36 \mu \mathrm{m}$ in the transverse plane. For Run $1 B$ the beam spot is almost circular with projections in the $\mathrm{x}$ and $\mathrm{y}$ directions of $25 \mu \mathrm{m}$ and $28 \mu \mathrm{m}$. The $z_{\circ}$ position is approximately Gaussian with a width, $\sigma_{z_{0}}$ close to $30 \mathrm{~cm}$. The primary vertex is obtained from SVX information stored in a database from files, if available, with the name SVX*.BPO or from the SVBP bank contained within the reprocessed $7.12 \mathrm{~J} / \psi$ data[5.1,5.2]. The primary vertex information is taken as the run averaged beam position as opposed to an event-by-event basis within a given run. This is slightly different in procedure than the earlier versions of this analysis which took the beam line from the VTVZ bank. The VTVZ bank contained beam line information from tracks measured within the CTC. The advantage of the SVBP bank is that the routine responsible for creating the bank uses an iterative fit of the 3-dimensional impact parameter $D\left(\phi_{\circ}, Z_{\circ}\right)$ using 4 hit SVX tracks. This makes it more precise and also faster since each track equates to a point in the fit. The 3-dimensional impact parameter is parameterized in terms of the following four quantities:

- Beam $X$ at $Z=0$,

- Beam $Y$ at $Z=0$,

- $\mathrm{dX} / \mathrm{dZ}$ and,

- $\mathrm{dY} / \mathrm{dZ}$. 
Starting values are input from the database and the iterative fit is performed to determine new values. In rare cases where there is no beam line information available from either the absence of $\mathrm{SVX}^{*}$. BPO files in the database or the lack of an SVBP bank then the event search routines restart until beam line information is found. This is shown in Chapter 3, Figure 3.17 in which the routine MNPVTX performs this function. Such is the case for small runs or other runs which are not considered 'good'. For example, 'bad' runs are considered to be low luminosity runs or runs in which parts of the detector were known to be inoperative or malfunctioning. In the end it makes little difference since only good runs contribute to the lifetime analysis.

\subsection{Lifetime Fitting}

Once the $\mathrm{c} \tau$ distributions are obtained for each $\mathrm{B}$ sector, the task remains to extract their lifetimes. The basic technique is similar to the one described in Reference 4.3 but several changes were necessary due to the increased sample size. The probability density functions are the well spring from which this technique flows, so the PDF's are derived after listing some fundamental assumptions which motivate their forms.

The peak region is assumed to contain both signal and background events such that for a true $\mathrm{B}$ meson candidate falling in this region, its proper decay length has a probability density that is a convolution of an exponential function plus a completely Gaussian resolution function. The measurement error on the proper decay length is equal to its Gaussian resolution. The background events in the peak region are assumed to have the same $c \tau$ distribution as events in the sideband regions. Thus, the background resolution function in this region has the same Gaussian form as the sideband function. Two exponential tails model the non-Gaussian portions of the sideband and background distributions. An additional positive exponential tail accounts for the existence of long lived events in the sidebands. The background shape 
of the mass distribution is assumed to be linear with symmetric sidebands around the $B$ mass as described in Chapter 4.

In the ideal world (a perfect detector, etc) the distribution for $\mathrm{B}$ mesons in the peak region would be seen as a $\delta$ function plus a single high side exponential tail. The $\delta$ function represents the prompt background component while the exponential tail accounts for the decay law associated with the $B$. Since this is not a perfect world, the actual distributions tend to be smeared by a predominantly Gaussian function which is most evident for the prompt component of the distribution. The peak region is a sum of Noise (background) and Signal probability density functions

$$
F_{\text {peak_region }}(x)=N(x)+S(x)
$$

in which the ideal scenario gives,

$$
F_{\text {peak }}(x)=\delta(x)+S(x)
$$

where $\mathrm{S}(\mathrm{x})$ contains the actual physics of the B decay,

$$
S(x)=\left\{\begin{array}{cl}
e^{-x / \lambda} / \lambda & \mathrm{x} \geq 0 \\
0 & \text { otherwise. }
\end{array}\right.
$$

The actual observed peak probability density function has the form

$$
F_{\text {peak }}(x)=N(x)+\int_{-\infty}^{+\infty} S\left(x^{\prime}\right) \mathcal{R}\left(x-x^{\prime}\right) d x^{\prime}
$$

in which,

$$
\begin{gathered}
\mathcal{R}\left(x-x^{\prime}\right)=\beta_{i} R_{\text {Gaussian }}\left(x-x^{\prime}\right)+\left(1-\beta_{i}\right) R_{\text {tails }}\left(x-x^{\prime}\right) \\
R_{\text {Gaussian }}\left(x-x^{\prime}\right)=e^{-z^{2} / 2} / \sqrt{2 \pi \sigma_{i}^{2}} \\
R_{\text {tails }}\left(x-x^{\prime}\right)=e^{-|z| \sigma_{i} / \mu_{i}} / 2 \mu_{i}
\end{gathered}
$$

and,

$$
z=\left(x-x^{\prime}\right) / \sigma_{i}
$$


The form for the background PDF is taken directly from the form of the sideband candidates, $F_{\text {sidebands }}(\mathrm{x})$,

$$
N(x)=F_{\text {sidebands }}(x)= \begin{cases}A \mathcal{R}(x)+\frac{f_{1}^{+}}{\lambda_{1}} e^{-x / \lambda_{1}}+\frac{f_{2}}{\lambda_{2}} e^{-x / \lambda_{2}} & \text { for } x>0 \\ A \mathcal{R}(x)+\frac{f_{1}^{-}}{\lambda_{1}} e^{x / \lambda_{1}} & \text { for } x<0\end{cases}
$$

where $A=\frac{1-f_{2}^{-}-f_{2}^{+}-f_{2}}{\sigma_{i} \sqrt{2 \pi}}$. The integration for the first part of the peak PDF gives,

$$
\beta_{i} \int_{-\infty}^{+\infty} S\left(x^{\prime}\right) R_{G a u s s i a n}\left(x-x^{\prime}\right) d x^{\prime}=\frac{\beta_{i}}{\lambda} e^{\left(\sigma_{i}^{2}-2 x \lambda\right) / 2 \lambda^{2}}\left[1-\text { freq } \frac{\sigma_{i}^{2}-x \lambda}{\sigma_{\mathbf{i}} \lambda}\right]
$$

in which the freq function is given in the CERN program library[5.3]

$$
\operatorname{freq}(t)=\frac{1}{\sqrt{2 \pi}} \int_{-\infty}^{t} e^{-u^{2} / 2} d u
$$

The second part of the integration yields,

$$
\left(1-\beta_{i}\right) \int_{-\infty}^{+\infty} S\left(x^{\prime}\right) R_{t a i l s}\left(x-x^{\prime}\right) d x^{\prime}= \begin{cases}\frac{1-\beta_{i}}{\lambda-\mu_{i}}\left[-\frac{1}{2} e^{-x / \mu_{i}}-\frac{\lambda}{\lambda-\mu_{i}} e^{-x / \lambda}\right] & x>0 \\ \frac{1-\beta_{i}}{\lambda+\mu_{i}}\left[-\frac{1}{2} e^{+x / \mu_{i}}\right] & x<0\end{cases}
$$

The full probability density function for a single $\mathrm{B}$ candidate to fall somewhere in the range $-\infty<x<\infty$ is

$$
M_{i}(x)= \begin{cases}\alpha F_{\text {peak }, \mathrm{i}}(x)+(1-\alpha) F_{\text {sidebands }, \mathrm{i}}(x) & \text { for peak region } \\ F_{s i d e b a n d s, i}(x) & \text { for sideband regions }\end{cases}
$$

Many parameters contribute to the form for $M_{i}(x)$, so it is appropriate to give their explanations. Starting with the sideband or background PDF, the $f$ terms are the respective fractions associated with either of the two positive tails or one negative tail. In the original form of the sideband PDF only one high side exponential was used to describe the high side of the distribution. For the relatively small fraction of Run $1 \mathrm{~A}$ data this was adequate, but as more data was added, it became apparent that a second high side tail was necessary. The second high side tail with slope $\lambda_{2}$ accounts for the appearance 
of long lived events in the sidebands. The $30 \mathrm{MeV} / \mathrm{c}^{2}$ gap between the sideband and peak regions is specifically designed to mitigate the chances for real $\mathrm{B}$ mesons entering the sidebands from the decay $\mathbf{B} \rightarrow \Psi \mathrm{K} \pi$. However, as the statistics increase it is quite natural that some longer lived $\mathrm{B}$ mesons trickle into the sidebands.

The peak PDF has a variety of parameters of which some also appear in the sideband PDF. The quantity, $\lambda$, is the mean proper decay length of the distribution while $\sigma_{c r, i}$ is the measurement error of an individual candidate and is an input to the PDF. The term $\beta_{i}$ denotes the fraction of the function $\mathcal{R}$ that is Gaussian. The assumption that $\mathcal{R}$ is completely Gaussian implies that $\beta_{i}=1$. For cases in which $\beta \neq 1$, then $R_{\text {tail }}$ becomes a factor by introducing exponential tails with symmetric slopes, $\mu_{i}$. Although the index $i$ allows for different values of $\beta$ and $\mu$ per candidate, they are all assumed to be the same in the fit to the lifetimes. This is a reasonable assumption since they are parameters of the global shape of the distribution, not of just single events (i.e. it makes little sense to say that a single event is $70 \%$ signal and $30 \%$ background). The quantity, $(1-\alpha)$, gives the background the freedom to adjust to an overall normalization within the peak region. The parameter, $\alpha$, is a measure of the amount of signal in the peak region and typically, this value has been approximately $1 / 4$ for the $B_{u}$ and $B_{d} c \tau$ distributions. An unbinned log-likelihood fit is applied to the $c \tau$ distributions and the free parameters in the default fit are defined in Table 5.1. The likelihood function is the finite product of proper decay length PDF's stated earlier,

$$
L=\frac{e^{-\bar{n}_{\text {peak }}} \bar{n}_{\text {peak }}^{n_{\text {peah }}}}{n_{\text {peak }} !} \cdot \frac{e^{-\bar{n}_{\text {sides }}} \bar{n}_{\text {sides }}^{n_{\text {sides }}}}{n_{\text {sides }} !} \cdot \prod_{i=1}^{n_{\text {pean }}+n_{\text {sides }}} M_{i}(x)
$$

The log-likelihood fit demands that a maximum be found for $\mathrm{L}$, or that $(\mathcal{L})$ given by

$$
\mathcal{L}=-2 \ln (L),
$$

be minimized by MINUIT[5.4]. 


\begin{tabular}{|c|c|}
\hline Fit Parameter & Definition \\
\hline$\lambda=c \tau$ & Mean proper decay length of the signal \\
\hline$\alpha$ & Fraction of signal in the peak region \\
\hline$n_{f i t}$ & Number of fitted signal events \\
\hline$f_{1}^{-}$ & $\begin{array}{l}\text { Fraction of negative tail from resolution effects } \\
\text { in the background }\end{array}$ \\
\hline$f_{1}^{+}$ & $\begin{array}{l}\text { Fraction of positive tail from resolution effects } \\
\text { in the background }\end{array}$ \\
\hline$f_{2}$ & $\begin{array}{l}\text { Fraction of positive tail from long lived } \\
\text { background events }\end{array}$ \\
\hline$\lambda_{1}$ & $\begin{array}{l}\text { Slope of negative and positive tails from } \\
\text { resolution effects in the background }\end{array}$ \\
\hline$\lambda_{2}$ & $\begin{array}{l}\text { Slope of the portion of positive tail } \\
\text { due to long lived background events }\end{array}$ \\
\hline
\end{tabular}

Table 5.1: The eight free parameters in the unbinned log-likelihood fit.

The terms preceding the finite product symbol stem from a couple of observations about the choice of peak and sideband windows. First, since the background shape is assumed to be an extension of the flat sidebands, the number of background candidates is approximately $1 / 2$ the number of sideband candidates for the $B_{d}$ and $B_{u}$. In the case of the $B_{s}$ the number of background candidates is closer to 0.15 the number of sideband candidates since the sidebands are $200 \mathrm{MeV} / \mathrm{c}^{2}$, but the procedure is still the same. In the fit, however, the constraint is not a rigid one, because the background shape is allowed to float within its errors. This is very powerful as it utilizes all the statistics in the sample by taking a fraction of the peak shape of the distribution into account, rather than simply fixing the number background events and then subtracting their $\mathrm{c} \tau$ distribution from that of the peak region. Following this line of logic it is seen that the purity defined as,

$$
\text { purity }=1-\frac{n_{\text {sides }}}{n_{\text {peak }}} \frac{W_{\text {peak }}}{W_{\text {sides }}}
$$


leads to a Lagrange multiplier,

$$
\alpha=1-\frac{\bar{n}_{\text {sides }}}{\bar{n}_{\text {peak }}} \frac{W_{\text {peak }}}{W_{\text {sides }}}
$$

where $n_{\text {peak }}, n_{\text {sides }}, W_{\text {peak }}$, and $W_{\text {sides }}$ are the number of peak events, number of sideband events, width of the peak region and combined width of the two sidebands, respectively. If the fit was a binned fit the peak and sideband regions would be subdivided to calculate the Poisson probabilities per bin. The unbinned fit utilizes the average numbers of events in the peak and sidebands regions as a whole to give an overall probability of an event landing in either of the two regions. The mean numbers of fitted events in the peak and sideband regions are functions of $\alpha$ and the number of fitted signal events,

$$
\begin{gathered}
\bar{n}_{\text {peak }}=\frac{n_{\text {fit }}}{\alpha} \\
\bar{n}_{\text {sides }}=\frac{1-\alpha}{\alpha} \frac{W_{\text {sides }}}{W_{\text {peak }}} n_{\text {fit }}
\end{gathered}
$$

Figures 5.1 through 5.6 show the proper decay length distributions for the $B_{u}, B_{d}$, and $B_{s}$ mesons. Table 5.2 gives the results of parameters returned by the fit. The number of fitted events for the $B_{u}$ is substantially greater than that of the $B_{d}$ due to the additional $K^{*}(892)^{\circ}$ daughter product transverse momentum cuts. The sample purity and fitted values for $\alpha$ are consistent for the three $\mathrm{B}$ sectors. 
RUN $1 A+1 B$ CDF PRELIMINARY
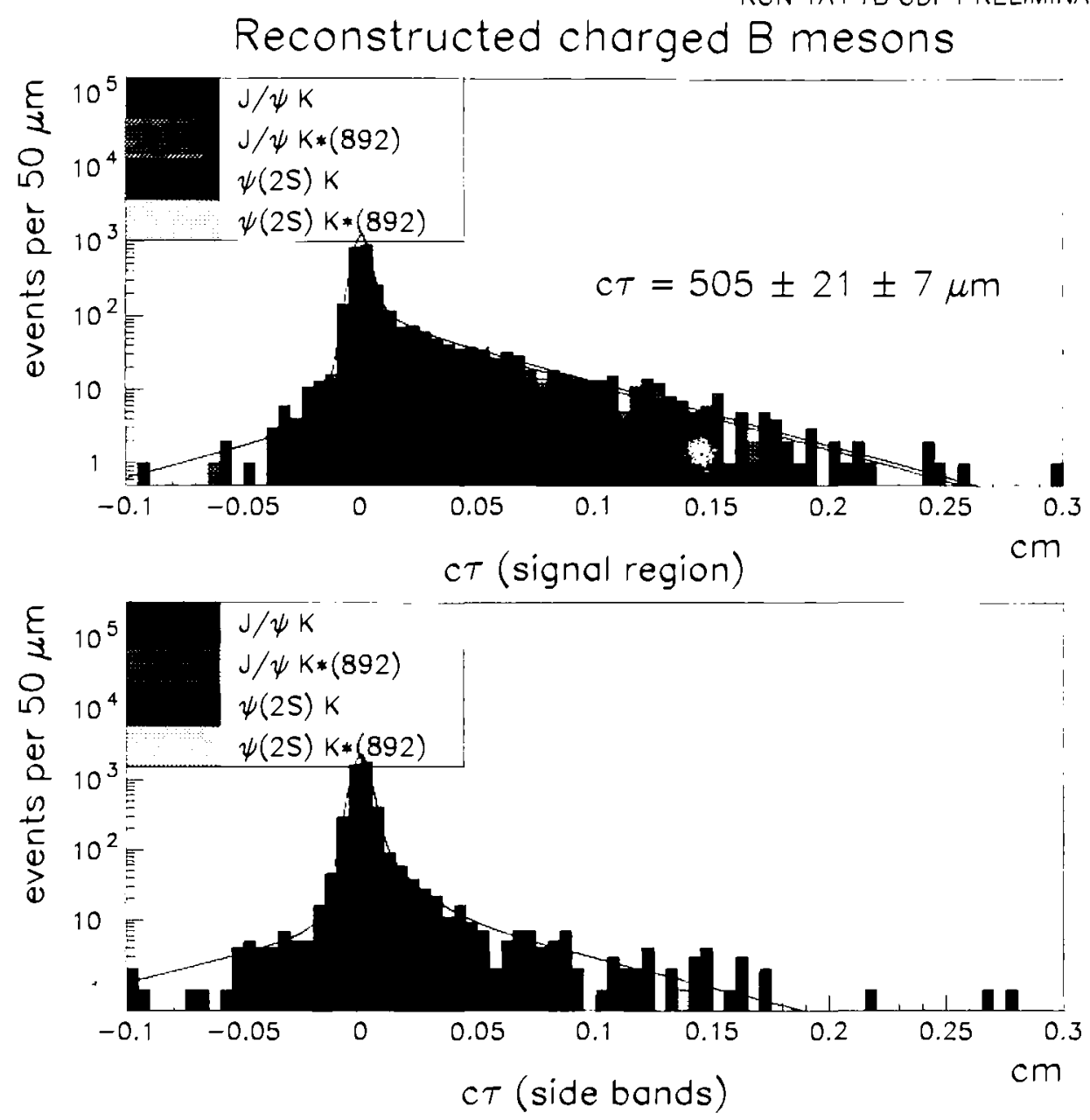

Figure 5.1: Charged B meson proper decay length distributions for the peak(top) and sideband(bottom) regions. The shades of color indicate the relative contributions from each channel. The number quoted is the $c \tau$ with statistical and systematic uncertainties. 

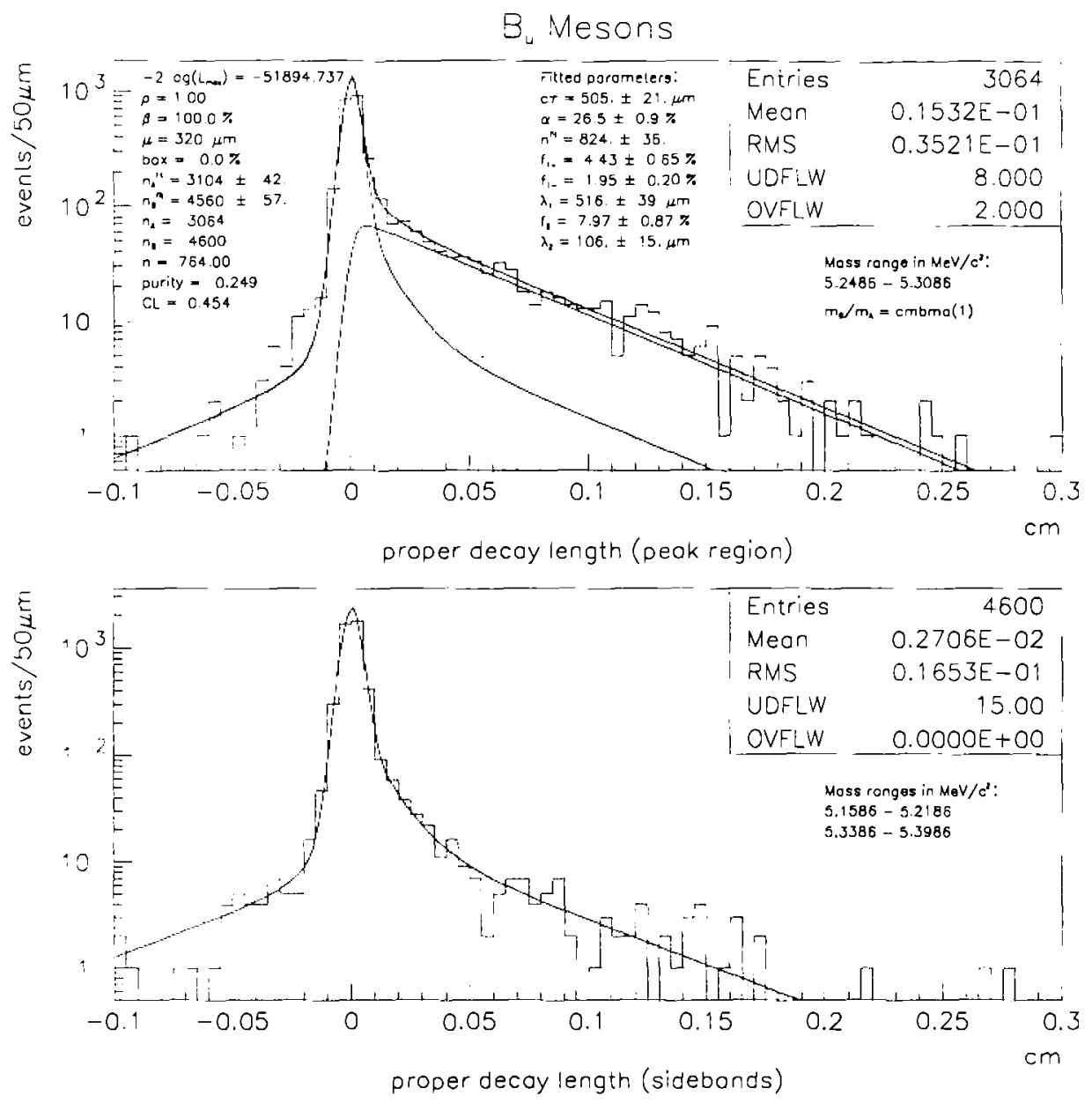

Figure 5.2: Charged B meson $\mathrm{c} \tau$ with the list of fitted parameters. It is the same as Figure 5.1 with a black and white setting to reveal the fitted curves to the peak, background, sideband, and background subtracted distributions. 


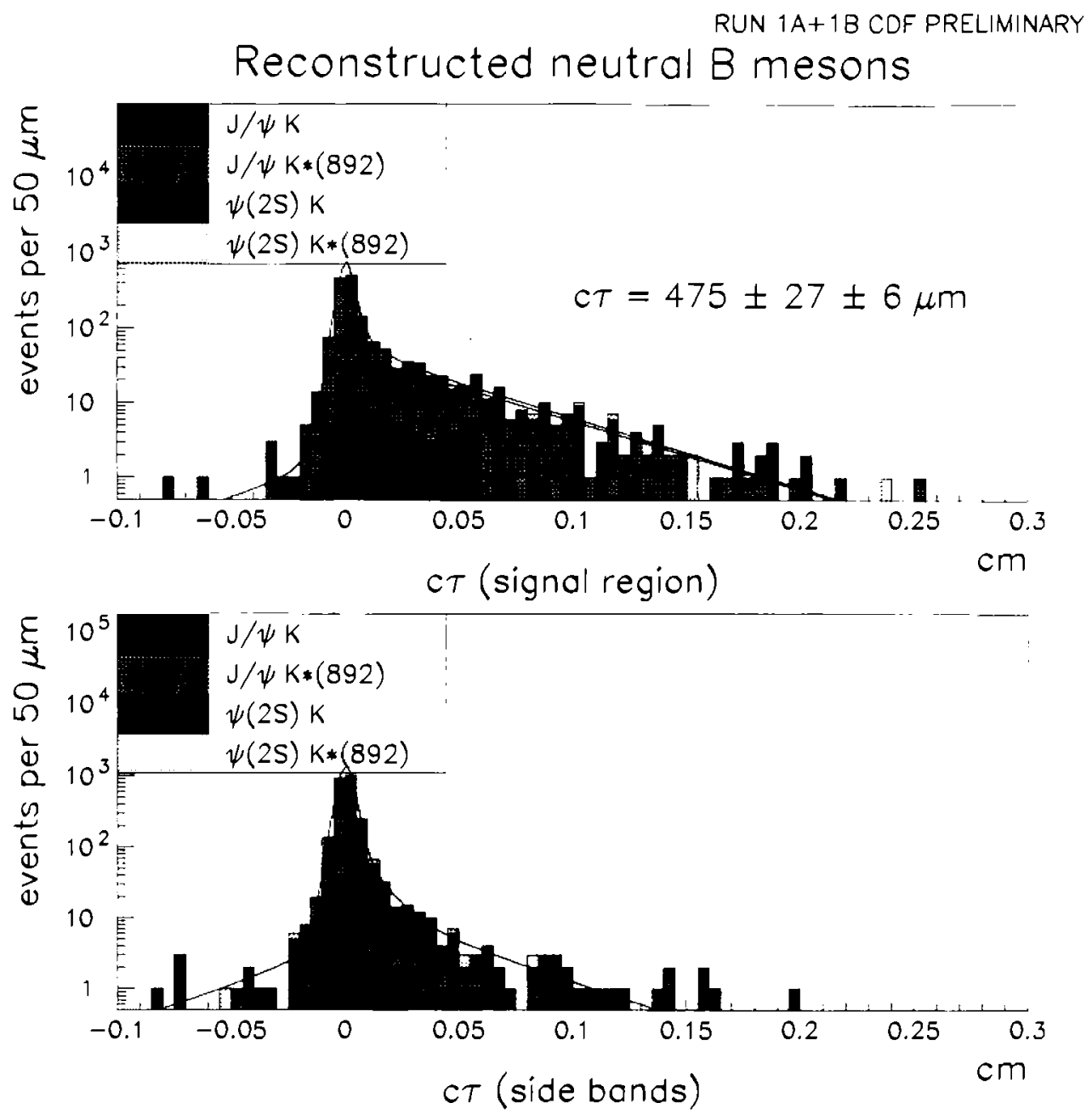

Figure 5.3: Neutral B meson proper decay length distributions for the peak(top) and sideband(bottom) regions. 

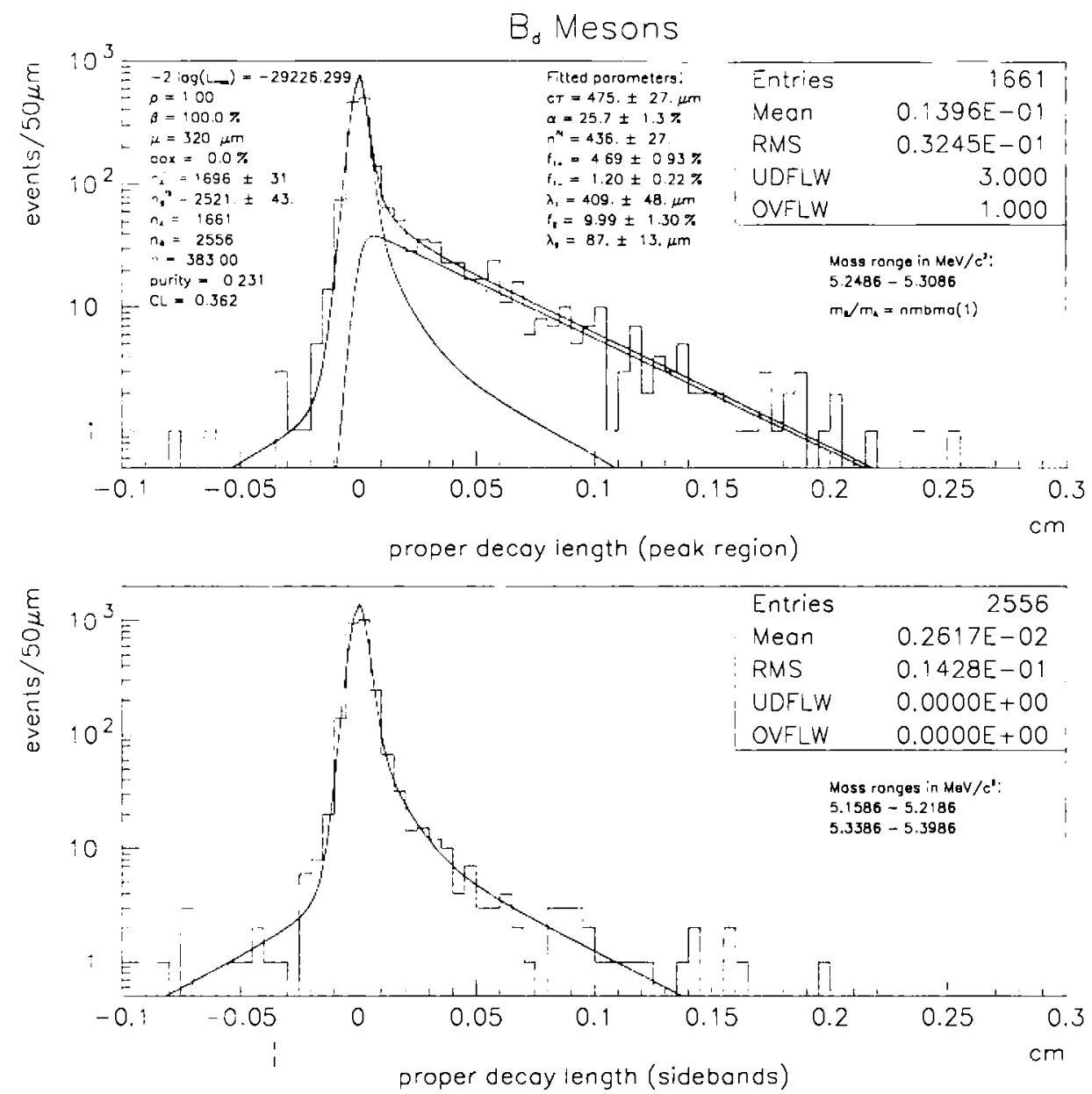

Figure 5.4: Neutral B meson $\mathrm{c} \tau$ with the list of fitted parameters. 
RUN $1 A+1 B$ CDF PRELIMINARY
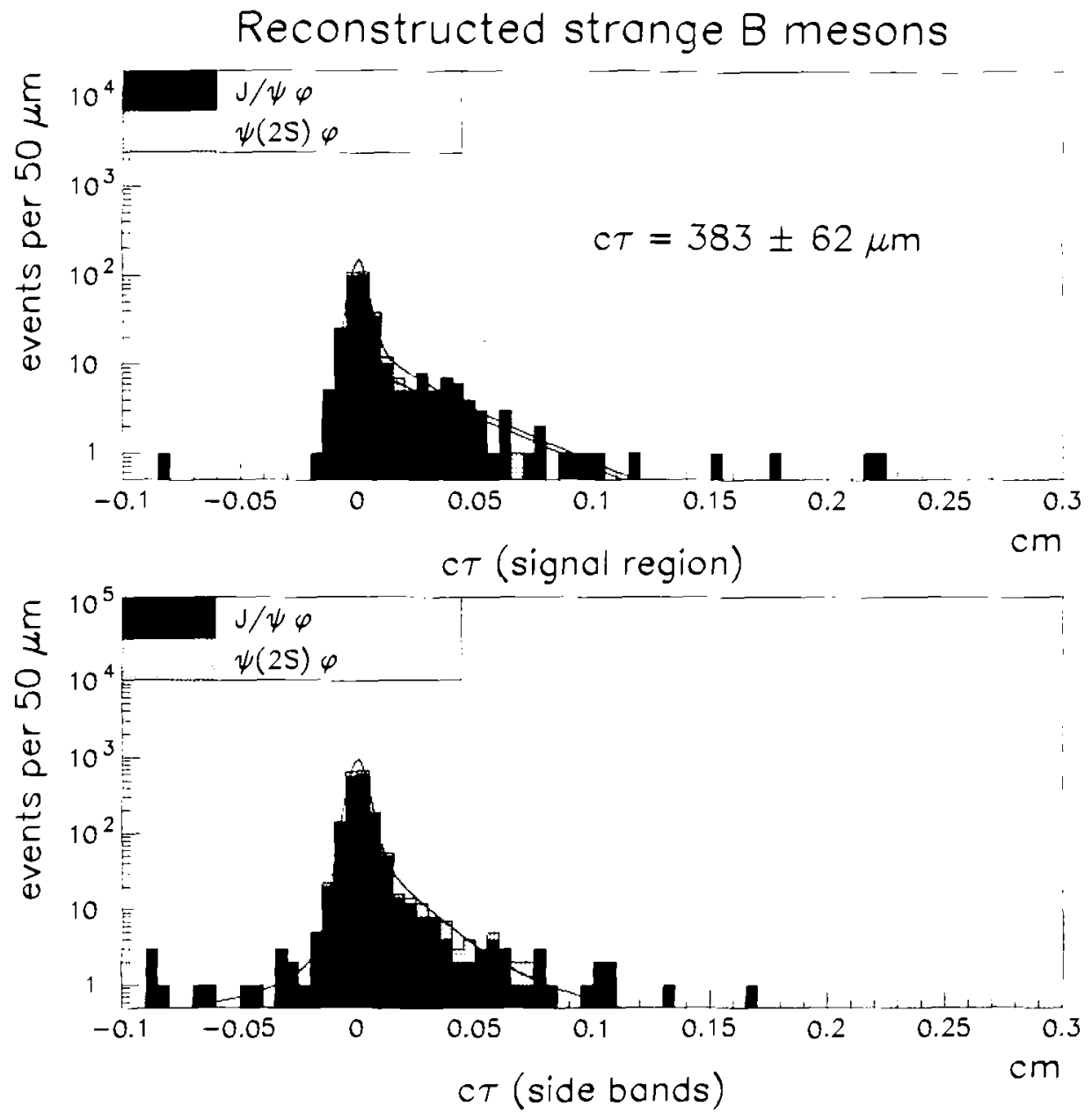

Figure 5.5: Strange B meson proper decay length distributions for the peak(top) and sideband(bottom) regions. The $\mathrm{c} \tau$ is quoted with statistical error only. 

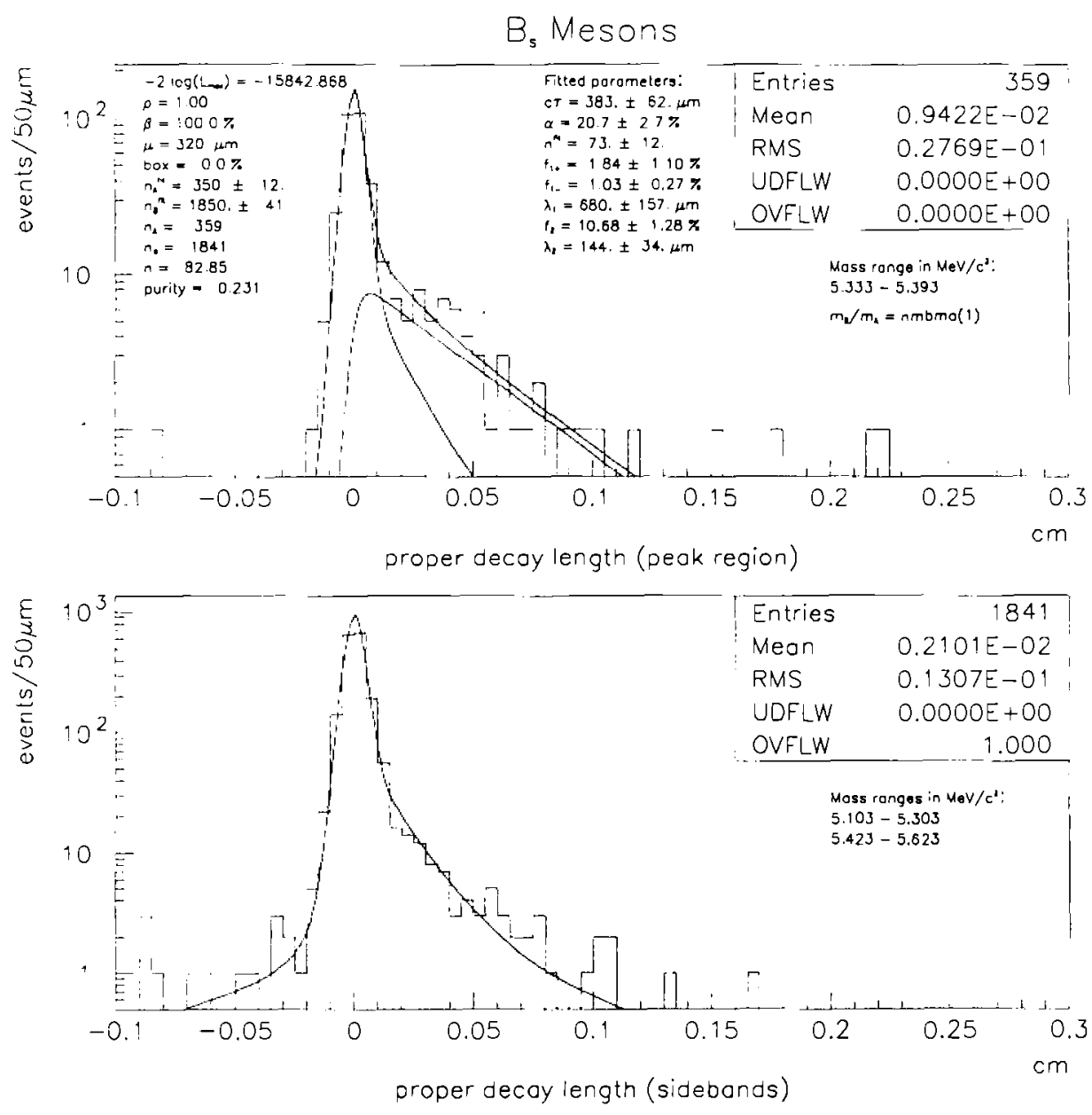

Figure 5.6: Strange B meson $\mathrm{c} \tau$ with the list of fitted parameters. 


\begin{tabular}{|c|l|l|l|}
\hline Parameter & $B_{u}$ & $B_{d}$ & $B_{s}$ \\
\hline$\lambda=c \tau$ & $505 \pm 21 \mu \mathrm{m}$ & $475 \pm 27 \mu \mathrm{m}$ & $383 \pm 62 \mu \mathrm{m}$ \\
$\alpha$ & $26.5 \pm 0.9 \%$ & $25.7 \pm 1.3 \%$ & $20.7 \pm 2.7 \%$ \\
$n_{f i t}$ & $824 \pm 36$ & $436 \pm 27$ & $73 \pm 12$ \\
$f_{1}^{-}$ & $1.95 \pm 0.20 \%$ & $1.20 \pm 0.22 \%$ & $1.03 \pm 0.27 \%$ \\
$f_{1}^{+}$ & $4.43 \pm 0.65 \%$ & $4.69 \pm 0.93 \%$ & $1.84 \pm 1.10 \%$ \\
$f_{2}$ & $7.97 \pm 0.87 \%$ & $9.99 \pm 1.30 \%$ & $10.68 \pm 1.28 \%$ \\
$\lambda_{1}$ & $516 \pm 39 \mu \mathrm{m}$ & $409 \pm 48 \mu \mathrm{m}$ & $680 \pm 157 \mu \mathrm{m}$ \\
$\lambda_{2}$ & $106 \pm 15 \mu \mathrm{m}$ & $87 \pm 13 \mu \mathrm{m}$ & $144 \pm 34 \mu \mathrm{m}$ \\
\hline purity & 0.249 & 0.231 & 0.231 \\
-2ln(L) & -51894 & -29226 & -15843 \\
Confidence level & $45 \%$ & $36 \%$ & $\mathrm{~N} / \mathrm{A}$ \\
\hline \hline
\end{tabular}

Table 5.2: Eight fit parameters with purity and confidence levels.

\subsection{Fit Quality}

Three curves are overlaid in the peak distribution in which one is a fit to the overall peak distribution, a second curve is a fit to the background component derived from the sideband fit, and the third curve is the difference between the first two curves. This last curve contains the slope, $\lambda=c \tau$, of the $\mathrm{B}$ meson distribution. It is seen from the sideband distributions that there are several long lived events in the sidebands which precipitated the second high side exponential term in the fit to the sidebands.

Once the fit is determined, an evaluation of the fit quality is a necessary check. Figures 5.7 through 5.10 show the fit quality for the charged and neutral sectors. For each sector, the fit $\chi^{2}$ per degrees of freedom is determined over several ranges within -.1 to $.3 \mathrm{~cm}$ for the peak, sidebands, and sideband (background) subtracted signal. The number of degrees of freedom is equal to the number of non-empty bins over the specified interval. All the 
distributions have reasonable fit $\chi^{2} / \mathrm{NDF}$ over the entire interval of -.1 to .3 $\mathrm{cm}$.

The confidence levels quoted in Table 5.2 involve Monte Carlo generated proper decay length distributions for the $B_{u}$ and $B_{d}$. First, 1000 samples were generated for each of the two sectors in order to simulate the actual data. For each sample the numbers of peak region and sideband region events are determined by the Poisson distributions contained within the likelihood function:

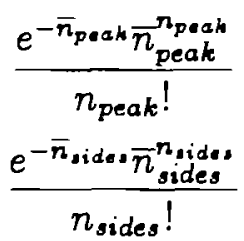

The average numbers of sideband and peak events are those of the actual data. The $\sigma_{c r}$ is found per event governed by the largely Gaussian shape of the errors associated with the peak and sideband regions of the actual data. The proper decay length of the sample is then generated by using the probability density function, $M_{i}(x)$. After applying the simultaneous fit to the sample, the minimized value for $\mathcal{L}$ is obtained and the mean of the c $\tau$ distribution is calculated. The confidence level is the percentage of $\mathcal{L}_{\text {min }}$ values greater than the value obtained from the fit to the actual data. The results of these Monte Carlo distributions are shown in Figures 5.11 and 5.12. A consistency check of the Monte Carlo procedure and error calculation is the observation that the 'Estimate of the error on $c \tau$ ' distribution mean is close to the RMS of the 'Estimate of $\mathrm{c} \tau$ ' distribution indicated in Table 5.3.

\begin{tabular}{|c||l|l|}
\hline \hline$B$ Sector MC & Mean of $\sigma_{c r}$ & $R \mathrm{MS}$ of $\mathrm{c} \tau$ \\
\hline$B_{u}$ & $21 \mu \mathrm{m}$ & $20 \mu \mathrm{m}$ \\
$B_{d}$ & $27 \mu \mathrm{m}$ & $27 \mu \mathrm{m}$ \\
\hline
\end{tabular}

Table 5.3: Values of 'Estimate of the error on $\mathrm{c} \tau$ ' distribution mean compared with the RMS of the 'Estimate of $\mathrm{c} \tau$ ' distribution for the Monte Carlo samples. 
CDF RUN 1A+1B

Sideband subtracted $c \tau$ distributions
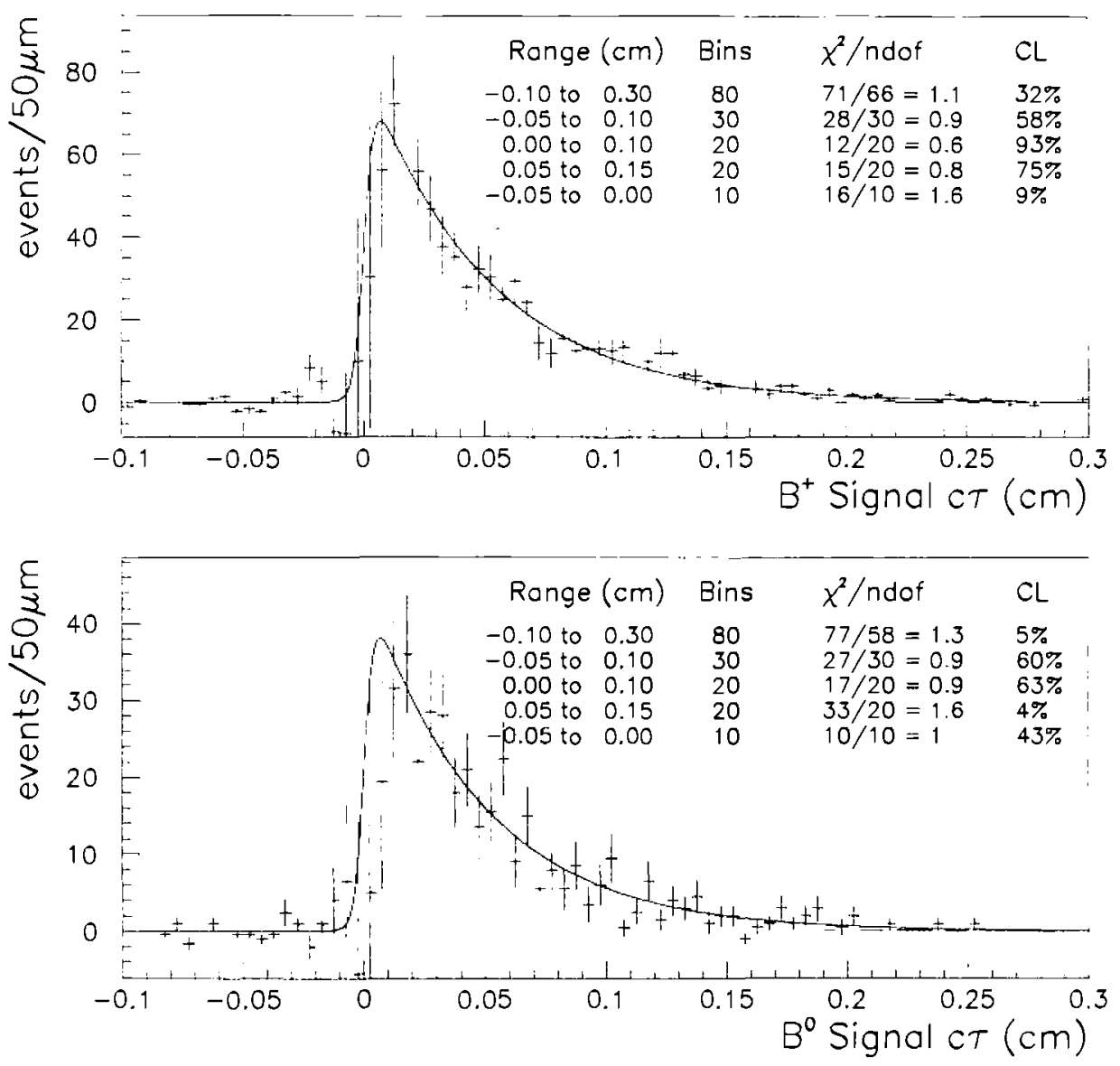

Figure 5.7: Evaluation of fit quality to the peak region. 
CDF RUN $1 A+1 B$
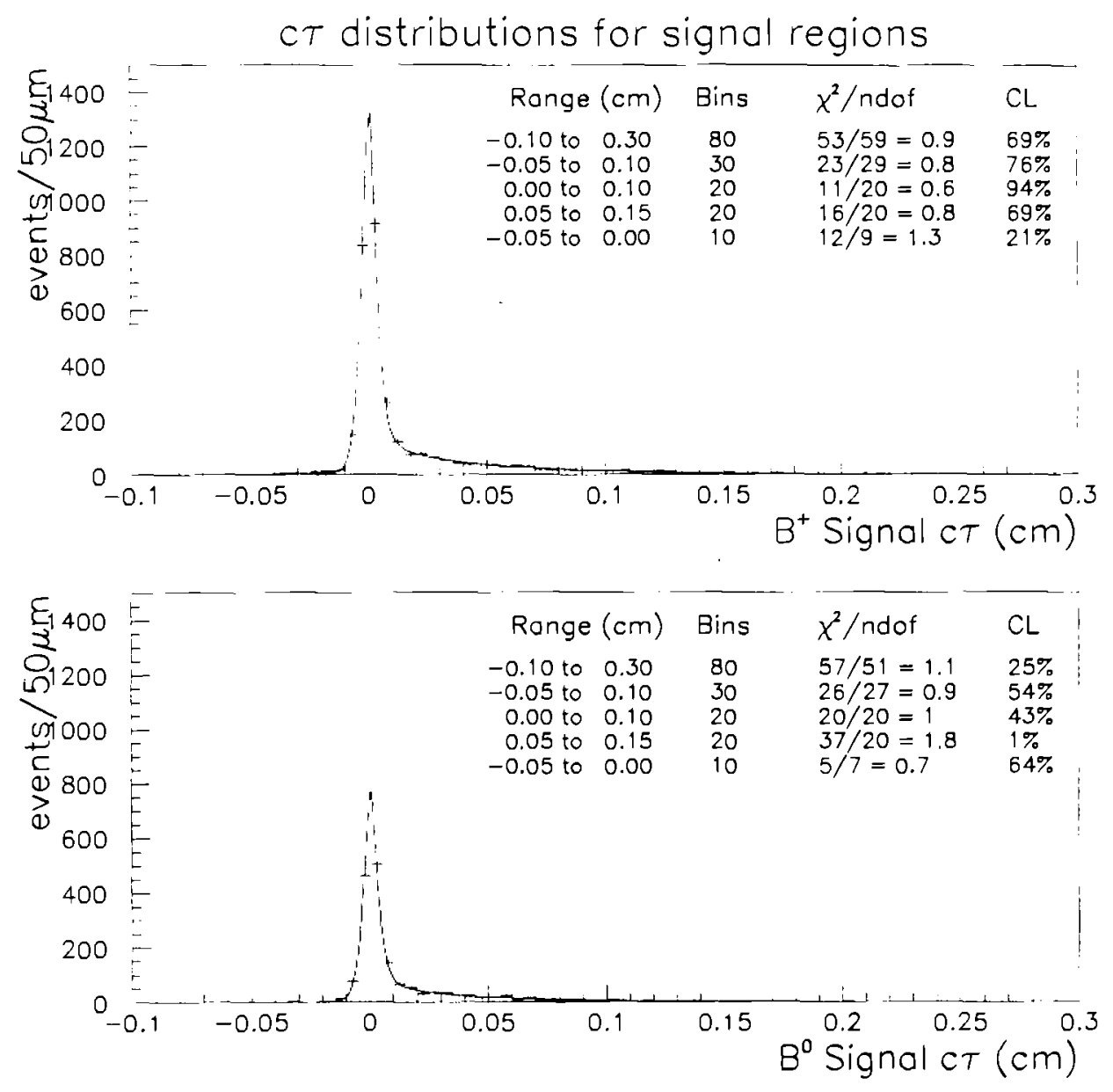

Figure 5.8: Evaluation of fit quality to the sideband subtracted distributions. 


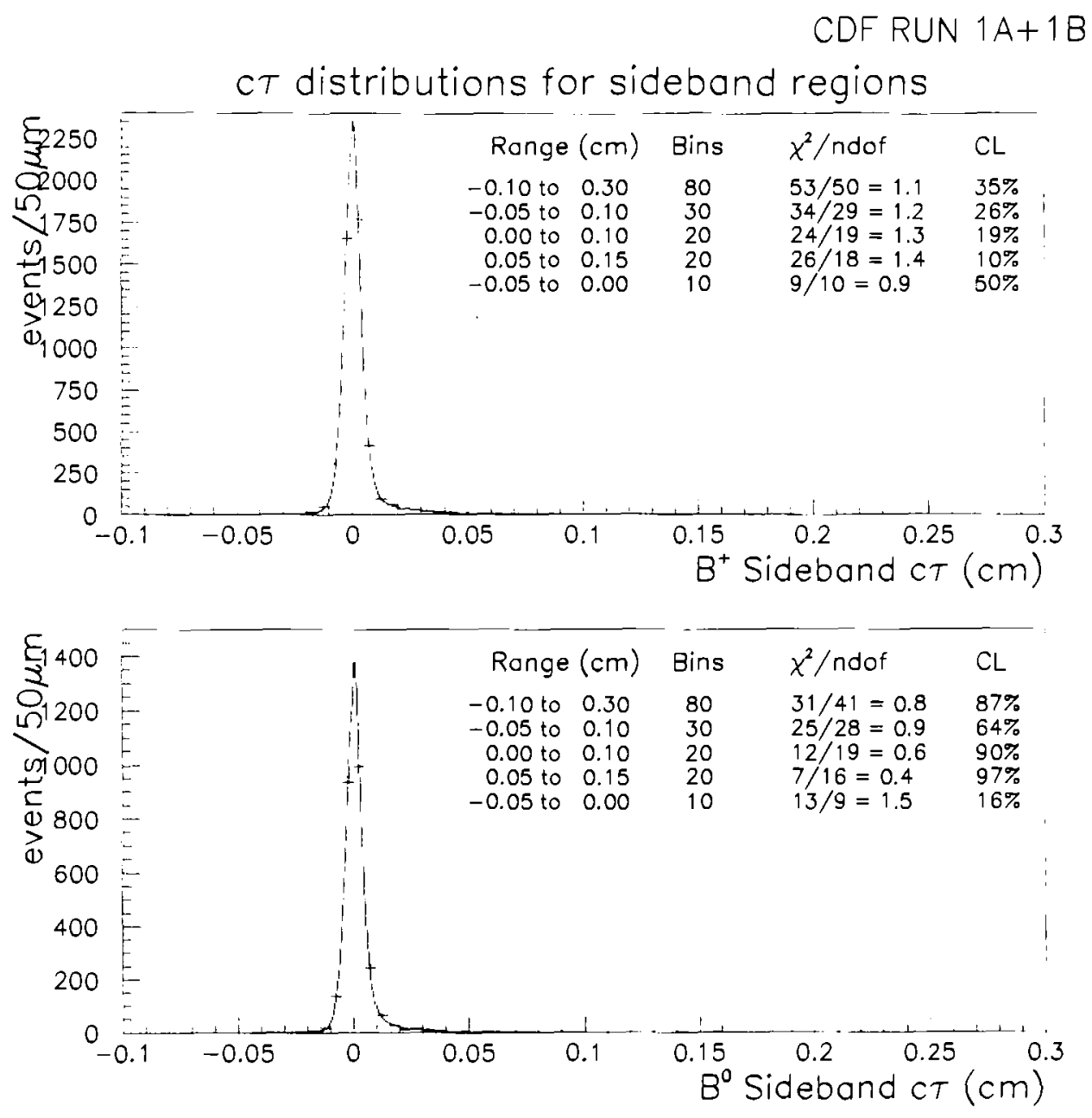

Figure 5.9: Evaluation of fit quality to the sideband distributions. 

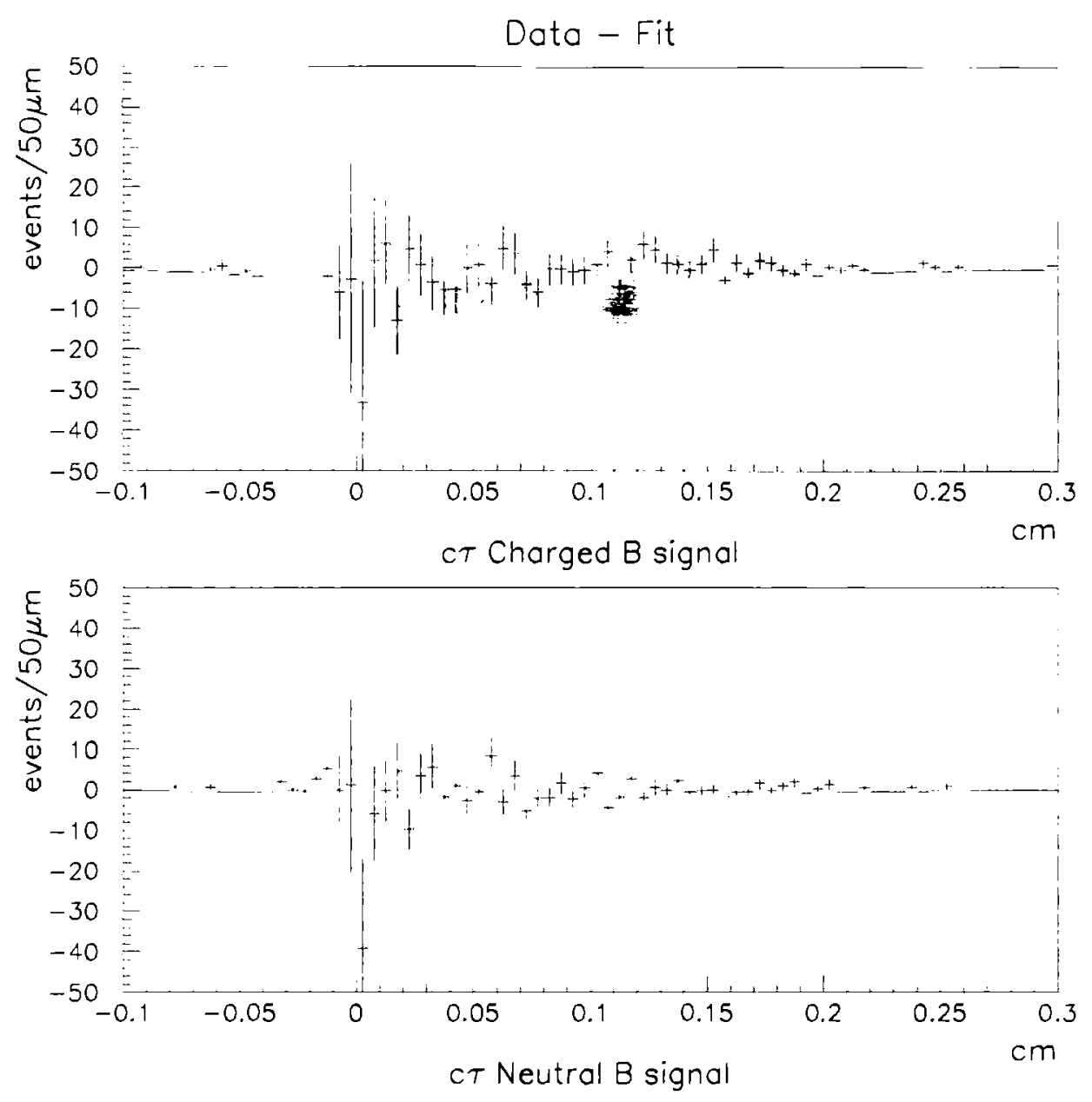

Figure 5.10: A data-fit to the peak distributions. 
The closeness of this pair of values to one another indicates that the error calculation procedure is adequate. To determine statistical error consistency the distribution widths of the plots labeled 'Estimate of the relative error on the lifetime' in the Monte Carlo figures are investigated. The width gives the correlation between the statistical error and the fitted lifetime of the given sample. The relative error width is .0014 and .0024 for the charged and neutral sectors, respectively. This translates into $0.73 \mu \mathrm{m}$ for the charged sector and $1.15 \mu \mathrm{m}$ for the neutral which suggests that the statistical error is calculated to an accuracy within $1 \mu \mathrm{m}$. 


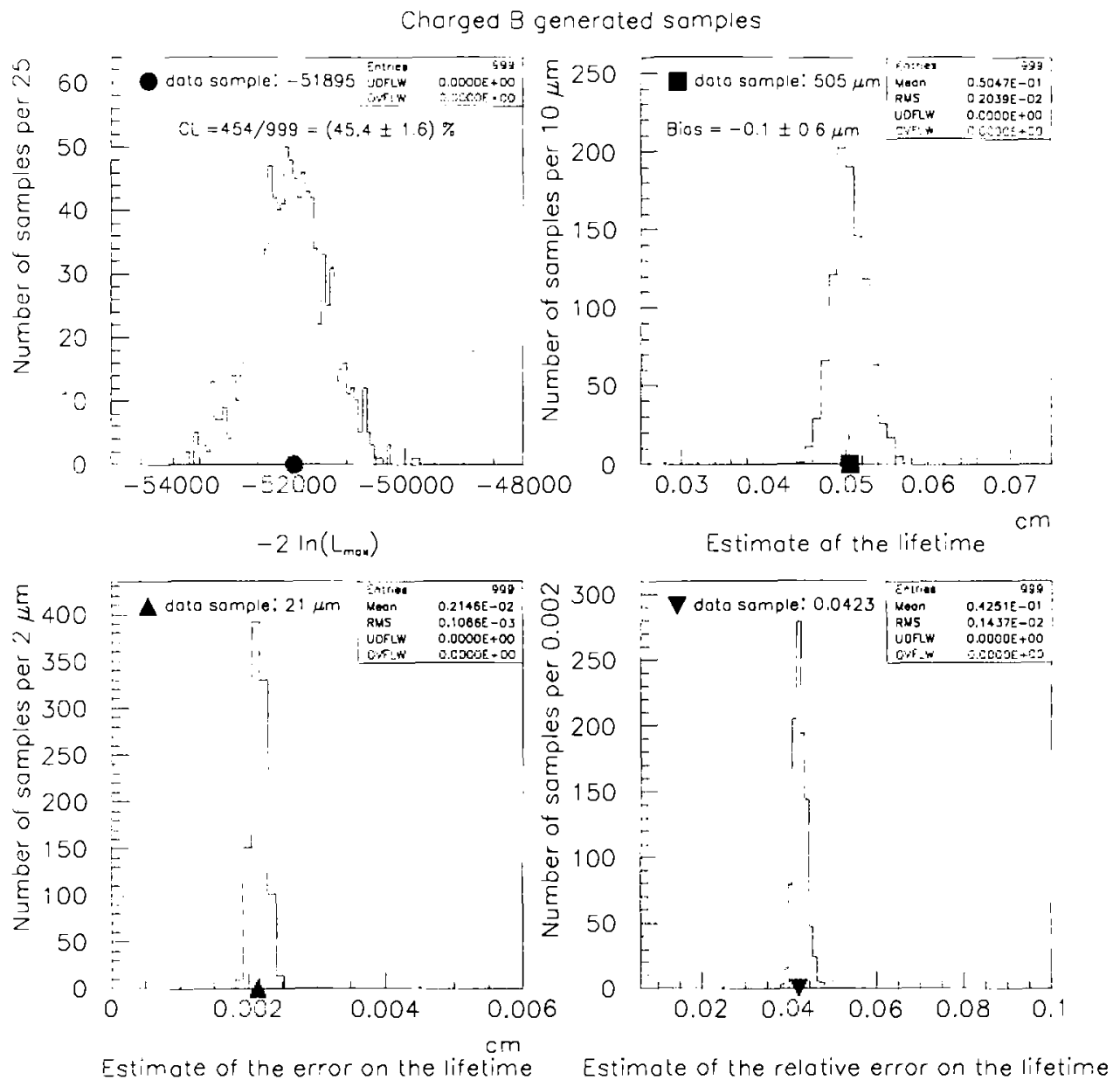

Figure 5.11: Monte Carlo generated $c \tau$ and $\log$-likelihood function values for the charged $\mathrm{B}$ meson sample. The dark points indicate the observed value and the light vertical hashes indicate the sample mean. 

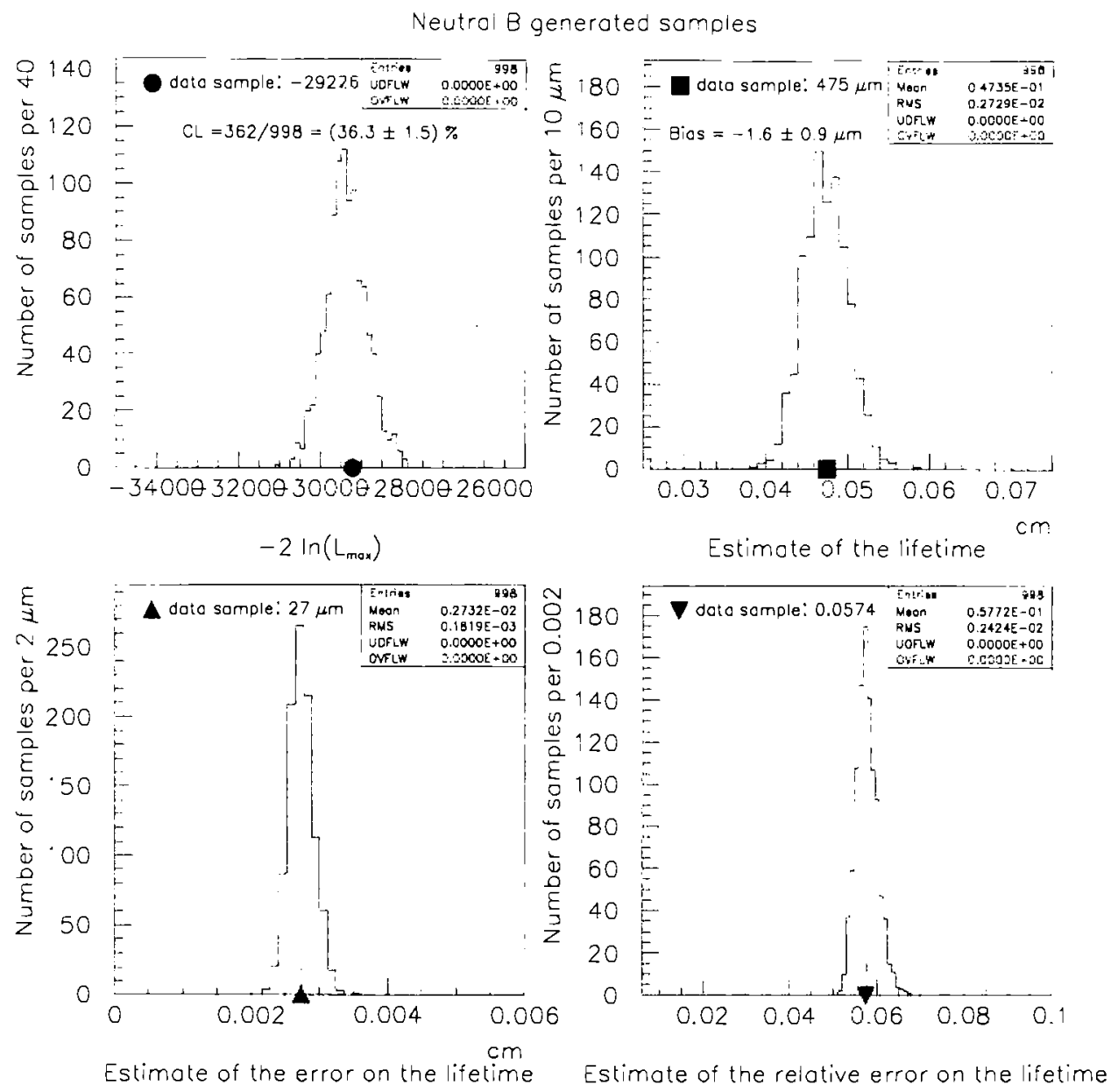

Figure 5.12: Monte Carlo generated $\mathrm{c} \tau$ and log-likelihood function values for the neutral $\mathrm{B}$ meson sample. The dark points indicate the observed value and the light vertical hashes indicate the sample mean. 


\section{Bibliography}

[5.1] Hans Wenzel, et al., Beamlines, CDF internal document number 3334.

[5.2] Hans Wenzel, Fitting the Beam Position with the SVX, CDF internal document number 1924

[5.3] CERN Application Software Group, CERNLIB: Short Writeups CERN (1992).

[5.4] F. Fames and M. Roos, MINUIT: Function Minimization and Error Analysis, CERN (1989). 


\section{Chapter 6}

\section{Systematic Error Analysis}

The dominant error of the exclusive lifetime analysis is statistical and is straightforward to calculate. While the systematic error is small by comparison, the tactics used to calculate its components are a bit more subtle. Systematic error exists within the reprocessed $\mathrm{J} / \psi$ data and the fitting techniques used to extract the lifetimes. The first section outlines the data sample systematics and the second section lists the fit systematics.

\subsection{Data Sample Systematics}

Several analyses involve the reprocessed $7.12 \mathrm{~J} / \psi$ data sample in which the systematics inherent to this sample are the same for all the analyses. Among these are the trigger bias, residual misalignment of the SVX, and the stability of the beam. In principle the trigger can bias the lifetimes since the CFT is less efficient for finding tracks whose origins are relatively far from the beam axis. The CFT efficiency is the dominant component of the trigger bias. For example, each $\mathrm{J} / \psi$ is reconstructed from a dimuon pair. If the $\mathrm{J} / \psi$ emanates from a $B$, the $J / \psi$ vertex is virtually the same as the $B$ vertex. For very long lived $B$ mesons the impact parameters of the corresponding muons from the $\mathrm{J} / \psi$ are large. Since the CFT is less efficient for large 
impact parameter tracks, the possibility exists that these muons may fail Level 2 and thus, longer lived B mesons may be absent from the lifetime distributions. Although all triggers are used in this analysis the dominant number of dimuons comes through the 2-2. Hans Wenzel and Doug Benjamin of the inclusive lifetime group[6.1] measure the CFT' efficiency as a function of impact parameter for the 2-2 trigger and ascertain no discernible loss up to a range of $0.5 \mathrm{~cm}$ which is well beyond the scale on the horizontal axis of the $\mathrm{c} \tau$ plots in Chapter 5 .

Figure 6.1 shows an analogous plot in which the vertical axis represents a ratio of the number of $\mathrm{J} / \psi$ events passing both the 3-1 and 2-2 trigger versus the number which only pass the $3-1$. The horizontal axis is the impact parameter of the lowest $p_{T}$ muon of the dimuon pair. The lower $p_{T}$ muon of each dimuon event is chosen since the one with higher transverse momentum certainly has CFT tracks associated with the 3-1 trigger and, hence, the 2-2 trigger as well. In normalizing to the number of events passing the $3-1$, any bias from the 3-1 trigger is removed leaving only contributions from the 2-2 in the numerator. It is seen from the plot in Figure 6.1 that no loss from the 2-2 is evident which agrees with the inclusive group's conclusion.

Figure 6.2 shows the CFT efficiency as a function of the lower $p_{T}$ muon impact parameter for dimuon events leading to $\mathrm{J} / \psi$ events. The efficiency is taken as the ratio of events that pass TWO_CMU_ONE_CFT_2_2 plus the events that pass the TWO_CMU_TWO_CFT_2_2 trigger versus events that only pass the TWO_CMU_TWO_CFT_2_2 trigger. The plot reveals a similar flat pattern as in Figure 6.1.

The stability of the beam is another concern. If the beam position varies widely over the course of a run then a large systematic error would be associated with extracting the primary vertex from run averaged beam positions. Whether or not the beam drifts during a store[6.2] is irrelevant since the beam positions are determined on a run-by-run basis. The primary concern is how much the beam moves during the course of a run.

Figure 6.3 shows the transverse beam stability in which the errors corre- 


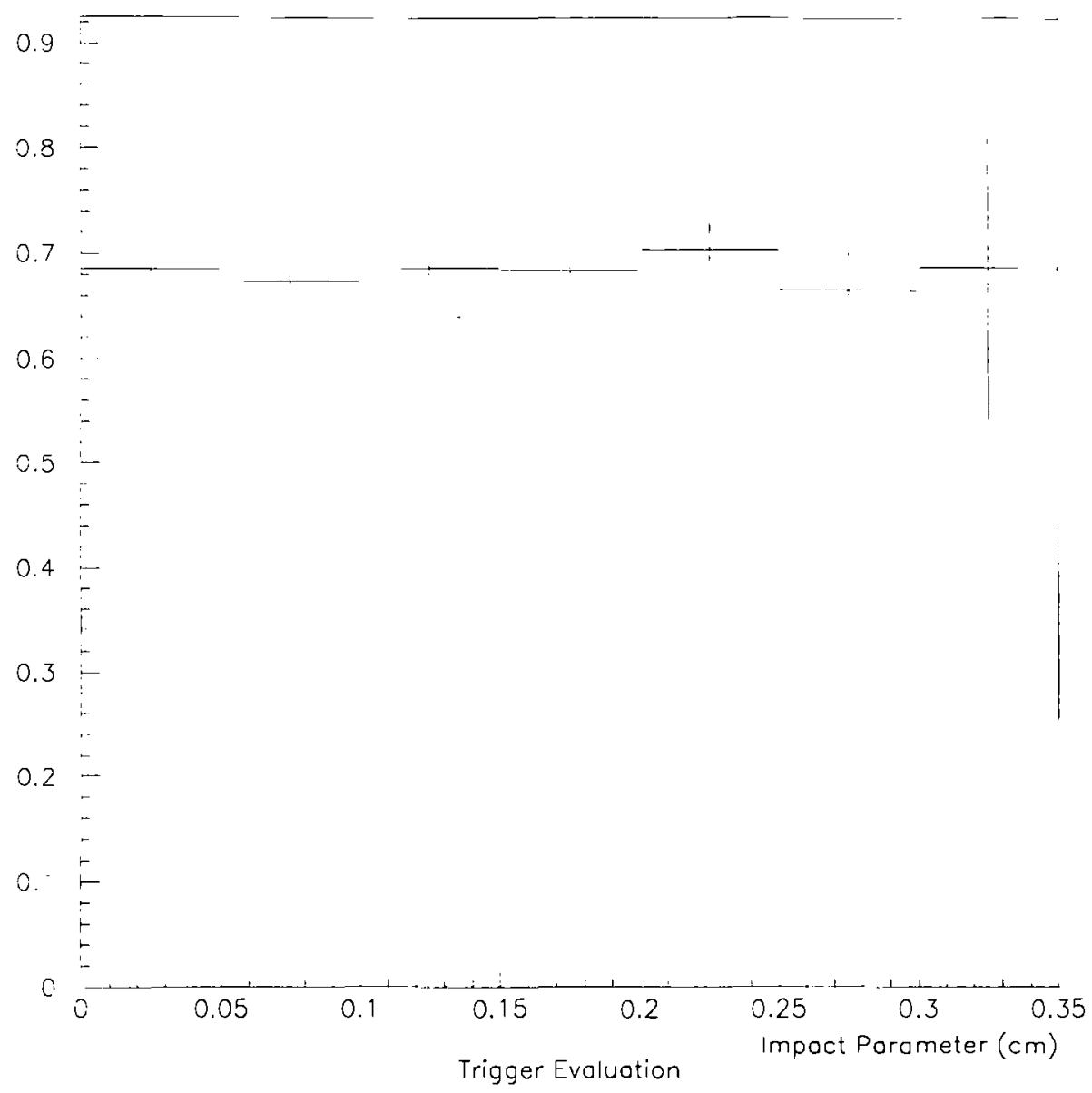

Figure 6.1: An indicator of trigger loss as a function of lowest $p_{T}$ muon impact parameter. The vertical axis represents the ratio of 2-2 $\cap 3-1$ to 3-1 triggered dimuon events leading to $\mathrm{J} / \psi$ 's. 


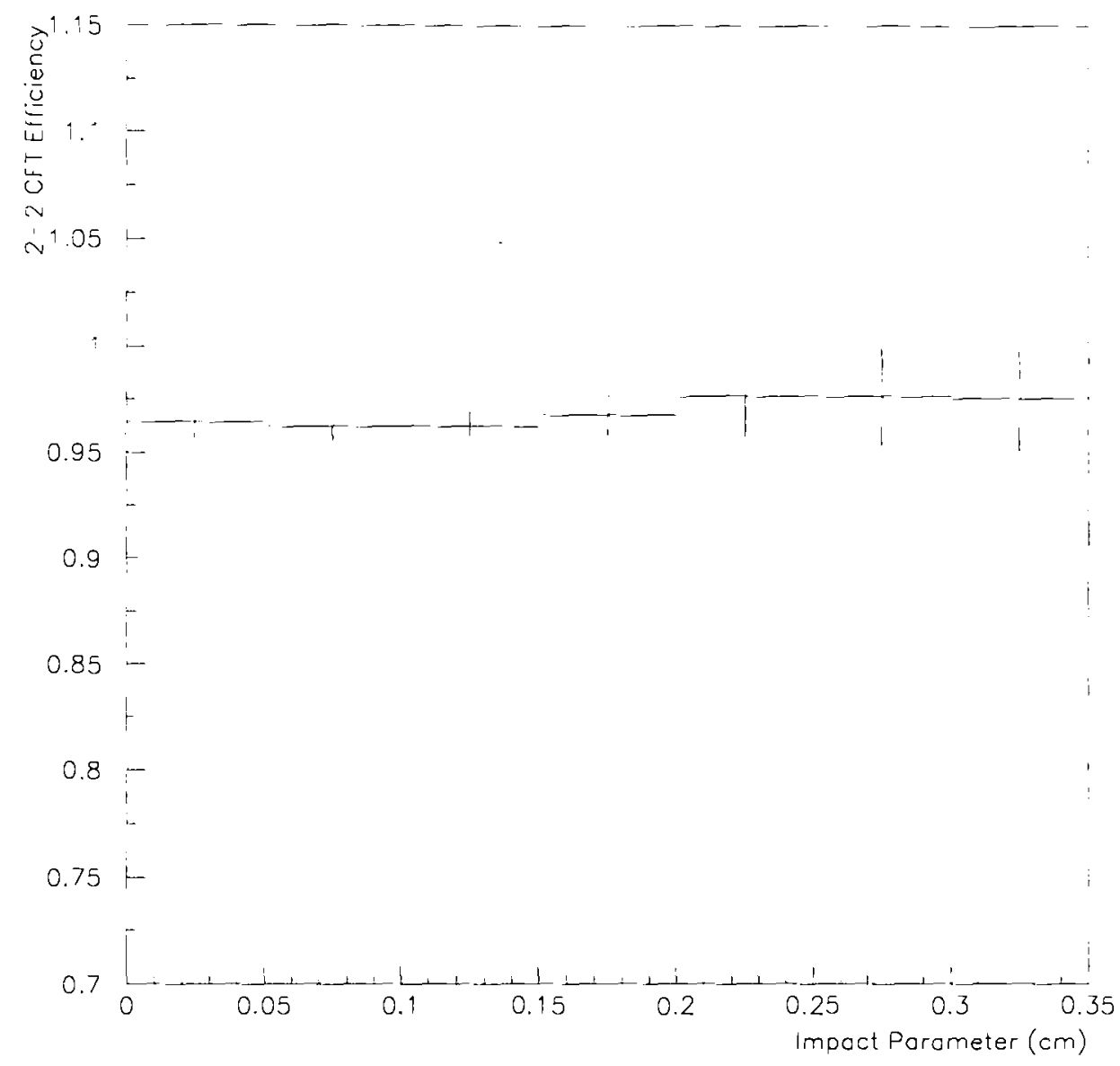

Figure 6.2: The 2-2 CFT efficiency as a function of muon impact parameter. 

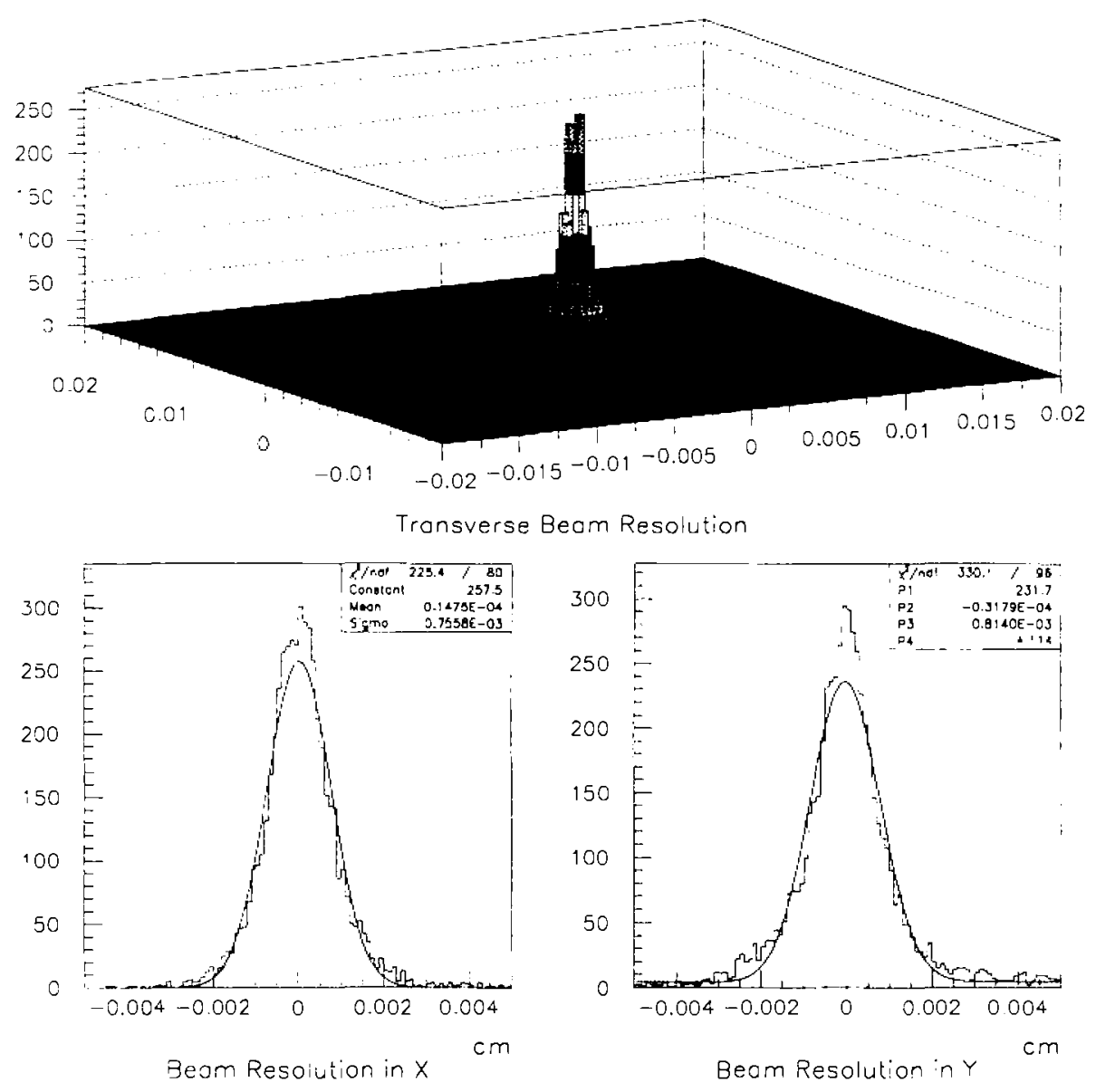

Figure 6.3: Beam stability plots showing the movement of the beam over the course of many runs. The resolutions correspond not to beam spot size but to the actual error on the position of the beam. The transverse errors yield $\sigma_{x}= \pm 7.5 \mu \mathrm{m}$ and $\sigma_{y}= \pm 8.1 \mu \mathrm{m}$. 
spond to the actual deviations in $\mathrm{x}$ and $\mathrm{y}$, not the physical spot size of the beam. For each run the total number of tracks is broken into 1000 track groups and the beam position is calculated using a similar technique as described in Chapter 5 for the extraction of the primary vertex. The horizontal axes of the plots in Figure 6.3 represent the differences between these calculated values and those in the database on a run-by-run basis for most of the runs contained in Run 1B. It is seen that the beam is extremely stable in which $\sigma_{x}= \pm 7.5 \mu \mathrm{m}$ and $\sigma_{y}= \pm 8.1 \mu \mathrm{m}$.

The residual misalignment of the silicon vertex detector is a third major concern within the systematics inherent to the $\mathrm{J} / \psi$ data sample. There are two components to the detector alignment. The first involves its alignment relative to the rest of the CDF detector such as the VTX and CTC. The second component involves the internal alignment of wedges and ladders and it is this component which is of most concern to the lifetimes. Figure 6.4 shows the signed impact parameter sum of several dimuon pairs from $Z^{\circ}$ decays[6.3] as a function of distinct intervals in $\phi$. The $Z^{\circ}$ is produced almost at rest so that the impact parameter sums of the dimuon pairs are all close to $0 \mu \mathrm{m}$. For a perfectly aligned detector the values would be independent of $\phi$. Figure 6.4 shows slight deviations throughout the range in $\phi$ but they are all within $\pm 10 \mu \mathrm{m}$.

For the Run $1 \mathrm{~A}$ analysis the beam stability and the residual misalignment were treated as separate systematics[6.4]. However, Hans Wenzel and Doug Benjamin have demonstrated quite well that the errors on the lifetimes attributed to beam instability and wedge-to-wedge misalignment appear as a combined systematic effect[6.1]. This effect acts to widen the distribution of lifetime errors. By introducing a scale factor on the lifetime errors and refitting the lifetimes with this scale factor, as yet to be determined, double counting of this systematic error is prevented. For example, assume the beam to be offset by some reasonable value. B events are isotropic throughout the range in $\phi$ so that while events traveling in the same direction of the offset give shorter $L_{x y}$, events traveling in the opposite direction of the offset pro- 

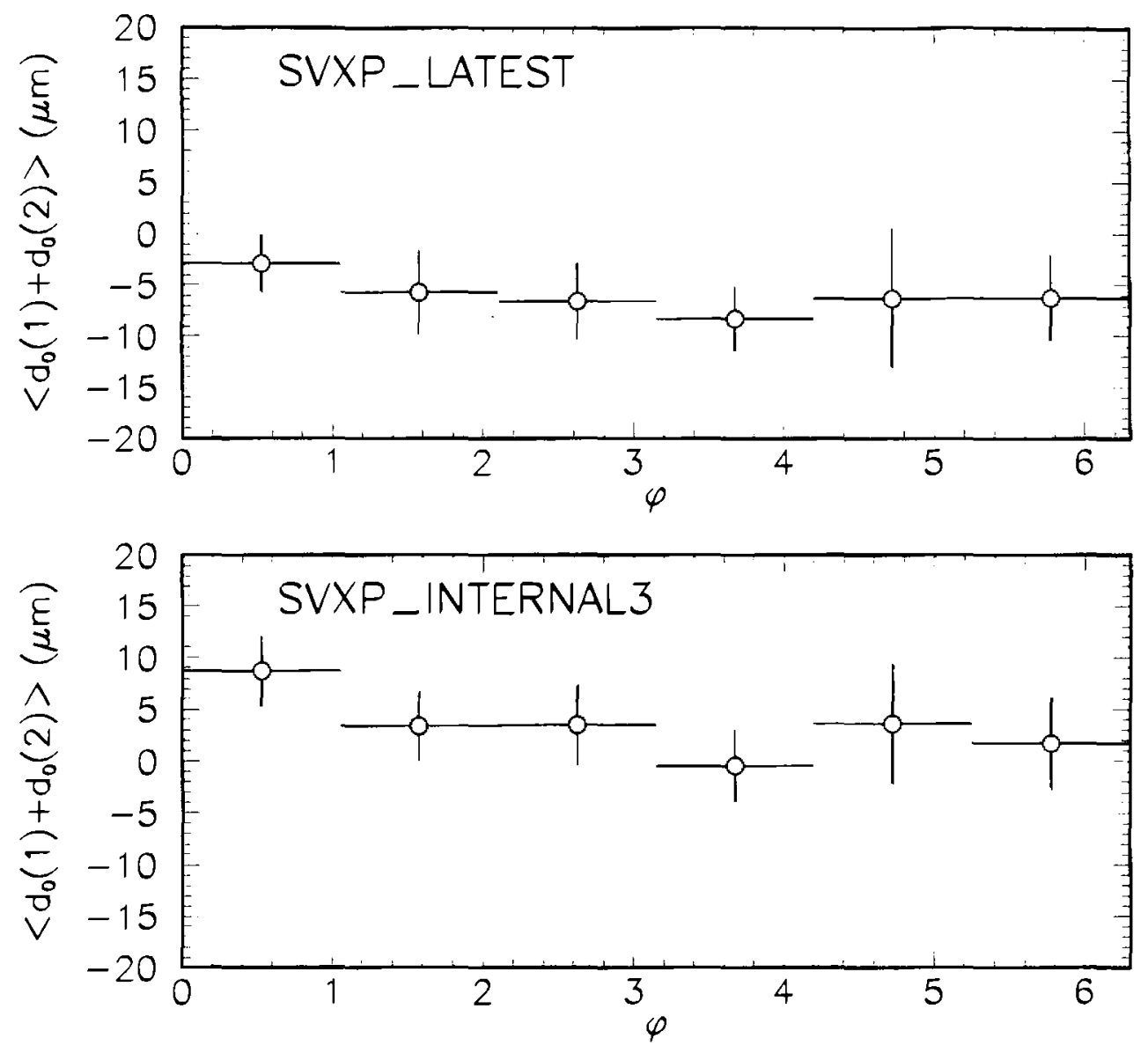

Figure 6.4: Summed impact parameter of dimuon pairs from $Z^{\circ}$ decays over several intervals in $\phi$. The upper plot uses an older SVX alignment data file while the lower plot is for the alignment used in this lifetime analysis. 
duce longer $L_{x y}$. The result is that these shifts on the lifetimes are averaged out over $\phi$, but the lifetime error distribution is wider due to more $B$ events with larger errors in $L_{x y}$. The same scenario exists for the wedge-to-wedge misalignment. This leaves the radial component of SVX misalignment which is labeled as the length scale error. The radial alignment between layers cannot be whisked away into the scale factor of the lifetime errors. The inclusive group [6.1] has calculated the effect on the inclusive lifetime due to radial shifts within the SVX'. These radial shifts are assumed to be up to $80 \mu \mathrm{m}$ based on SVX' alignment studies[6.5] and comparable shifts of less than $100 \mu \mathrm{m}$ are seen for the SVX[6.6]. The $0.3 \%$ systematic error assigned by the inclusive group to this error is taken as the value for the exclusive lifetimes as well since the two groups are using the same $\mathrm{J} / \psi$ data sample and, obviously, the same detector.

\subsection{Fit Systematics}

The second set of systematic errors is specific to the fitting techniques of the exclusive lifetime analysis. Among these is the determination of the scale factor on the lifetime errors. Several options are available for calculating the scale factor that involve prompt particle decays. By fitting the $\mathrm{c} \tau$ significance distribution, the Gaussian resolution width is obtained which is equivalent to a suitable scale factor on the lifetime errors. Figure 6.5 shows the $c \tau$ significance of the large number of prompt $\mathrm{J} / \psi$ states. The width of the distribution is 0.99 (P3 in the fit statistics) implying that the lifetime errors have been overestimated by a factor of $1 \%$. The same distribution is plotted in Figure 6.6 for $\Upsilon(1 \mathrm{~S})$ states. The fit to the distribution is a Gaussian centered at the origin plus two exponential tails, one on each side of the Gaussian. 


\begin{tabular}{|l|c|}
\hline \hline cT Significance Channel & Scale Factor \\
\hline Prompt J/ $/$ states & 0.99 \\
$\Upsilon(1 S)$ states & 0.97 \\
$B_{u} \rightarrow J / \psi K^{+}$Peak Region & 0.96 \\
$B_{u} \rightarrow J / \psi K^{+}$Sidebands & 0.96 \\
$B_{d} \rightarrow J / \psi K^{*}(892)^{o}$ Peak Region & 1.02 \\
$B_{d} \rightarrow J / \psi K^{*}(892)^{o}$ Sidebands & 0.99 \\
\hline
\end{tabular}

Table 6.1: Comparison of the different methods used to determine the scale factor on the lifetime errors.

The resolution scale factor for this distribution is equal to 0.97 (also P3 in the fit statistics). A third method of extracting a value for the resolution scale factor is the use of the highest statistics B meson decay channels. Figure 6.7 shows the $\mathrm{c} \tau / \sigma_{c \tau}$ distributions for the peak and sideband regions of the $B_{u} \rightarrow$ $J / \psi K^{+}$and $B_{d} \rightarrow J / \psi K^{*}(892)^{\circ}$ decay channels. The fits to the distributions are also a Gaussian plus one high side and one low side exponential tail. Table 6.1 lists the results of all the decay channels used to determine the scale factor.

A different approach to finding values for the scale factor involves creating a separate free parameter in the lifetime fit. The free parameter, $\rho$, is the scale factor on the lifetime errors such that, $\sigma_{c \tau, i} \rightarrow \sigma_{c \tau, i} \cdot \rho$. Figures 6.8 and 6.9 show the refitted lifetime distributions in which the values for $\rho$ are 0.98 and 1.01 for the charged and neutral B sectors, respectively. This is in excellent agreement with the values taken from the $\mathrm{c} \tau$ significance fits of the peak region B channels listed in Table 6.1. The differences between the refitted $\mathrm{c} \tau$ distributions and those with the default fit $(\rho=1)$ are virtually transparent. A conservative $1 \mu \mathrm{m}$ error is assigned to both channels due to the uncertainty in $\rho$.

The next systematic specific to the exclusive lifetimes evaluates the assumption that the function $\mathcal{R}$ is completely Gaussian in the probability density function. In looking at the actual resolution functions for the lifetime 


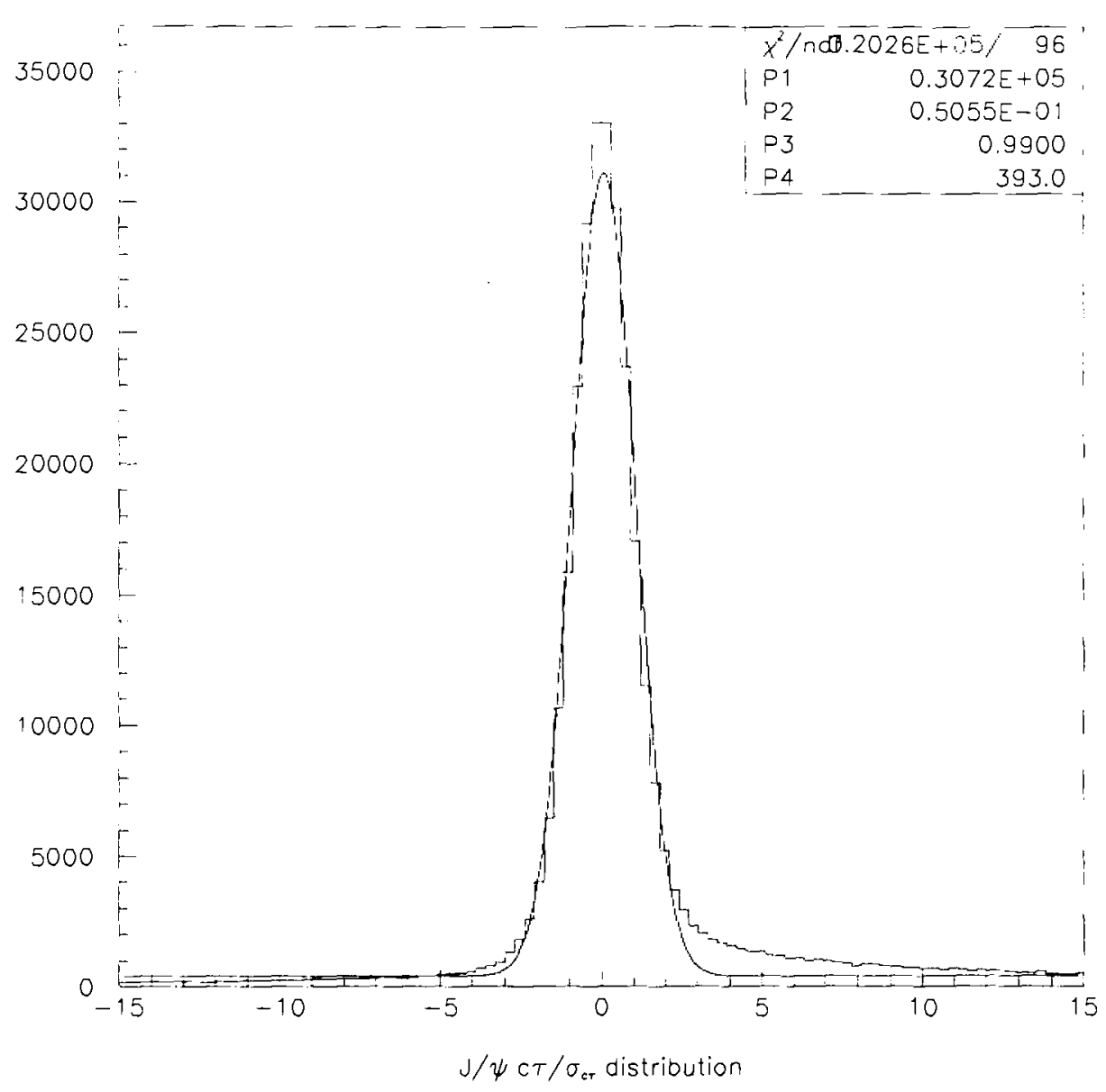

Figure 6.5: $\mathrm{cr} / \sigma_{\mathrm{cr}}$ distribution of $\mathrm{J} / \psi$ states. The ratio gives the width of 0.99 which means that the scale factor on the lifetime errors is close to 1. 


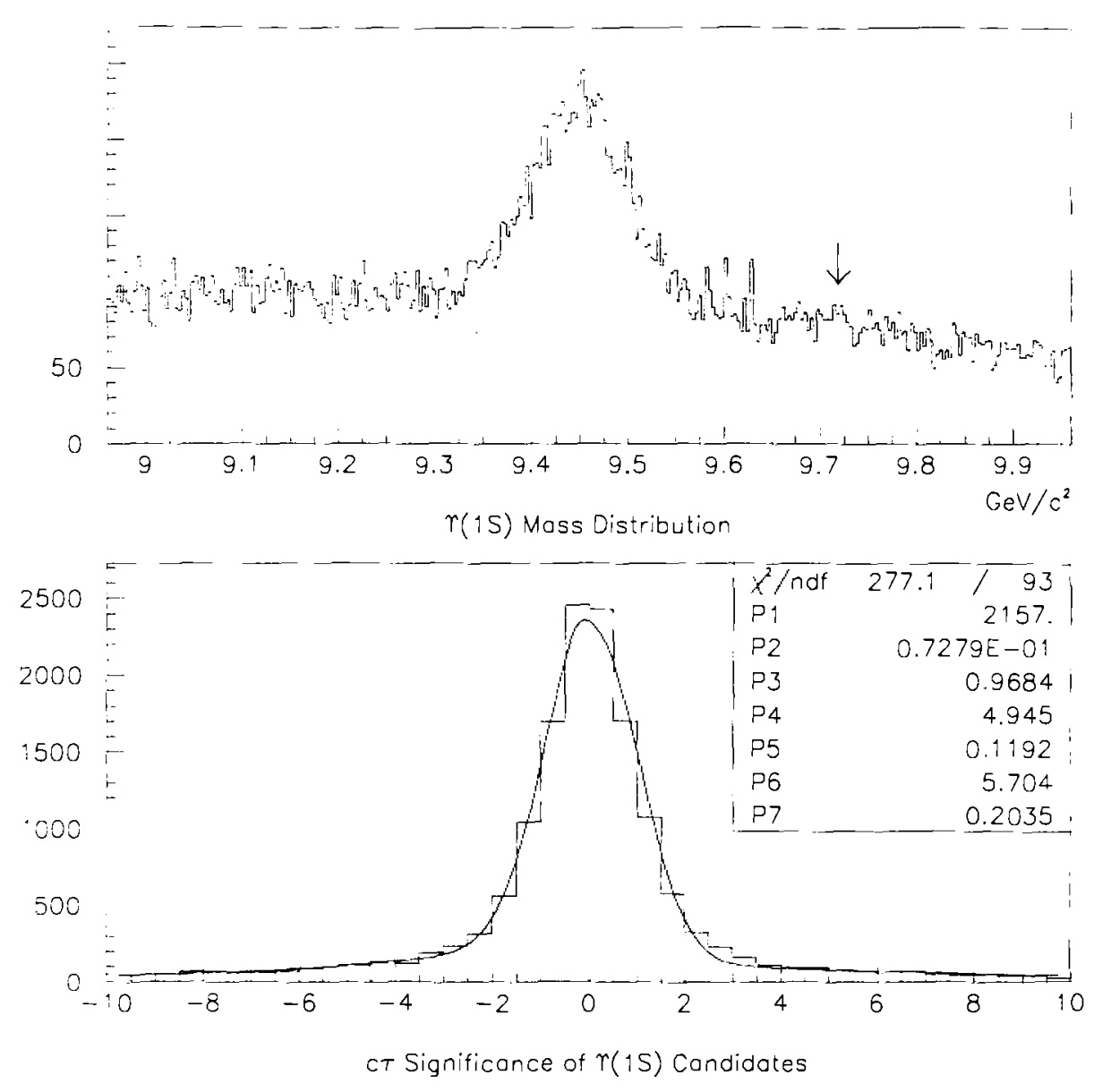

Figure 6.6: $c \tau / \sigma_{c \tau}$ distribution of $\Upsilon(1 \mathrm{~S})$ states. The upper plot shows the $\Upsilon(1 S)$ mass distribution in which the arrows indicate the mass window under consideration for the significance plot beneath it. 



$\mathrm{B}_{u} \rightarrow J / \psi \mathrm{K}^{+}$Sideband Region

$B_{0} \rightarrow J / \psi K^{\cdot}(892)^{\circ}$ Sideband Region

Figure 6.7: $\mathrm{c} \tau / \sigma_{\mathrm{cr}}$ distribution for the high statistics B decay modes. Peak regions and sideband regions are plotted separately for both. 

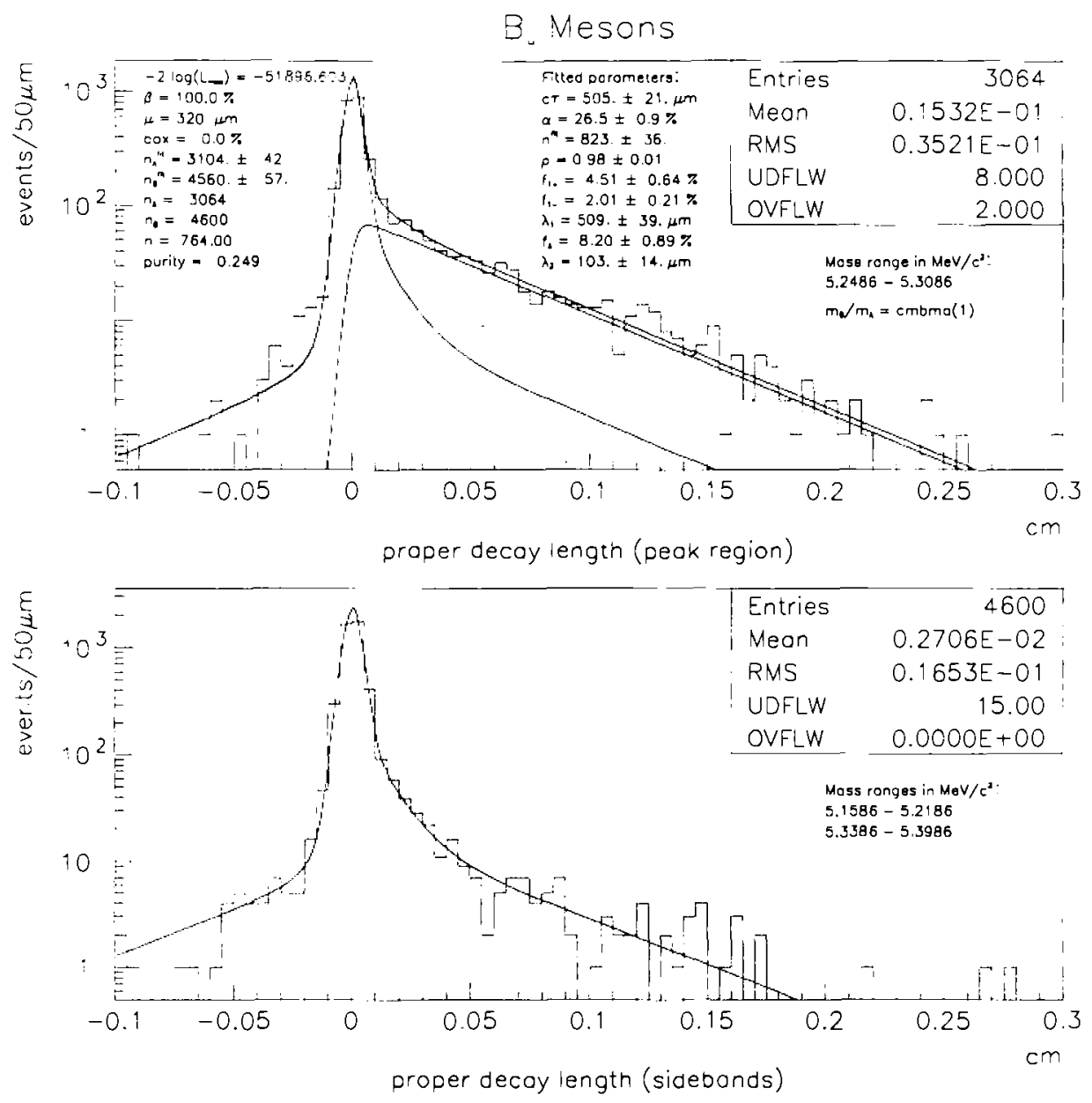

Figure 6.8: Fitted charged lifetime with the additional free parameter, $\rho$, which is the scale factor on the lifetime errors such that $\sigma_{c \tau, i} \rightarrow \sigma_{c \tau, i} \cdot \rho$. 



Figure 6.9: Fitted neutral lifetime with the additional free parameter, $\rho$, which is the scale factor on the lifetime errors such that $\sigma_{c \tau, i} \rightarrow \sigma_{c \tau, i} \cdot \rho$. 
distributions, it is seen that since not all the values for $\sigma_{c \tau, i}$ are the same, the resolution function does not appear Gaussian over the range of $c \tau$ values. Figure 6.10 shows the evidence of this non-Gaussian shape. To an extent this is related to the scale factor error since a wider lifetime error distribution effects the resolution function shape. Because there has been no evidence that they are two different aspects of the same effect, they have been kept as separate systematics.

The hashed curves give the resolution function for the signal and sideband $B_{u}$ and $B_{d}$ sectors. The solid curve represents the Gaussian portion of the resolution function. It is fairly obvious from the plots that the triangular shaped areas on either side of the Gaussian regions are extremely small, accounting for much less than $1 \%$ of the total area. Therefore, the assumption that the function $\mathcal{R}$ is completely Gaussian is very reasonable. Figures 6.11 and 6.12 corroborate this assumption. The lifetimes are refit allowing the $\beta$, the Gaussian fraction of $\mathcal{R}$, and $\mu$, the symmetric exponential slopes of non-Gaussian contributions to $\mathcal{R}$, to be determined simultaneously. The resulting values for $\mathrm{c} \tau$ are $499 \mu \mathrm{m}$ and $470 \mu \mathrm{m}$ for the charge and neutral distributions, respectively. There is little change in the values for $\beta$ in either of the two B sectors. The charged case yields $96.5 \%$ while the neutral sector gives $97.5 \%$. The exponential slopes, $\mu$, are very close to those obtained from fits to the tails in Figure 6.10. The slopes given in Figure 6.10 for the peak region resolution function are $132 \mu \mathrm{m}$ for the $\mathrm{B}_{u}$ and $145 \mu \mathrm{m}$ for the $\mathrm{B}_{d}$ compared with the results of the simultaneous tail fits which yield $124 \mu \mathrm{m}$ $\left(\mathrm{B}_{u}\right)$ and $109 \mu \mathrm{m}\left(\mathrm{B}_{d}\right)$.

A slight change from Run $1 \mathrm{~A}[6.4]$ is the application of $\mathcal{R}$ consistently to the peak and sideband region $B$ mesons. Since the detector is not prejudiced as to whether it is measuring a peak or sideband event, it should measure them with comparable resolution. The systematic errors associated with additional symmetric non-Gaussian tails are $6 \mu \mathrm{m}$ and $5 \mu \mathrm{m}$ for the charged and neutral sectors, respectively. These numbers correspond to the differences in $\mathrm{c} \tau$ returned by the default fits $(\beta=100 \%)$ and the fits described in 
the previous paragraph $(\beta<100 \%)$.

Since the distributions are already modeled with one high and one low side exponential to account for resolution effects, it is no surprise that additional tails are not needed in the fit. The systematic associated with this investigation is a measure as to the extent to which additional symmetric tails are needed. The interchangeability of the modeled tails, $\lambda_{1}$, with those of the resolution function, $\mu$, can be tested by refitting the lifetimes with the fitting fractions $f_{1}^{+}$and $f_{1}^{-}$set to zero. Figures 6.13 and 6.14 do exactly this for the charged and neutral B sectors. The values for $\mathrm{c} \tau$ are only $5 \mu \mathrm{m}$ and 2 $\mu \mathrm{m}$ below the values for the default fit of the charged and neutral $\mathrm{B}$ sectors, respectively. Figures 6.15 and 6.16 check the quality of this alternate fit for the sideband subtracted and peak region $\mathrm{c} \tau$ distributions. In all cases the fit $\chi^{2} / \mathrm{NDF}$ are comparable to those of the default fit.

A final check of the modeling of the symmetric exponential tails is to let the high side and low side resolution tails have different exponential slopes. Actually, this is the first modification to the fitting technique from Run 1A. The results shown in Figures 6.17 and 6.18 show that the values for the slopes, indicated by $\lambda_{-}$and $\lambda_{+}$are almost equal. It is this observation that leads to their restriction that they be made equal. There is no change in the value for $\mathrm{c} \tau$ in the charged case and only a $3 \mu \mathrm{m}$ difference in the neutral sector.

\begin{tabular}{|c|l|l|}
\hline \hline Systematic $(\mu \mathrm{m})$ & $B_{u}$ & $B_{d}$ \\
\hline Scale factor & 1 & 1 \\
Non-Gaussian tails & 6 & 5 \\
Fitting procedure bias & 1 & 2 \\
Length Scale & 2 & 2 \\
\hline Total Systematic Error & 7 & 6 \\
\hline
\end{tabular}

Table 6.2: Summary of systematic errors. The length scale error is completely correlated across the two B sectors. 

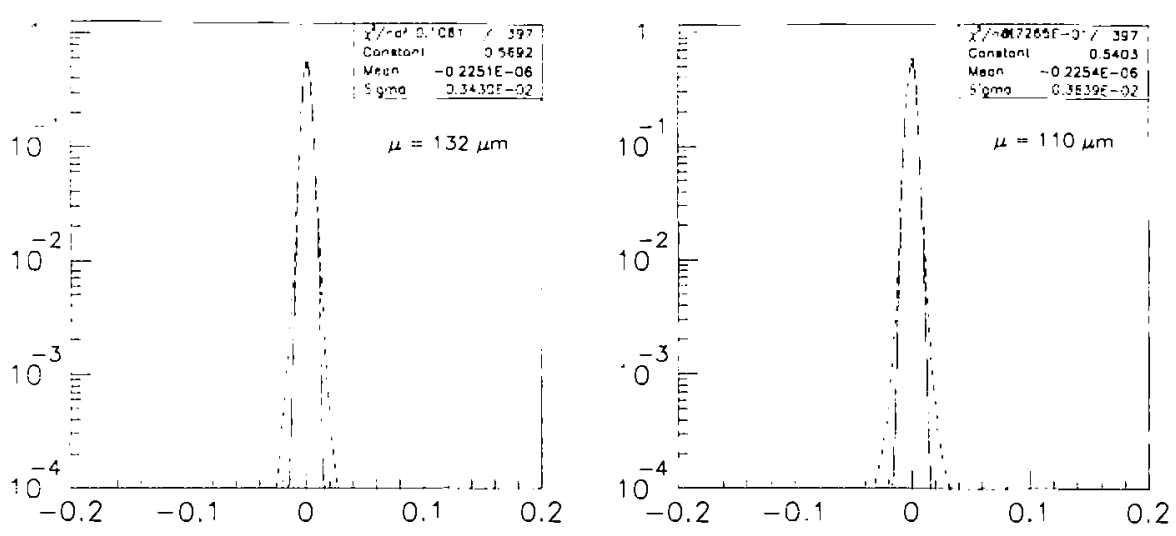

$B_{u}$ Peok Resolution Function

$\mathrm{B}_{\mathrm{u}}$ Sideband Resolution Function
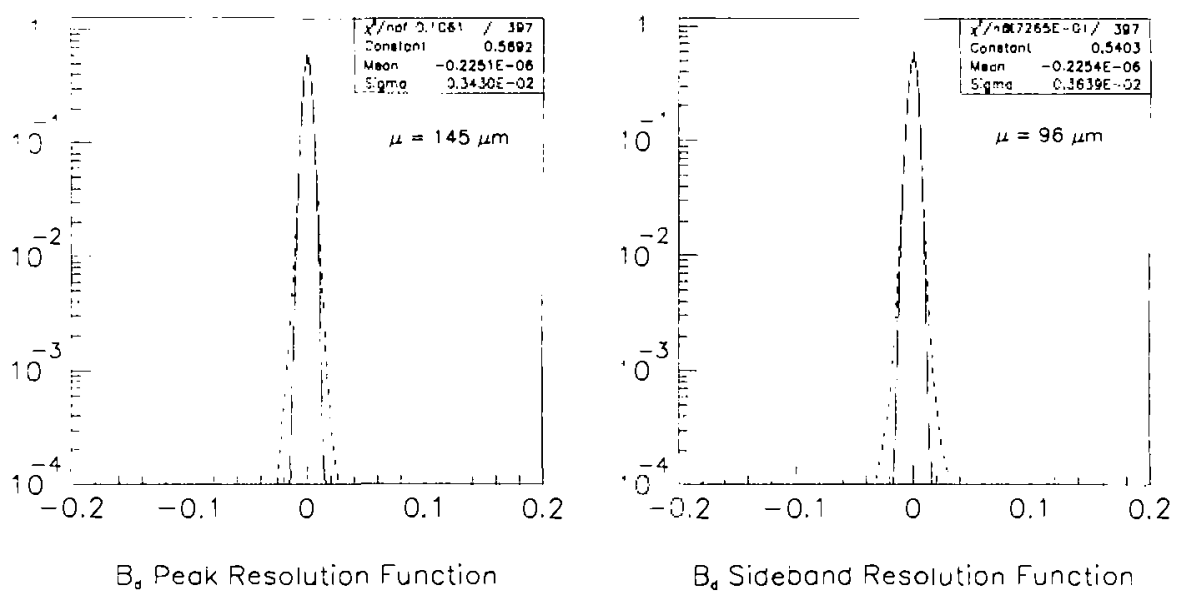

Figure 6.10: The resolution functions for peak and sideband regions. The hashed curve indicates the actual resolution function while the solid curve is the Gaussian portion. 



Figure 6.11: Charged B Meson $c \tau$ distribution for the case of non-Gaussian tails in the resolution function, $\mathcal{R}$. $\beta$ is the percentage of the resolution function which is Gaussian and $\mu$ is the magnitude of the symmetric exponential slopes of the non-Gaussian tails. 



Figure 6.12: Neutral B Meson $c \tau$ distribution for the case of non-Gaussian tails in $\mathcal{R}$. 

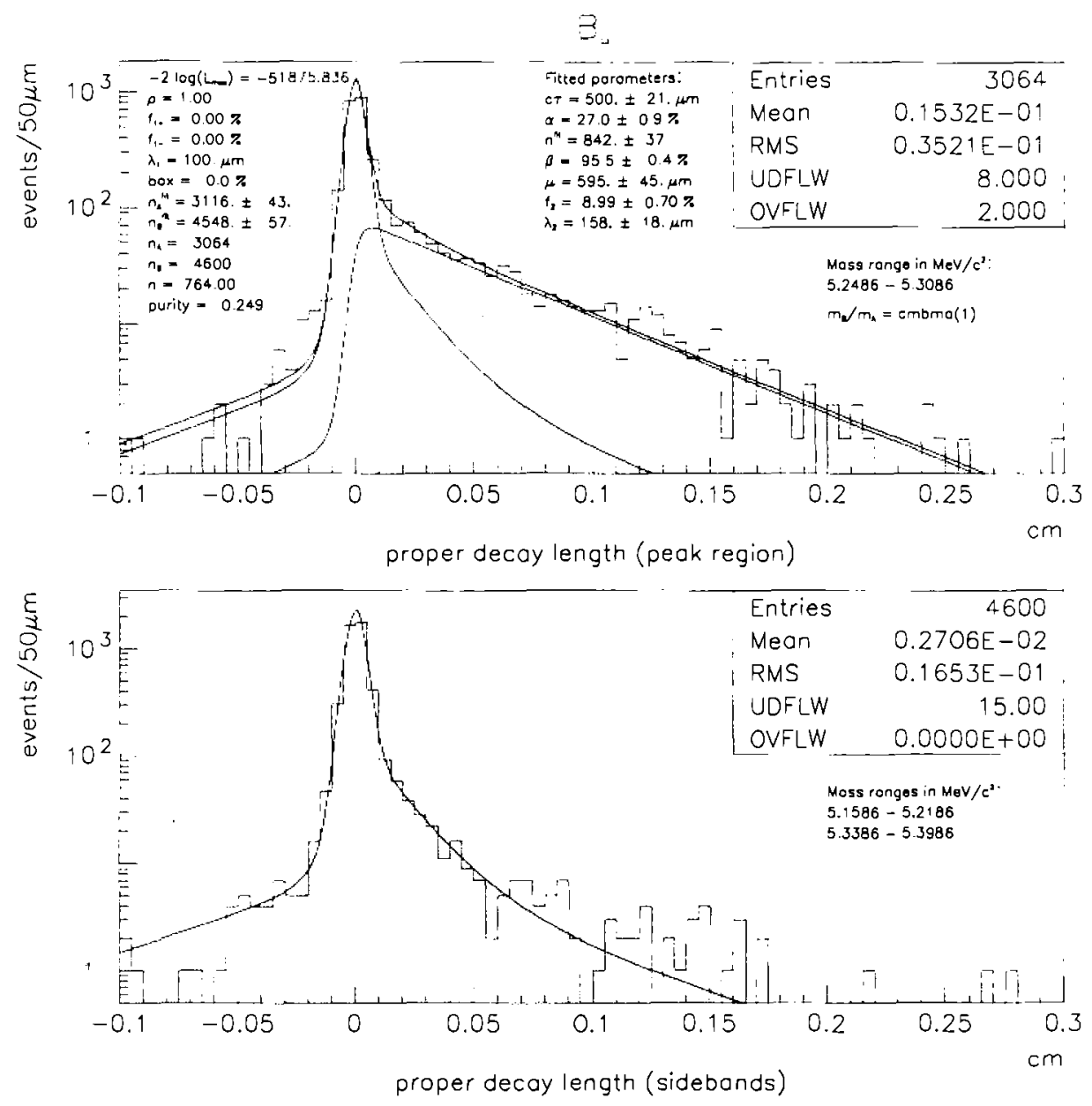

Figure 6.13: Fitted charged $c \tau$ distributions in which the high and low side resolution tails are modeled by non-Gaussian contributions from $\mathcal{R}$. 



Figure 6.14: Fitted neutral $c \tau$ distributions in which the high and low side resolution tails are modeled by non-Gaussian contributions from $\mathcal{R}$. 

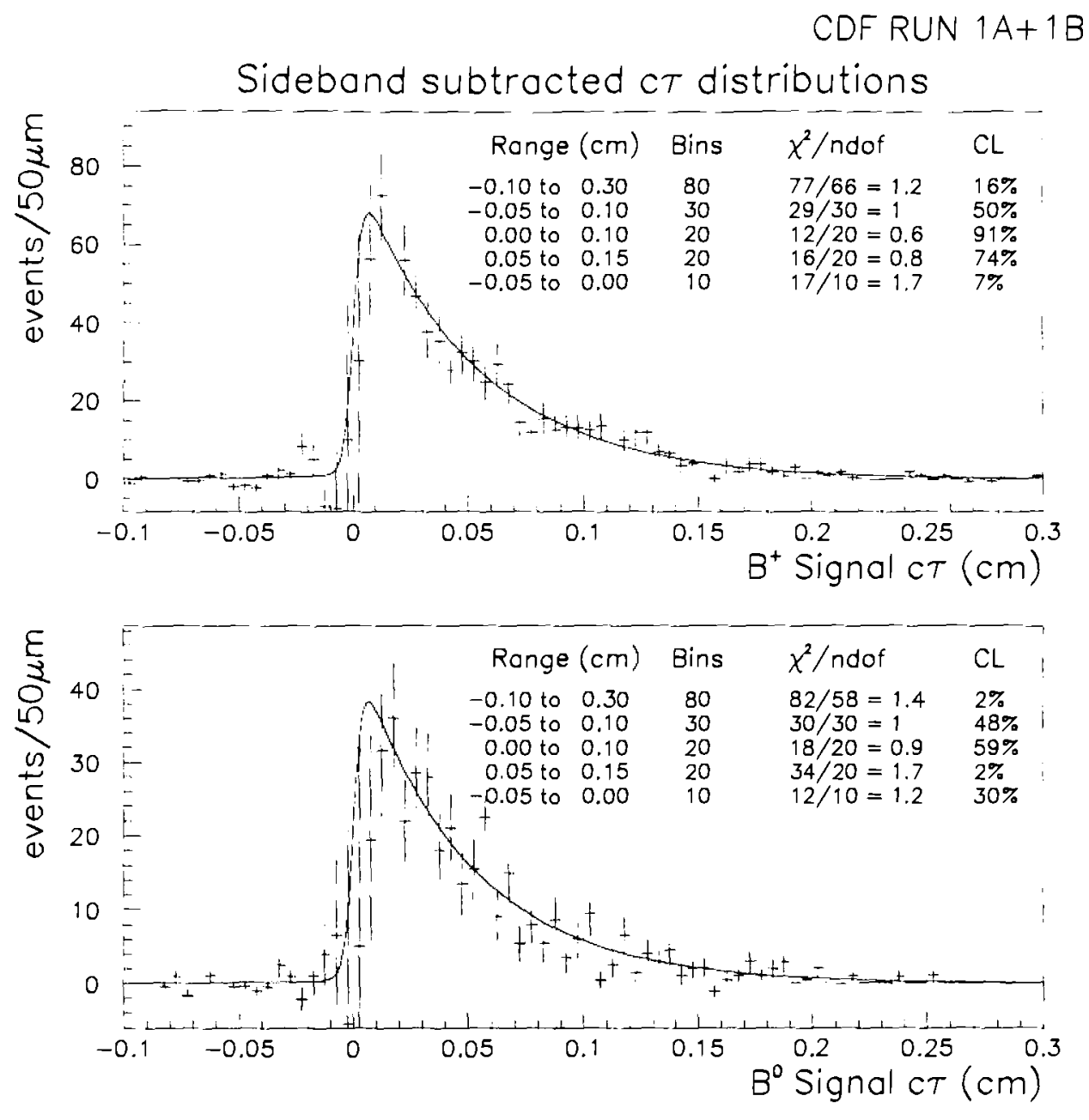

Figure 6.15: Evaluation of the fit to the sideband subtracted distributions for the modeling of non-Gaussian contributions by $\mathcal{R}$. 


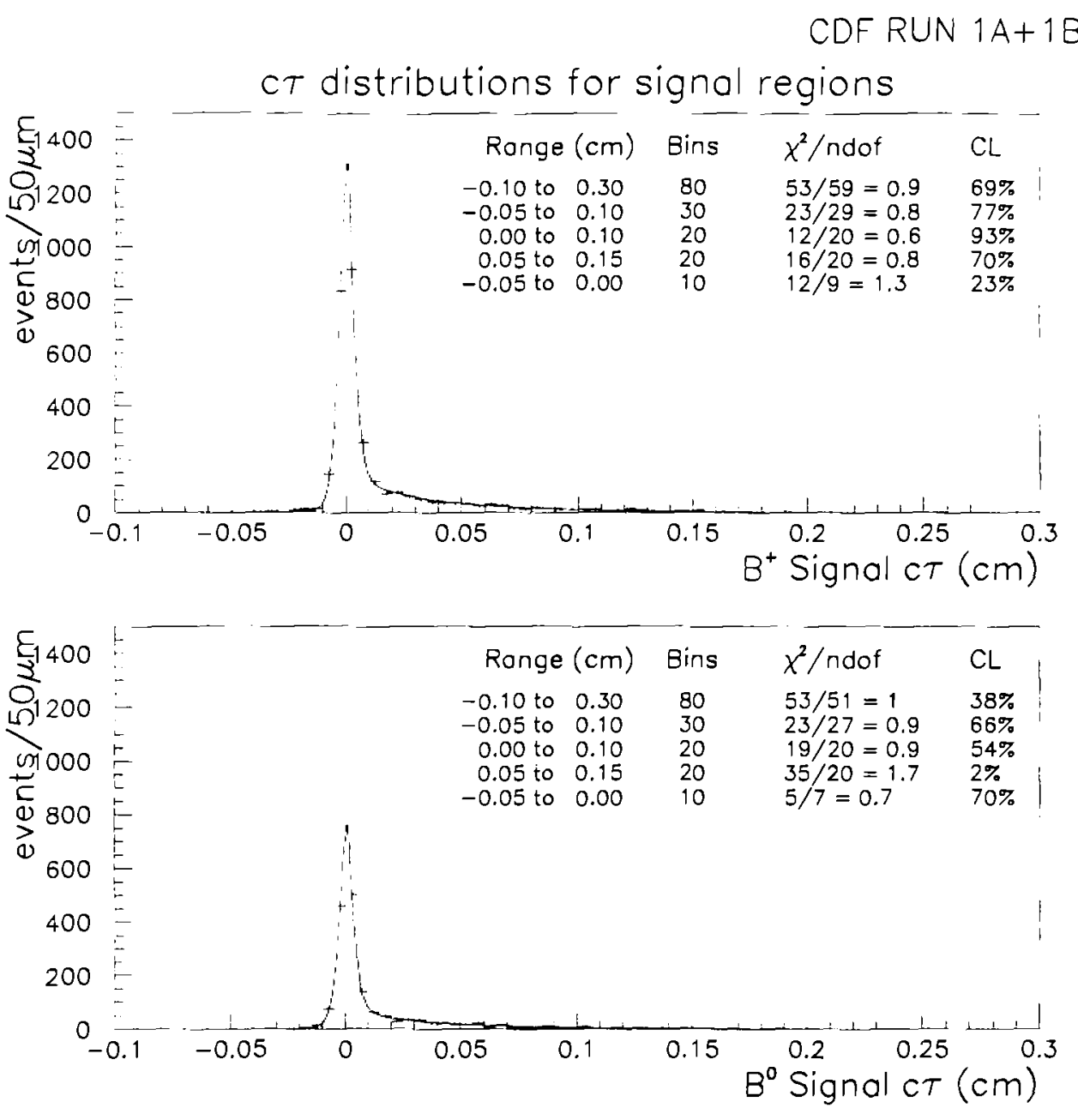

Figure 6.16: Evaluation of the fit to the peak distributions for the modeling of non-Gaussian contributions by $\mathcal{R}$. 

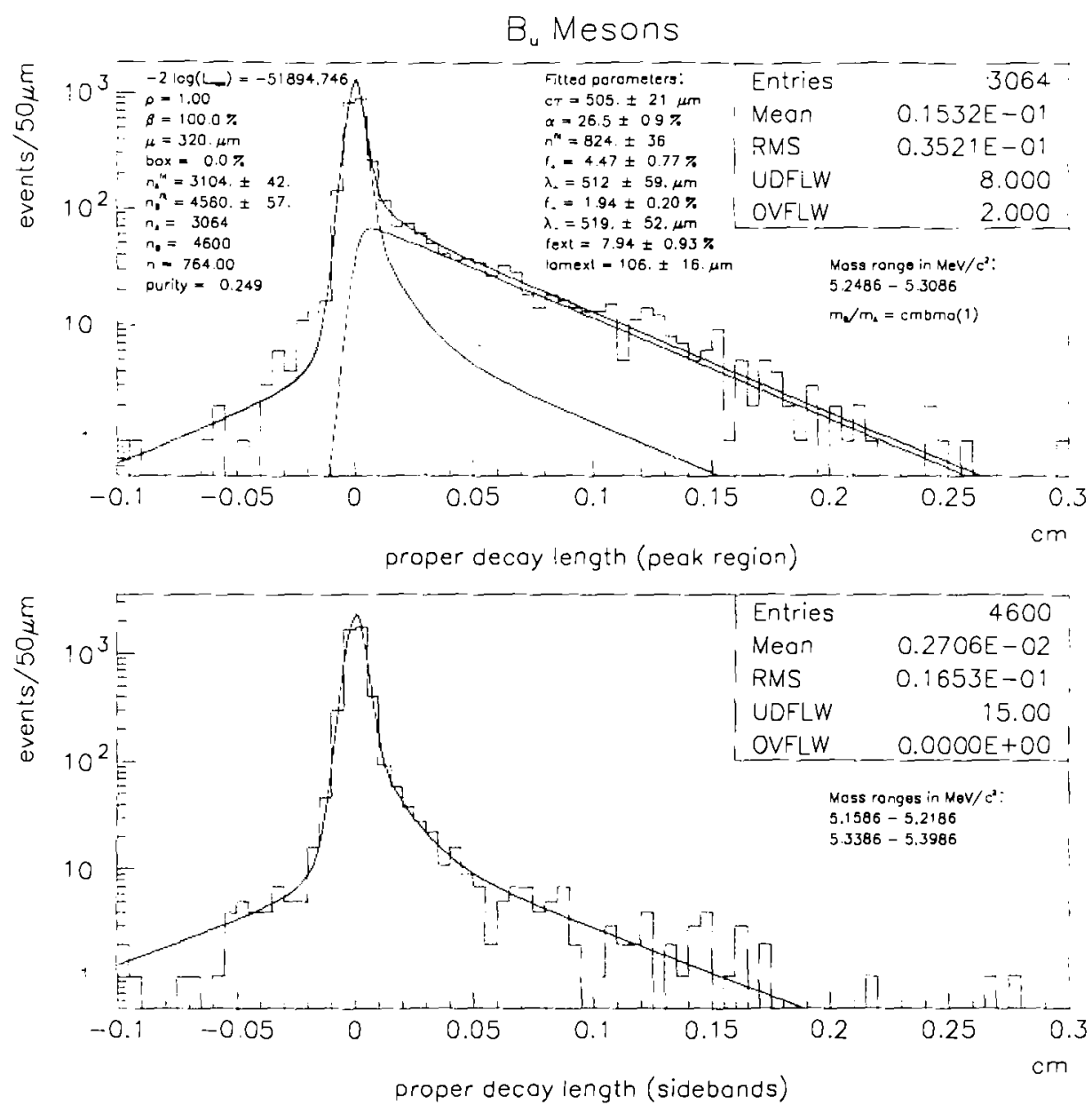

Figure 6.17: Fit to the charged distributions in which all three exponential slopes are free to vary. The magnitude of slopes $\lambda_{+}$and $\lambda_{-}$are virtually the same while the slope lamext is equivalent to $\lambda_{2}$ in the default fit. 

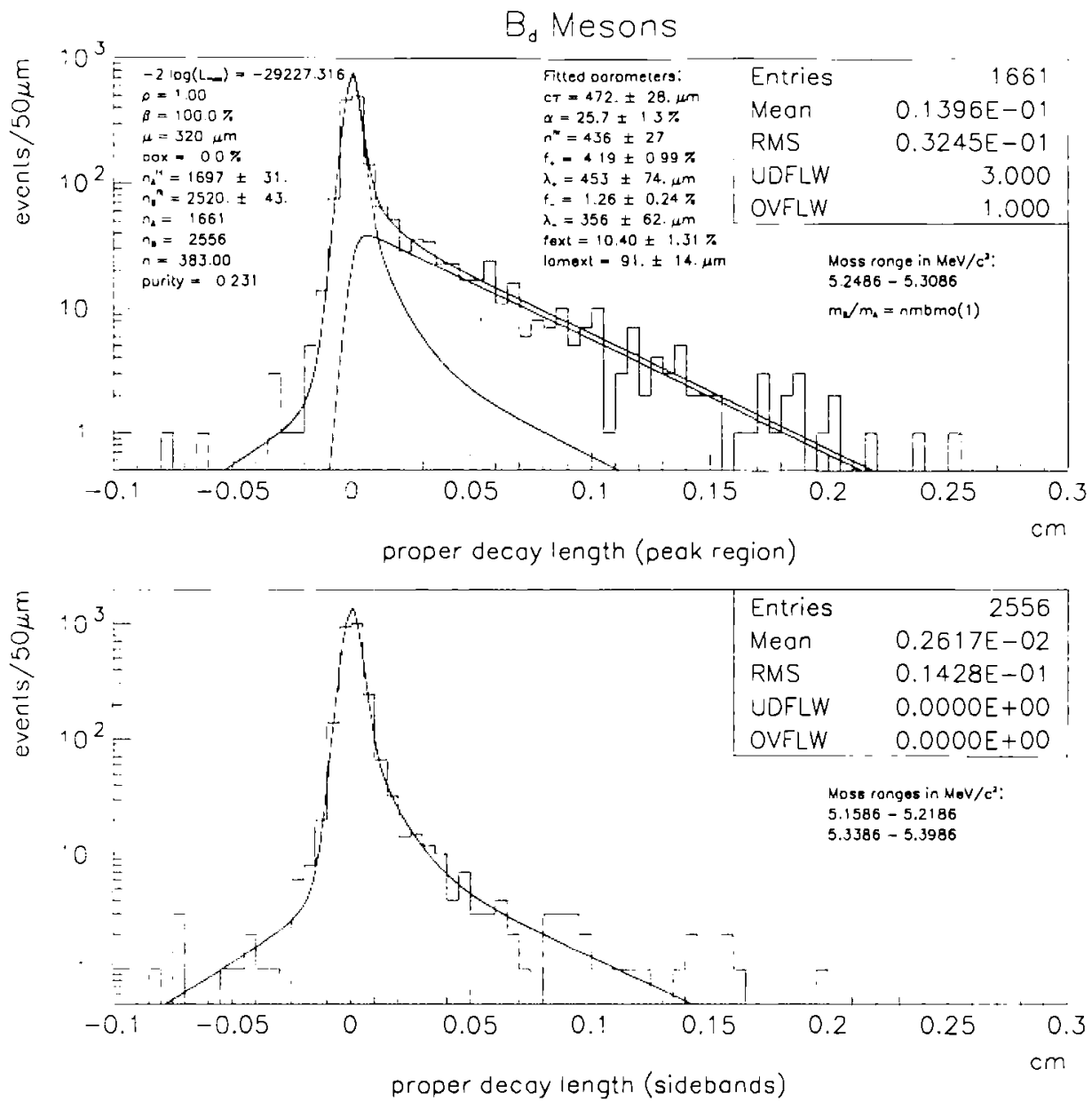

Figure 6.18: Fit to the neutral distributions in which all three exponential slopes are free to vary. 
A fit bias is assigned to the distributions which stems from the Monte Carlo samples described in Chapter 5. Bias due to the fitting procedure is construed as the difference between the mean of the $c \tau$ distribution obtained by the 1000 Monte Carlo generated samples and the measured value for its respective $B$ sector. Table 6.2 lists the systematics included in the exclusive analysis for the $B_{u}$ and $B_{d}$.

In comparing with Run $1 \mathrm{~A}[6.4]$ it is seen that there is no 'box' systematic. The box systematic acted to introduce a flat background component in the range from 0 to $0.2 \mathrm{~cm}$ in $\mathrm{c} \tau$. Its contribution to the probability density function is a simple $f_{\text {box }} e^{-0}$ in the sidebands and background parts. In whatever manner it is described, its ultimate effect is to account for longer lived background and corresponding sideband events seen in the $c \tau$ distributions. Thus, the box systematic is a measure of the fit's inability to describe the background shape, due in large part to those long lived events. With the addition of a second high side exponential in the current fit to account for these long lived sideband events, the box systematic has been removed from the systematics. 


\section{Bibliography}

[6.1] Hans Wenzel and Doug Benjamin, Run 16 inclusive b-lifetime measurement, CDF internal document number 3460 .

[6.2] A store is simply the nomenclature given to a group of runs for a given antiproton injected stack.

[6.3] I wish to give thanks to Rick Snider who graciously offered this plot during the writing of CDFNOTE 3051.

[6.4] Olivier Snider, Alan Spies, et al., A Measurement of $B^{+}$and $B^{\circ}$ Lifetimes Using Exclusive Decay Channels, CDF internal document number 2345 .

[6.5] C. H. Wang, et al., Alignment of SVX' Using Run 1B Data, CDF internal document number 3002 .

[6.6] F. Bedeshi, et al., Status Report on SVX Alignment, CDF internal document number 1948. 


\section{Chapter 7}

\section{Final Results}

With the completion of the systematic error analysis, the lifetimes for the $B_{u}$ and $B_{d}$ are listed separately as well as the ratio of the two in this section. The $B$, lifetime is also quoted with statistical error only and the result is compared with a previously approved result released by F. Azfar on behalf of the CDF collaboration.

\subsection{B Lifetimes}

The results of the analysis yield

$$
\begin{gathered}
\tau_{B_{u}}=1.68 \pm 0.07(\text { stat }) \pm 0.02(\text { sys }) p s \\
\tau_{B_{d}}=1.58 \pm 0.09(\text { stat }) \pm 0.02(\text { sys }) p s \\
\tau_{B_{u}} / \tau_{B_{d}}=1.06 \pm 0.07(\text { stat }) \pm 0.01(\text { sys }) .
\end{gathered}
$$

The length scale error is completely correlated across the two sectors since they apply to the SVX radial shifts which are the same regardless of B sector. The lifetime ratio agrees with HQET predictions which state that the ratio should be close to 1 . Each sector is further examined by determining the proper decay length and number of fitted events for $b$ versus $\bar{b}$ distributions. 
These numbers are given in Table 7.1 for $B^{+}$versus $B^{-}$and $B^{\circ}$ versus $\overline{B^{\circ}}$. For this analysis it is only possible to discern $B^{\circ}$ from $\overline{B^{\circ}}$ by linking the kaon charge from the $\mathrm{K}^{*}(892)^{\circ}$ back to the $b$ or $\bar{b}$. The results show no significant difference in the lifetimes.

\begin{tabular}{|c|c|c|}
\hline \hline & $\mathrm{c} \tau$ & $n_{\text {fit }}$ \\
\hline$B^{+}$ & $504 \pm 29 \mu \mathrm{m}$ & $435 \pm 26$ \\
$B^{-}$ & $506 \pm 31 \mu \mathrm{m}$ & $388 \pm 26$ \\
$B^{\circ}$ & $522 \pm 43 \mu \mathrm{m}$ & $190 \pm 17$ \\
$\overline{B^{\circ}}$ & $449 \pm 45 \mu \mathrm{m}$ & $171 \pm 18$ \\
\hline
\end{tabular}

Table 7.1: $b$ versus $\bar{b}$ comparison in which the errors are statistical.

The $B_{u}$ and $B_{d}$ exclusive lifetimes are consistent with those quoted for the semileptonic modes, $B \rightarrow l D X$, in which the values quoted for Run $1 \mathrm{~A}$ are[7.1]:

$$
\begin{gathered}
\tau_{B_{u}}=1.56 \pm 0.13(\text { stat }) \pm 0.06(\text { sys }) p s \\
\tau_{B_{d}}=1.54 \pm 0.08(\text { stat }) \pm 0.06(\text { sys }) p s \\
\tau_{B_{u}} / \tau_{B_{d}}=1.01 \pm 0.11(\text { stat }) \pm 0.02(\text { sys }) .
\end{gathered}
$$

The two lifetimes have also been investigated by the ALEPH collaboration[7.2] in which they find

$$
\begin{gathered}
\tau_{B_{u}}=1.58 \pm 0.06(\text { stat }) \pm 0.03(\text { sys }) p s \\
\tau_{B_{d}}=1.55 \pm 0.06(\text { stat }) \pm 0.03(\text { sys }) p s \\
\tau_{B_{u}} / \tau_{B_{d}}=1.03 \pm 0.08(\text { stat }) \pm 0.02(\text { sys }) .
\end{gathered}
$$

They use a combined sample of partially reconstructed decays such as $B \rightarrow$ $l D X$, and exclusive decay modes from $B \rightarrow \Psi K$. The CDF results from the semileptonic and the exclusive modes have been combined to give a CDF average of

$$
\tau_{B_{u}}=1.66 \pm 0.07 p s
$$




$$
\begin{gathered}
\tau_{B_{d}}=1.56 \pm 0.07 p s \\
\tau_{B_{u}} / \tau_{B_{d}}=1.05 \pm 0.06
\end{gathered}
$$

in which the error is the sum of statistical and systematic errors. Error correlations among the $\mathrm{B}$ sectors are considered when evaluating the errors.

The results for the $B_{\text {o }}^{\circ}$ lifetime yield,

$$
\tau_{B_{i}^{\circ}}=1.28 \pm 0.21(\text { stat }) p s
$$

for $73 \pm 12$ signal events. The strange $B$ meson lifetime is in good agreement with that approved by the CDF collaboration, in which F. Azfar[7.3] found a value of

$$
\tau_{B_{g}}=1.34_{-0.19}^{+0.23}(\text { stat }) \pm 0.05(\text { sys }) p s
$$

for $58 \pm 8$ signal events. 


\section{Bibliography}

[7.1] F. Abe, et al., Measurement of the $B^{-}$and $\bar{B}^{\circ}$ Meson Lifetimes Using Semileptonic Decays, Phys Rev Lett. 76 (1996) 4462.

[7.2] The ALEPH Collaboration, Improved Measurement of the $\bar{B}^{\circ}$ and $B^{-}$ Meson Lifetimes, CERN-PPE/96-14.

[7.3] F. Azfar, J. G. Heinrich, and N. S. Lockyer, An Update on the Measurement of the $B_{s}^{\circ}$ Lifetime from the Decay $B_{a}^{\circ} \rightarrow J / \psi \phi$. CDF internal document number 3423 . 


\section{Vita}

The author was born in Lancaster, PA on August 9, 1968. After attending Manheim Township High School the author completed a mathematics and physics undergraduate program at Franklin and Marshall College while participating in its varsity football program as well as club volleyball. A recipient of numerous undergraduate awards, he turned down a chance to play semiprofessional football in order to attend graduate school in physics at The Johns Hopkins University. During completion of his Master of Arts degree, the author was a member and coach of the men's club volleyball team in which they went undefeated in 1992. The author went on to complete a doctoral thesis while stationed at Fermilab for research on the lifetimes of charged and neutral $B$ mesons. 